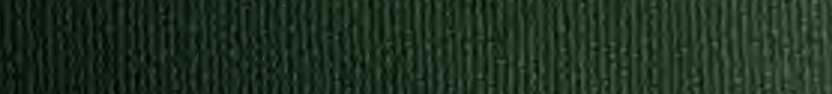
(26)

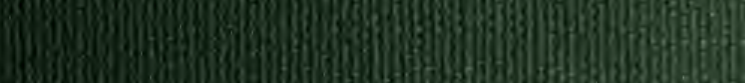
ank

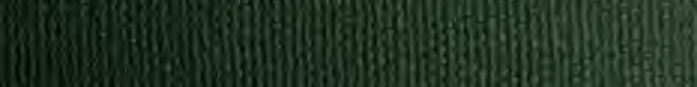
 W.

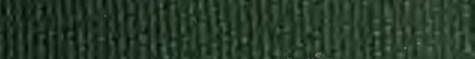

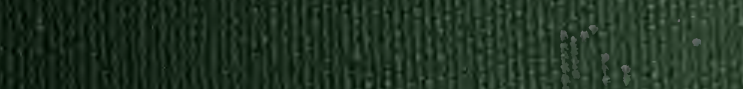

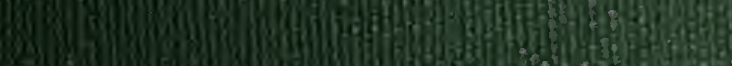

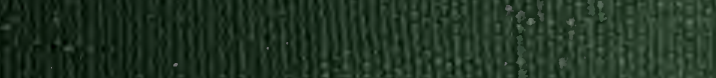
XY)

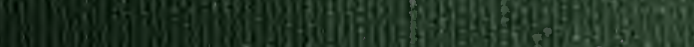

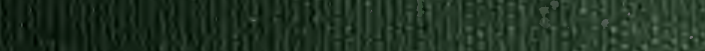

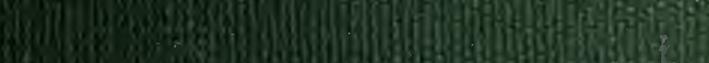
W. Why 6) 19.

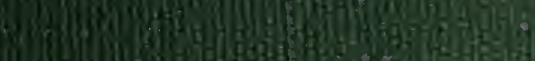
U.

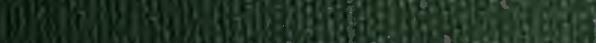

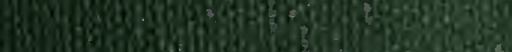
Uhm

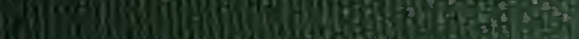
Kondy W. aWM

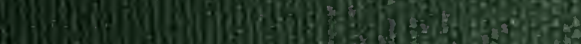

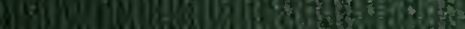
3. 6. W.

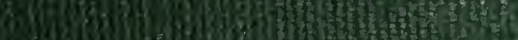
6.5.

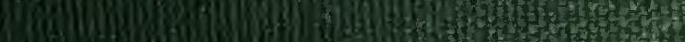
165.7.

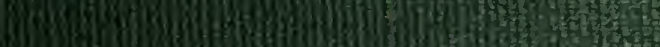
10.13.

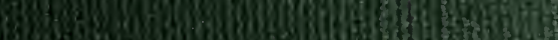

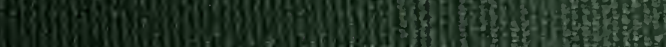

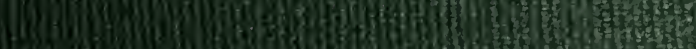

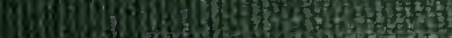
I. Mulf

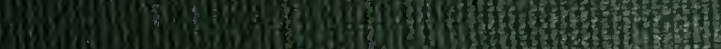

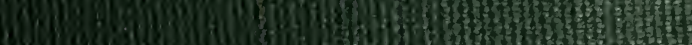
(1)

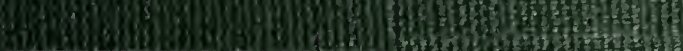




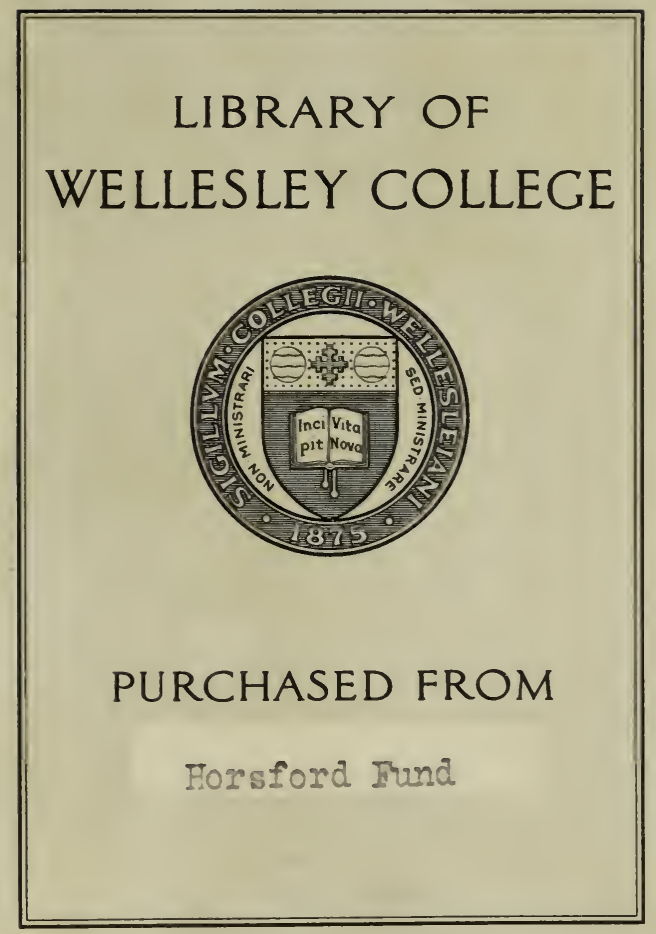






\title{
Manual of The Odonata of New England
} by R. Heber Howe, Jr.

\author{
PART I: March, 1917, pages 1-8 \\ PART II : August, 1917, pages 9-24 \\ PART III: August 30, 1918, pages 25-40 \\ PART IV : March 25, 1919, pages 41-66 \\ PART V: January 8, 1920, pages 67-94 \\ PART VI: March 15, 1920, pages 95-102
}

\author{
Memoir of \\ the Thoreau Museum of Natural History: II \\ Middlesex School \\ Concord, Massachusetts
}





\section{MEMOIR}

OF THE

\section{THOREAU MUSEUII OF NATURAL HISTORY: II}

\section{Manual of the Odonata of New England}

By R. HEBER HOWE, Jr.

\section{PART I.}

Mareh, 1917.

In 1905 Dr. P. P. Calvert published * a list of the Odonata of New England. Since that date a considerable number of species have been added to his list, bringing the number known from this area to over one-hundred and fifty. Our distributional knowledge of species in the six states of this group has also been very much enlarged.

The present work is an attempt to supply a field manual of the New England Odonata for the use of all classes of students. A pictorial key of genera, and illustrations of the diagnostic characters of species are given, in the hope that an easy recognition of these insects will lead to a more general study of them. The habitat, abundance, limital dates of capture, and all known stations are recorded, and for the most part the ranges mapped.

The classification as given by Dr. Muttkowski in his Catalogue of the Odontata of North America (1910) has been adopted. The author wishes to espress his sincere thanks to Mr. E. B. Williamson, Dr. R. A. Muttkowski, Mr. L. B. Woodruff, Dr. E. M. Walker, Mr. C. W. Johnson, Dr. Samuel Henshaw, Mr. Nathan Banks, Dr. R. P. Currie; Dr. F. E. Lutz, Dr. P. P. Calvert, Messrs. D. M. and B. K. Little, Mr. W. T. Davis, Mr. D. L. Dutton, Mr. A. P. Morse, and to my daughter, Miss S. A. Howe, for valued help.

\section{Order: Odonata Fabricius}

* No. 6. Occasional Papers of the Boston Soc. Nat. Hist. 7: Fauna of N. E., Oct. 1905 .

SCIENCE

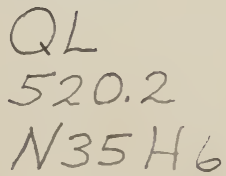


Pictorial Key of Genera

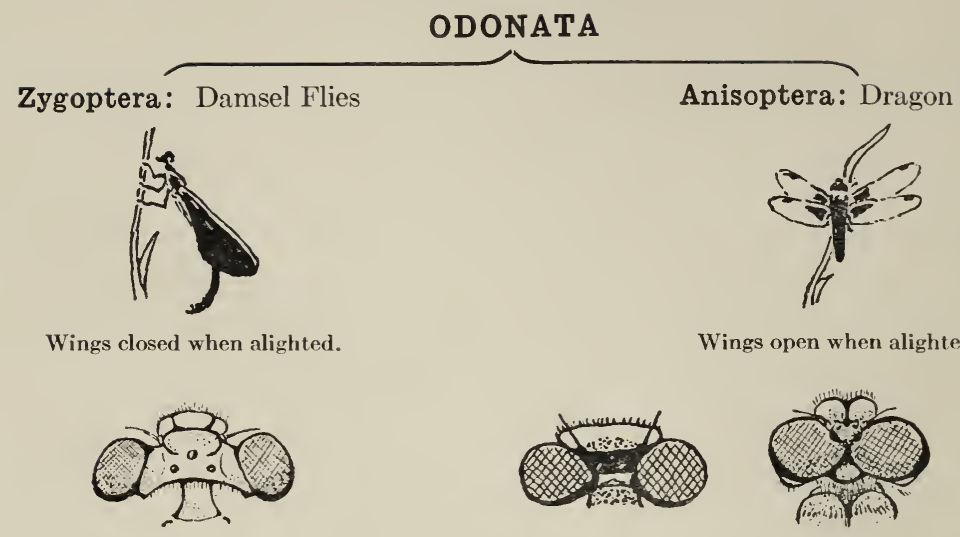

Eyes widely separated, by more than the full width of an eye.

Eyes approximate, separated by less than half the width of an eye. (See Aeshnidae and Libellulidae).

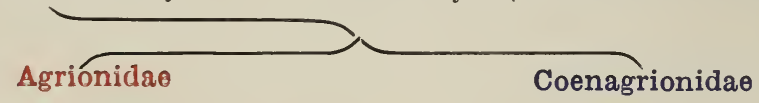

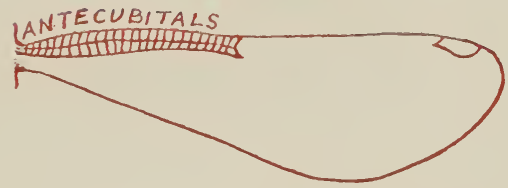

Five or more antecubitals. Wings not stalked.

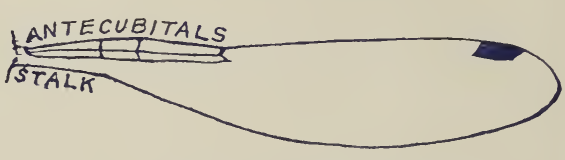

Two (rarely three) antecubitals.

Wings stalked (see page 6).

Agrioninae

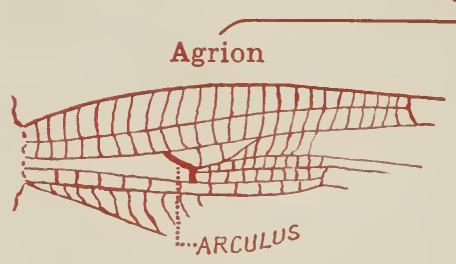

Arculus bent.

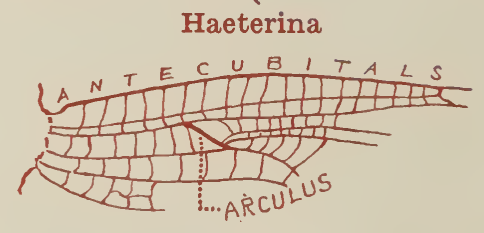

A reulus straight.

Suborder: Zygoptera Selys

Family: Agrionidae Leach

Subfamily: Agrioninae Kirby

Genus: Agrion Fabricius

Agrion amatum

dimitiatum apicale

aequabile

maculatum
Me. N. H. Vt. Mass. R. I. Conn.

$$
\begin{aligned}
& +\quad+ \\
& ++++ \\
& +++++
\end{aligned}
$$


a. I' ings narrow (7-9 $\mathrm{mm}$.),

front and hind margins nearly parallel.

Pterostignia in all females occasionally absent or poorly developed.

1. Agrion amatum (Hagen).

Psyche 5: 244. 1889. Type locality: "Dublin, New Hampshire."

Rare,- cold, wooded, mountain brooks, alt. 500 to $1300 \mathrm{ft}$.

June 18 to July 14.

New Hampshire: Franconia (Slosson); Dublin (Hagen); Peterboro (Cabot); Intervale (U. S. N. M.).

Massachusetts: Montgomery (M. C. Z. \& U.S. N. M.).

Connecticut: Litchfield (Woodruff).*

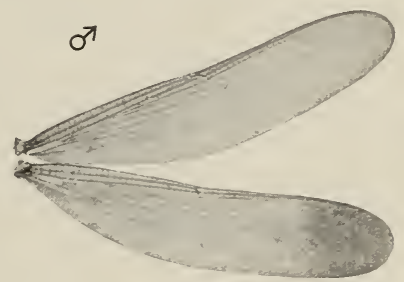

\section{Agrion dimidiatum apicale} (Burm).

Handb. ent. 2: 827. 1839. Type locality: "Vaterland unbekannt." (Apparently Philadelphia).

Very rare,-brooks and ditches. This species is often confused with the following, and all re-

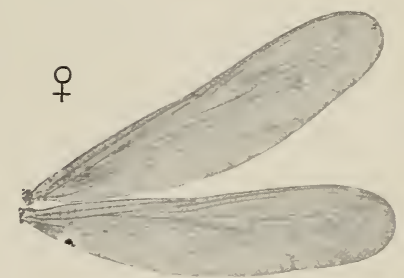

A. amatum (natural size).

Wings fuliginous, hind wings $\sigma^{7}$ cords need careful verification.

June 21 to July 21.

Massachusetts: Waltham, South Reading (Hagen); Carver (B. S. N. H.); Medfield (Morse).

b. Wings wide (9-10 $\mathrm{mm}$.), hind margin bowed.

\section{Agrion aequabile (Say).}

Journ. acad. nat. sci. Phila. 8: 33. 1839. Type locality: "Massachusetts."

Common, - brooks, creeks, and ditches. May to July 21.

* Jour. N. Y. Ent. Soc. 22: 155. 1914.

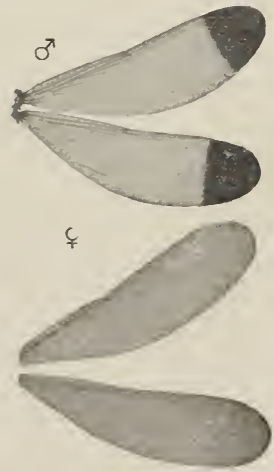

A. d. apicale (natural size). Wings hyaline or fuliginous; $\sigma^{\top}$ brackish at tips. 
Maine: Foxcroft, South Lagrange, Orono, Bradley, Auburn (Harvey); Manchester (Wadsworth); Bethel, Norway (Hagen); Machias (B. S. N. H.).

New Hampshire: Peterboro (M. C. Z.); Piscataqua river (M. C. Z.).

Vermont: Newport (Slosson).

Massachusetts: Worcester (Hitchings); Sherborn (Babcock); Lawrence, Brookline, Tyngsboro (Hagen); North Reading, Great Barrington, North Adams, (B. S. N. H.); Concord (Howe); South Natick (Morse); Norfolk Co., (M. C. Z.).

Connecticut: Litchfield (Woodruff).

\section{Agrion maculatum Beaur.}

Ins. Afr. Amer. 85. pl. 7. f. 3. 1805. Type Locality: "Etat-Unis d'Amerique."

Abundant, - woodland and grass grown brooks, and streams.

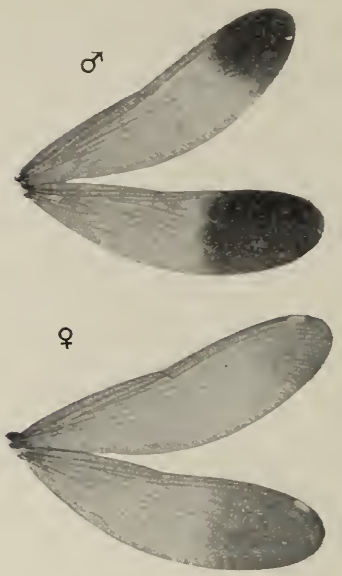

A. aequabile (natural size). Wings fuliginous; blackish or or brown of at tips.

April 16 to August 27.

Maine: No. East Carry, Foxcroft, South Lagrange, Orono, Bradley, Farmington (Harvey); Manchester (Wadsworth); Norway (Hagen); Mt. Desert (Bullock); Wales, Machias (B. S. N. H.); Noreross (U. S. N. M.).

New Hampshire: Meredith Neck (Howe); Franconia (U. S. N. M.).

Vermont: Newport (Slosson); Bennington (B. S. N. H.); Brandon (Dutton).

Massachusetts: Ipswich, Cambridge, Milton, Sutton, Northampton (Hagen); Sherborn (Babcock); Nahant, Dorchester, Andover, Tyngsboro, Brookline (M. C. Z.); Hopkinton, Blue Hills, Auburndale (B. S. N. H.); North Saugus (U.S. N. M.); Concord (Howe).

Rhode Island: Apponaug (B. S. N. H.).

Connecticut: Litchfield (Woodruff); Darien, Rowayton (B. S. N. H.); Devil's Garden (U. S. N. M.).

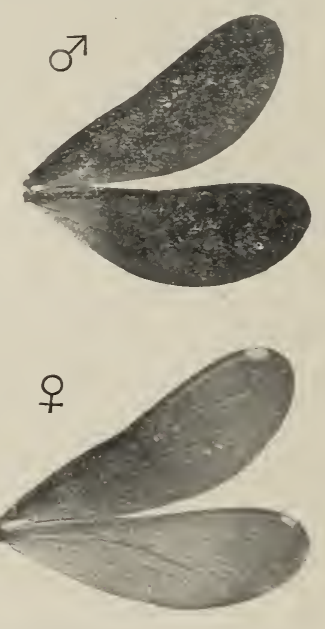

A. maculatum (natural size). Wings black $\sigma^{7}$, and brown ? 


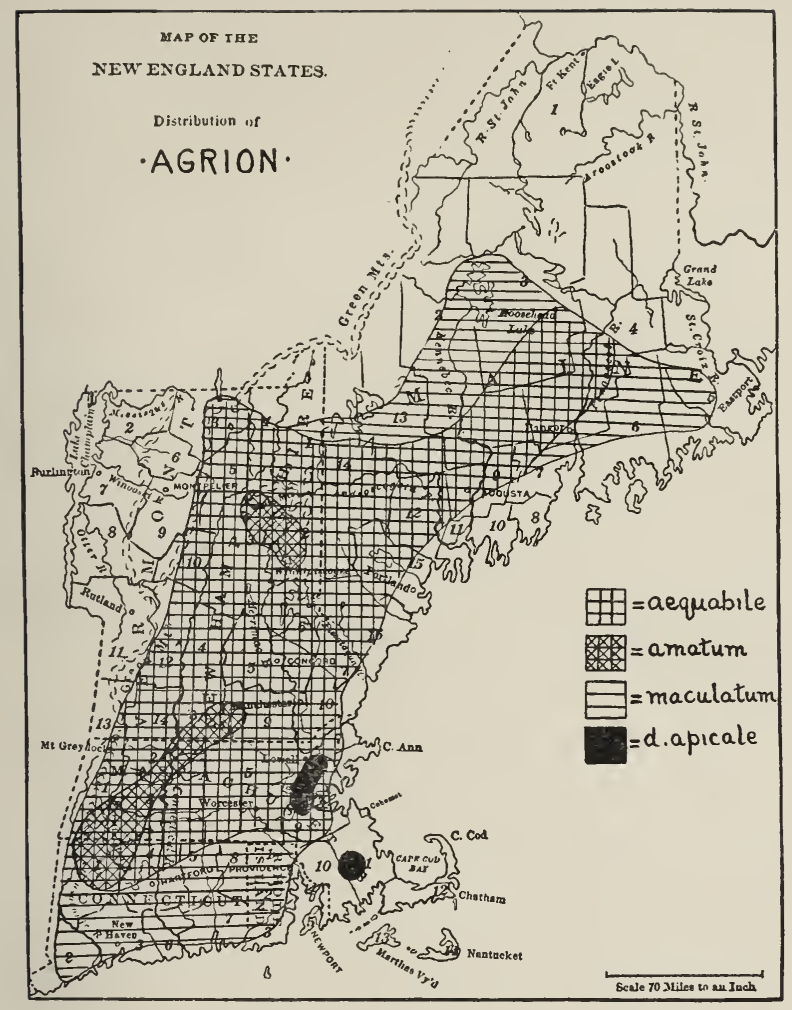

Genus: Haeterina Hagen

Me. N. H. Vt. Mass. R. I. Conn.

Haeterina americana

$+\quad+$

5. Haeterina americana (Fabr.).

Ent. syst. suppl. 287. 1798. Type locality: "America."

Uncommon, - meadow brooks, ditches, and rivers.

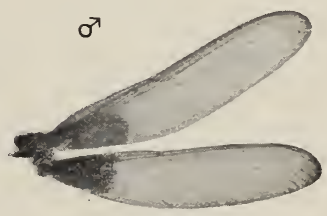


June 22 to September 16 .

Maine: Chemo Mills, Bradley, Winslow, Auburn (Harvey); Norway (Hagen).

Massachusetts: Salem, IVeston (Hagen);

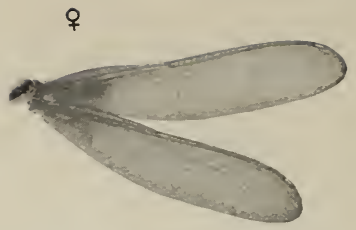

Wellesley (B.S. N. H.); Sherborn (Morse);

Cambridge, Provincetown (M. C. Z.).

H. americana (natural size).

Wings yellowish; red $\sigma^{\top}$ or orange $\&$ at base.

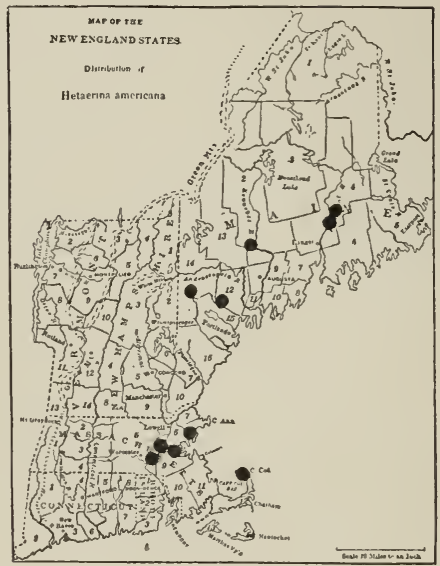

Note: B.S. N. H.= Boston Society of Natural History, Boston, Mass.; U.S. N. M.= U.S. National Museum, Washington, D.C.; M.C.Z. = Museum Comparative Zoölogy, Cambridge, Mass.

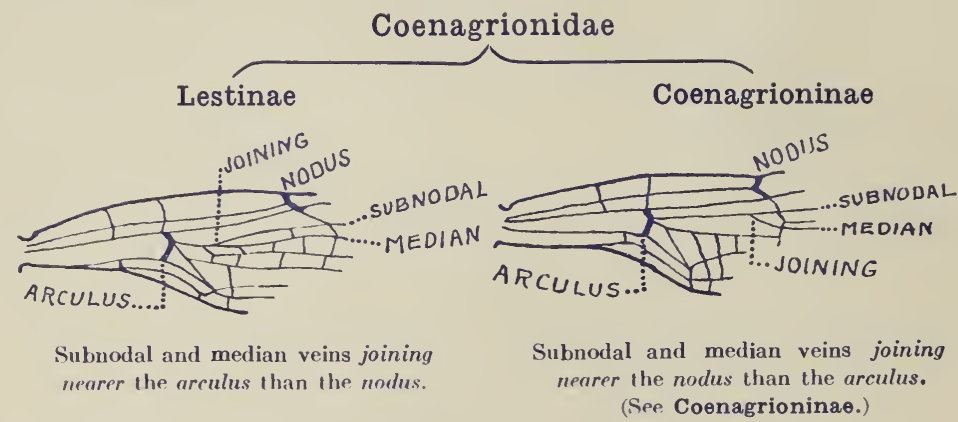

Family: Coenagrionidae Kirby Subfamily: Lestinae Needham

Genus: Lestes Leach 
Lester eurinus

congener

unguiculatus

uncatus

disjunctus

forcipatus

rectangularis

vigilax

inequalis

Me. N.H. Vt. Mass. R.I. Conn.

A. Wings $\sigma^{7}$ distinctly fuliginous. $\sigma^{\pi}$ wings $25-29$

$\mathrm{mm}$. long. क्ष wings 27-28 $\mathrm{mm}$. long.

NotE: The females of all agrionines are difficult to determine as they have few marked characters, and are of ten dimorphic. Pairs taken in copulation will supply material for study.

The drawings of the male abdominal appendages are from Dr. Calvert's Catalogue of the Odonata of Philadelphia (Trans. Amer. Ent. Soc. 20: 152-272, 1893), and are republished with permission.

\section{Lestes eurinus Say.}

Jour. acad. nat. sci. Phila. 8 :36. 1839. Type locality: "Massachusetts."

Uncommon,- - ponds.

July 10 to 20 .

Massachusetts: Sherborn (Babcock); Milton (Hagen); Woods Hole (Kellicott); Brookline, Province-

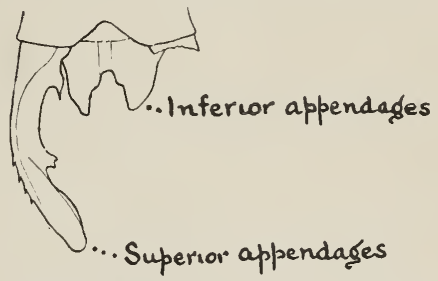
town (M. C. Z.); Wellesley, Green Lodge (Morse); Concord (Howe).

Connecticut: Burchard's Pond, Fairfield Co. (U. S. N. M.).

B. Wings $\sigma^{\top}$ and o hyaline. ơ wings less than $25 \mathrm{~mm}$., o wings less than $24 \mathrm{~mm}$. long.

a. $\sigma^{7}$ inferior appendages less than half as long as superior appendages. $\quad$ 's with a yellow mid-dorsal line along the abdomen.

\section{Lestes congener Hagen.}

Syn. Neur. N. Amer. 67. 1861. Type locality: "New York."

Uncommon,-- ponds and ditches.

July 29 to October 9 .

Maine: North West Carry, Orono (Harvey); Manchester (Wadsworth).

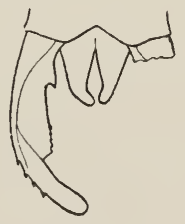


New Hampshire: Franconia (U. S. N. M.).

Massachusetts: Concord (Howe); West Chop (Morse).

Connecticut: Litchfield (Woodruff).

b. $\sigma^{7}$ inferior appendages over half as long, but not longer than the superior appendages. $\quad$ 's with a yellow line across the back of the head.

\section{Lestes unguiculatus Hagen.}

Syn. Neur. N. Amer. 70. 1861. Type locality: "Chicago; Bergen Hill, New Jersey; New York; St. Louis; Wisconsin."

Common,- ponds and ditches.

June 17 to September 5.

Maine: North West Carry, Orono (Harvey); Manchester (Wadsworth); York Harbor (Moore).

Vermont: Brandon (Dutton).

Massachusetts: Worcester (Hitching); Cuttyhunk island (Calvert); Martha's Vineyard (Moore); Concord (Howe); Provincetown, Melrose Highlands (U. S. N. M.).

Rhode Island: Providence (Davis); Block Island (Woodruff).

1. $\sigma^{\pi}$ inferior appendages straight, or nearly so. $\sigma^{7}$ wings less than $22 \mathrm{~mm}$. long. $\quad$ o with no yellow line on back of head.

Wings less than $24 \mathrm{~mm}$. long.

\section{Lestes uncatus Kirby.}

Cat. Odon. 160, 1890. Type locality:

"Chicago; Washington; Wisconsin."

Uncommon,- ponds and ditches.

June 16 to September.

Maine: Orono (Harvey); Manchester (Wadsworth).

New Hampshire: Franconia (Slosson); North Conway (Calvert); Squam lake (B. S. N. H.).

Vermont: Brandon (Dutton).
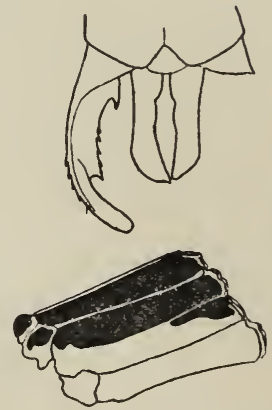

o Side thoracic markings: light line (humeral) between dark bands uniformly narrow.

Massachusetts: Cambridge, Milton (Hagen); Worcester (Hitchings); Concord (Howe); Mt. Everett, Chester (B. S. N. H.).

Connecticut: Darien (B. S. N. H.).

(To be continued.) 
Memoir of the Thorean Musemm of Natural History: II

\section{Manual of the Odonata of New England}

By R. HEBER HOWE, Jr.

\section{PART II. \\ August, 1917.}

\section{Lestes disjunctus Selys.}

Bull. acad. Belg. 16: 210. 1862. Type locality: "Nouvelle-Ecosse, Maine, Illinois, Chicago."

Uncommon,-ponds, ditches, and brooks. June to September 18.

Maine: Orono, Fryeburg (Harvey); Manchester (Wadsworth); Norway (M. C. Z.).

New Hampshire: Fabyan's (Calvert); Franconia (Slosson); Moultonboro, Meredith Neck (Howe).

Massachusetts: Concord, Nantucket (Howe); Norfolk Co. (M. C. Z.); Provincetown (B. S. N. H.).

Connecticut: Litchfield (Woodruff); North

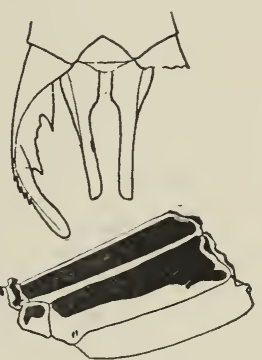

o light line narrow, but widening slightly anteriorly. Windham, South Kent (Morse).

\section{Lestes forcipatus Rambur.}

Ins. Neur. 246. 1842. Type locality: "l'Amérique septentrionale."

Not uncommon,- ponds and ditches.

June 27 to September 5 .

Maine: Orono, Fryeburg (Harvey); Manchester (Wadsworth).

Massachusetts: Sherborn (Babcock); Worcester (Hitchings); Cuttyhunk Island (Calvert); Woods Hole (U. S. N. M.); Concord, Monument Beach, (Howe).

Connecticut: New Haven (Moore); Litchfield (Woodruff).

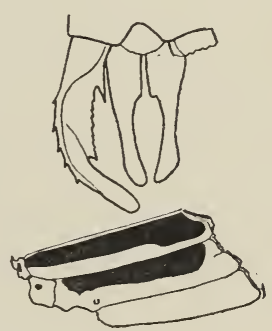

o light line uniformly wide $\frac{3}{4}$ its length.

\section{Lestes rectangularis Say.}

Journ. acad. nat. sci. Phila. 8: 34. 1839. Type locality: "Indiana, Massachusetts." 
Common,- ponds, brooks, and ditches. June 21 to September 19.

Maine: Orono, Fryeburg (Harvey); Manchester (Wadsworth).

New Hampshire: Franconia (Slosson); Moultonboro, Meredith Neck (Howe).

Massachusetts: Milton (Hagen); Worcester (Hitchings); Cuttyhunk Island (Calvert); Sherborn (B. S. N. H.); Medfield, Dover (Morse); Winchendon, Cambridge, Saugus, Brookline,

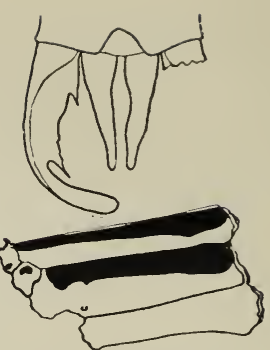

ㅇ light line widening anteriorly.

Norfolk Co. (M. C. Z.); Woods Hole, Provincetown (U. S. N. M.); Concord, Monument Beach (Howe); Hingham (Barnes).

Rhode Island: Providence (Davis); Block Island (Calvert); Bristol (Howe).

Connecticut: Litchfield (Woodruff); Bethlehem (U. S. N. M.).

2. $\sigma^{7}$ wings more than $22 \mathrm{~mm}$. long. \& wings more than $24 \mathrm{~mm}$. long.

\section{Lestes vigilax Hagen.}

Bull. acad. Belg. 16: 214. 1862. Type locality: "New-Jersey."

Common,- - lakes, ponds, and ditches.

June 19 to September 5 .

Maine: Manchester (Wadsworth); Fryeburg (Harvey); Cape Elizabeth (M. C. Z.); Orono (U. S. N. M.).

New Hampshire: Moultonboro, Meredith Neck (Howe).

Massachusetts: Worcester (Hitchings); Nantucket (Albertson); Concord, Wareham (Howe); Medfield, Sherborn, (Morse); Natick, Cambridge (M. C. Z.). vert).

Rhode Island: Block Island, Meredith Neck (Cal-

Connecticut: Litchfield (Woodruff); South Kent (Morse); Darien (U. S. N. M.).

c. $\sigma^{7}$ inferior appendages longer than superior. $q$ back of head entirely yellow.

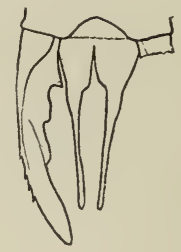




\section{Lestes inequalis Walsh.}

Proc. acad. nat. sci. Phila. 385. 1862. Type locality: [Rock Island, Illinois.]

Rare,- - lakes, ponds, and ditches.

\section{June 17 to August 15.}

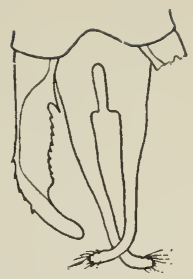

Maine: Bradley, Orono (Harvey); Manchester (Wadsworth).

New Hampshire: Franconia (Slosson); Moultonboro, Meredith Neck (Howe).

Massachusetts: Cohasset, Blue Hills, North Reading, Manomet (B. S. N. H.); Hyde Park (M. C. Z.); Sherborn (Morse).

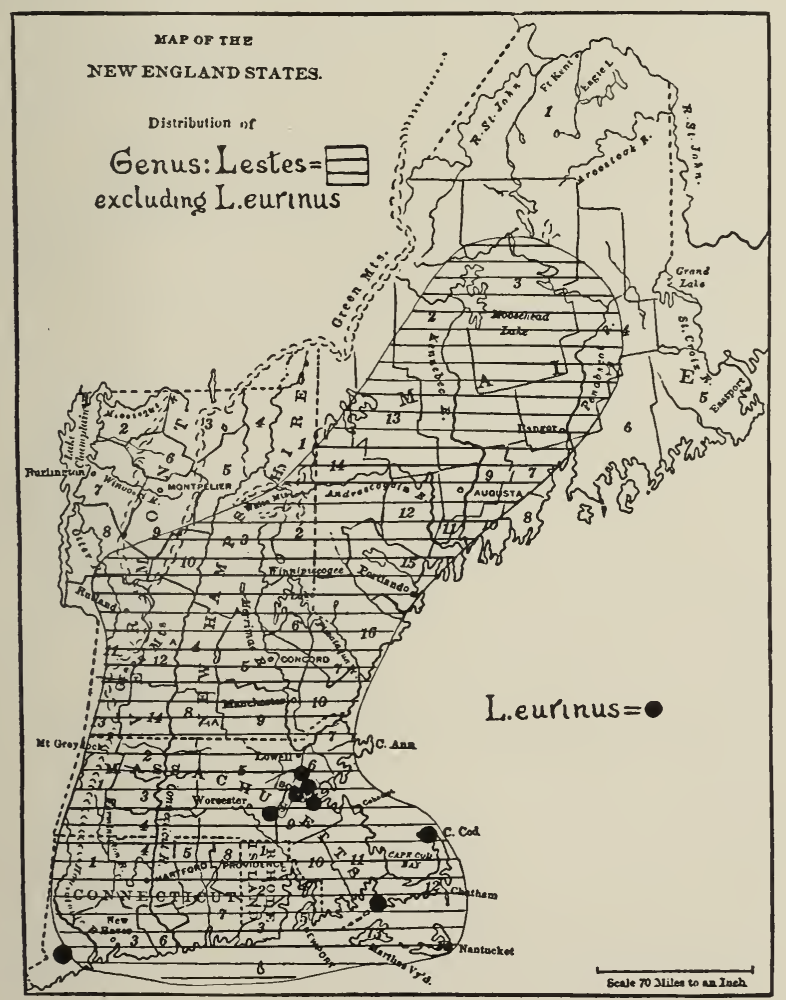

Note: Since the publication of Part I, I have secured a record of $L$. unguiculatus for New Hampshire: White Mts., (M. C.Z.). I have also found the species common at Nantucket, Hingham (Barnes), Cohasset (Warden), and Woods Hole, Mass., and Middletown, R. I. 


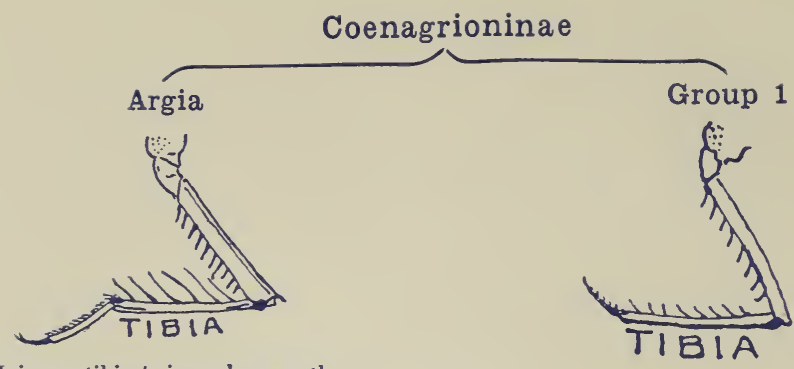

Hairs on tibia twice as long as the spaces between the hairs.

Hairs on tibia never twice as long as the spaces between the hairs (see Group 1).

Subfamily: Coenagrioninae Kirby

Genus: Argia Rambur

Argia moesta

Argia violacea

Argia sedula

Me. N. H. Vt. Mass. R. I. Conn.

A. More than one cell under pterostigma.

15. Argia moesta (Hagen).*

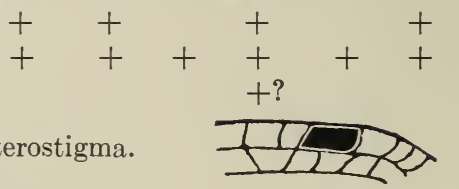

Syn. Neur. N. Amer. 94. 1861. Type locality: "Pecos River, Western Texas."

*=A. moesta var. putrida (Hagen) see Ent. News 23: 196-203, 1912.

Common, - ponds and lakes.

June 25 to August 30.

Maine: Orono (Harvey); Manchester (Wadsworth);

Liberty, Pudding pond (B. S. N. H.); Bradley (U. S. ơ Side view last N. M.).

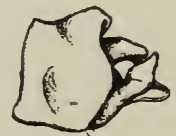

New Hampshire: Moultonboro, Meredith Neck, (Howe).

Massachusetts: Worcester (Hitchings); Natick, Sherborn (Morse); Concord (Howe).

Connecticut: Litchfield (Woodruff); Darien (U. S. N. M.).

B. Only one cell under pterostigma of wing .

16. Argia violacea (Hagen).

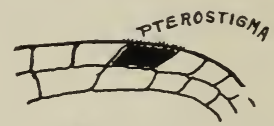

Syn. Neur. N. Amer. 90. 1861. Type locality: "Maryland," etc.

Abundant, - ponds, and lakes.

June 6 to September 10.

Maine: Bradley, Orono (Harvey); Manchester (Wadsworth); MIt. Desert (Bullock). 
New Hampshire: Meredith Neck (Howe).

Vermont: Brandon(Dutton).

Massachusetts: Wareham, Concord (Howe); Medfield, ot Abdominal apDover (Morse); Gloucester (U. S. N. M.).; Hingham (Barnes).

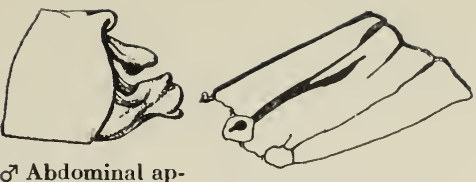
pendages (side view).
\% Side view of thorax.

Rhode Island: Providence (Davis).

Connecticut: West Hartford, Milton, Litchfield (Woodruff).

Noте: Argia apicalis (Say). Though included in Dr. Calvert's list, the record was expunged by Mr. E. B. Williamson (Ent. News 17: 31. 1906).

Argia sedula (Hagen). Two teneral specimens were taken in Concord, Mass., on June 14, 1916, which were doubtfully referred here by Mr. E. B. Williamson.

The figures of the male abdominal appendages are reproduced with permission from Hagen's paper (Bull. Mus. Comp. Zoöl. 34: 1902).

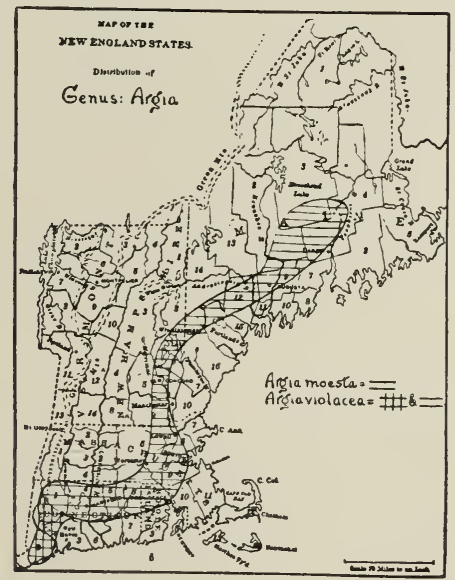

Note: A specimen of $A$. violacea taken since this map was made extends the range to Buzzards Bay.

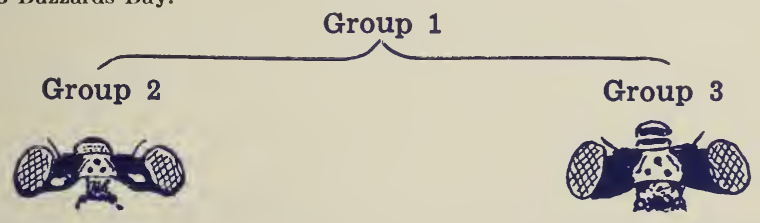

Pale spots (rarely connected by pale narrow line) on top of head.
No pale spots on top of head. (set (roup 4-Chromagrion, Nehalennia, and Amphiagrion.)

\section{Enallagma}

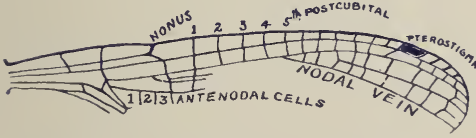

Nodal vein of fore-wing arising near or beyond 5th postecubital.

\section{Group 5}

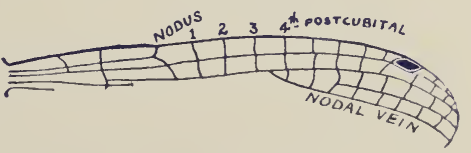

Nodal vein of fore-wing arising not beyond 4th postecubital. (see Group 5-Ischnura and Anomalagrion.) 
Note: The females of this, and the following genera have so few satisfactory characters for determination that specimens taken in copulation will supply the only reliable material for study and comparison. The drawings of the male abdominal segments are in many incidences based with permission on Needham's plate 19 (Bull. 68, N. Y. State Mus. 1903), those of Nehalennia and of Ischnura kellicotti on Williamson (Ent. News 24: 313. 1913, and 9: Pl. 9. 1898).

Genus: Enallagma Charpentier.

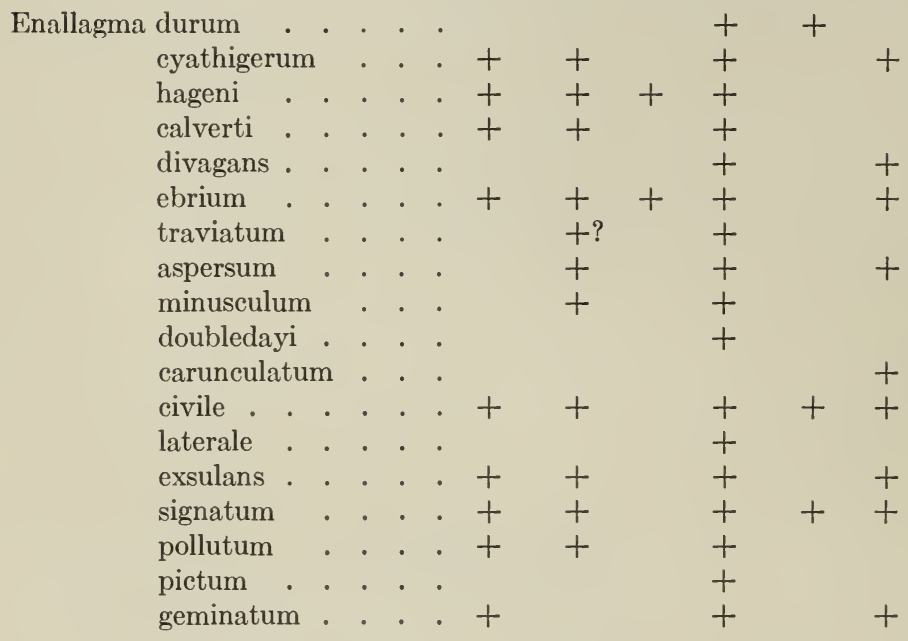

A. 4 or 5 antenodal cells.

\section{Enallagma durum (Hagen).}

Syn. Neur. N. Amer. 87. 1861. Type locality: "Maryland; Louisiana; Florida."

Rare,- - rivers and brooks.

July to August 25.

Massachusetts: Woods Hole, Cuttyhunk Island (Calvert, Ufford).

Rhode Island: Watch Hill (Calvert).

B. 3 antenodal cells.

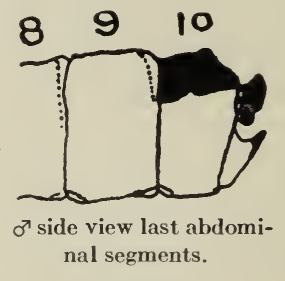

a. $\sigma^{7}$ with abdominal segments 8 and 9 unmarked with black.

\% with black marking on dorsal surface of last abdominal segment.

\section{Enallagma cyathigerum (Charp.).}

Lib. Eur. 163. 1840. Type locality: "Silesia." 
Uncommon,- ponds and lakes.

June to July. 13.

Maine: Manchester (Wadsworth).

New Hampshire: Hermit Lake (Selys);

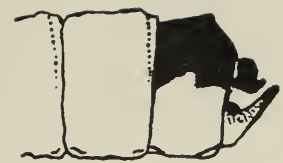

Franconia (Slosson).

Massachusetts: (Selys); Nantucket (Howe).

19. Enallagma hageni (Walsh).

Proc. ent. soc. Phila. 2: 234. 1863. Type locality: [Rock Island, Illinois.]

Common,- - lakes and ponds.

June to August.

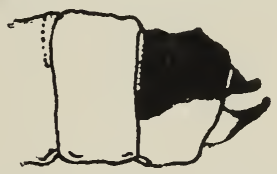

Maine: Foxcroft, Rangeley, Orono, Fryeburg (Harvey); Mt. Desert (Bullock); Manchester (Wadsworth); York Harbor (Moore).

New Hampshire: Meredith, Moultonboro (Howe).

Vermont: Brandon (Dutton).

Massachusetts: Worcester (Hitchings); Dedham (Selys).

20. Enallagma calverti Morse.

Psyche 7: 208. 1895. Type locality: "Franktown, Nev."

Rare,- ponds and marshes.

June 15 to August 12.

Maine: Orono (Harvey).

New Hampshire: Profile Lake (Howe).

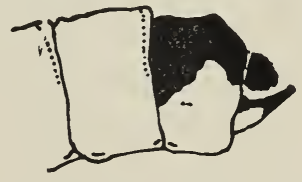

Massachusetts: Wellesley (Morse); Sherborn (Babcock); Concord (Howe).

\section{Enallagma ebrium Hagen.}

Syn. Neur. N. Amer. 89. 1861. Type locality: "Chicago, North America; New Orleans."

Common, - ponds and brooks.

June 21 to August 30.

Maine: Manchester (Wadworth).

New Hampshire: Fabyan's (Calvert); Mere-

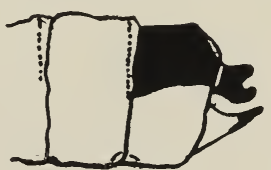
dith Neck, Moultonboro (Howe).

Vermont: Brattleboro (Morse); Brandon (Dutton).

Massachusetts: West Bridgewater (Tower); Concord (Howe); Winchendon, Mt. Everett (B. S. N. H.).

Connecticut: Litchfield (Woodruff). 


\section{Enallagma traviatum Selys.}

Bull. acad. Belg. 41: 517. 1876. Type locality: "Dedham, (Massachusetts)."

Rare,- ponds and lakes.

July 18 to August 19.

New Hampshire: ? ㅇ Moultonboro (Howe).

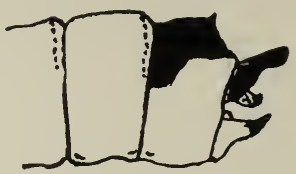

Massachusetts: Dedham (Selys); Sherborn (Morse); Concord (Howe); Hingham (Barnes).

\section{Enallagma aspersum (Hagen).}

Syn. Neur. N. Amer. 97. 1861. Type locality: "New York; Bergen Hill, New Jersey; Chicago."

Uncommon,- ponds.

July to September 5.

Maine: Norway (M. C. Z.).

New Hampshire: North Conway (Calvert).

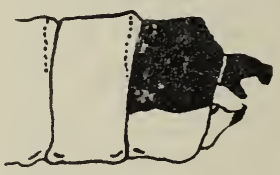

Massachusetts: Nonamesset Island, Woods Hole (Calvert); Concord, Nantucket (Howe).

Connecticut: Fairfield Co. (U. S. N. M.).

\section{Enallagma minusculum Morse.}

Psyche 7: 207. 1895. Type locality: "Sherborn, Mass."

Rare,- lakes and ponds.

July to August 23.

New Hampshire: Meredith Neck (Howe).

Massachusetts: Sherborn (Morse).

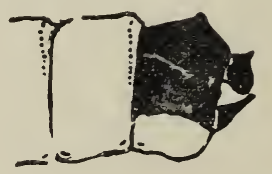

25. Enallagma doubledayi Sely's.

Rev. odon. Eur. 209. 1850. Type locality: "St. John Bluff, en Floride."

Rare, - ponds.

August.

Massachusetts: Provincetown (Williamson); Nonamesset lsland (Calvert).

26. Enallagma carunculatum Morse.

Psyche 7: 208. 1895. Type locality: "Franktown, Nev."

Connecticut: Litchfield (IToodruff).
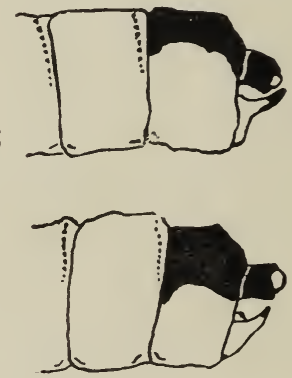


\section{Enallagma civile (Hagen).}

Syn. Neur. N. Amer. 88. 1861. Type locality: "New York," etc.

Abundant,- - lakes, ponds.

May to September 5.

Maine: Orono (Harvey); Manchester (Wadworth); York Harbor (Moore).

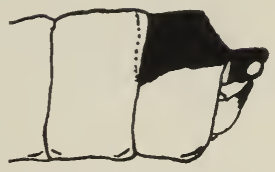

New Hampshire: Lake Asquam, Meredith Neck, Moultonboro (Howe).

Massachusetts: Sherborn (Babcock); Worcester (Hitchings); Woods Hole, Cuttyhunk Island (Calvert); Martha's Vineyard (Moore); Concord, Nantucket, Wareham, Monument Beach (Howe).

Rhode Island: Providence (Davis) Watch Hill, Block Island (Calvert); Bristol, Middletown (Howe).

Connecticut: Litchfield (Woodruff).

aa. $\quad$ with last abdominal segment unmarked with black.

28. Enallagma divagans Selys.

Bull. acad. Belg. 41: 521. 1876. Type locality:- "Derdam, [= Dedham] Massachusetts."

Rare, - ponds.

Massachusetts: Dedham.

Connecticut: Litchfield (Woodruff).

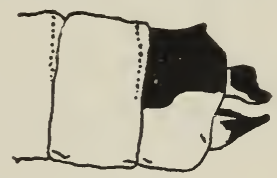

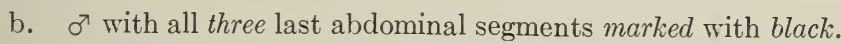
o with last abdominal segment unmarked with black.

29. Enallagma pictum Morse.

Psyche 7: 274. 1895. Type locality: "Sherborn, Mass."

Very rare,- ponds and ditches.

Massachusetts: Sherborn (Babcock).

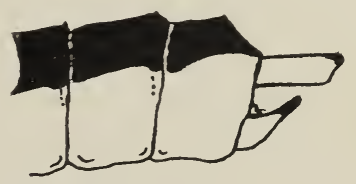

bb. $\quad$ o with ninth abdominal segment entirely black.

30. Enallagma geminatum Kellicott.

Ent. news 6: 239. 1895. Type locality: "Licking Reservoir, Ohio [etc.]...Corunna, Mich."

Uncommon,- - brooks, ponds and lakes. June to September 10.

Maine: Bradley (Harvey).

Massachusetts: Concord (Howe).

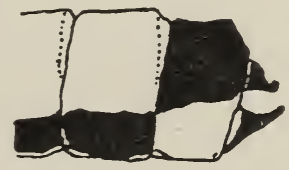


ruff).

Connecticut: Mt. Carmel, New Haven (Moore); Litchfield (Wood-

c. $\sigma^{7}$ with abdominal segment 9 unmarked with black. o with last abdominal segment marked with black.

\section{Enallagma laterale Morse.}

Psyche 7: 274. 1895. Type locality: "Wellesley, Mass."

Rare,- ponds.

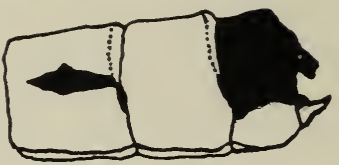

May 25 to July.

Massachusetts: Wellesley (Morse); Concord (Howe).

cc. $\quad$ क with last abdominal segment unmarked with black.

32. Enallagma exsulans (Hagen).

Syn. Neur. N. Amer. 82. 1861. Type locality: "Philadelphia; Berkeley Springs, Virginia; Pecos River, Western Texas."

Common,- brooks, ponds and lakes. June to August 7.

Maine: Orono (Harvey); Manchester (Wadsworth).

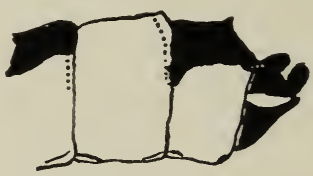

New Hampshire: Meredith Neck (Howe).

Massachusetts: Sherborn, Medfield (Morse).

Connecticut: Litchfield, West Hartford (Woodruff).

33. Enallagma signatum (Hagen).

Syn. Neur. N. Amer. 84. 1861. Type locality: "Georgia; Louisiana."

Common,- - ponds, semicrepuscular. July 2 to September 2.

Maine: Bradley (Harvey); Manchester (Wadsworth).

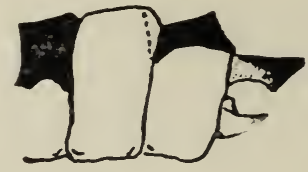

New Hampshire: Meredith Neck, Moultonboro (Howe).

Massachusetts: Worcester (Hitchings); Concord, Woods Hole (Howe).

Rhode Island; Block Island (Woodruff); Middletown (Howe).

Connecticut: West Hartford (Woodruff).

34. Enallagma pollutum (Hagen).

Syn. Neur. N. Amer. 83. 1861. Type locality: "Florida."

Uncommon,- ponds, semi-crepuscular. 
June 23 to August 25.

Maine: Bradley (Harvey); Manchester (Wadsworth).

New Hampshire: Moultonboro (Howe).

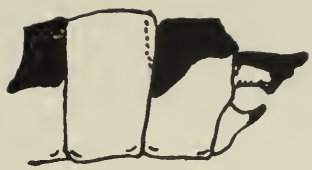

Massachusetts: Concord (Howe).

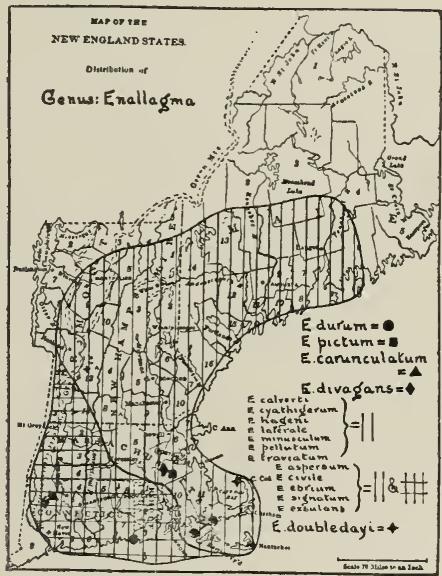

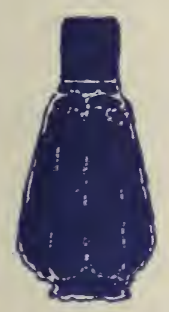

3

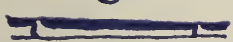

Nehalennia

$\sigma$ and $\$$ bronze green above, abdomen slender.

\section{Group 4}

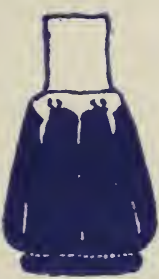

3

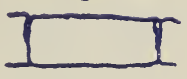

Amphiagrion

$\sigma^{7}$ and $\%$ red and black above, abdomen stout.

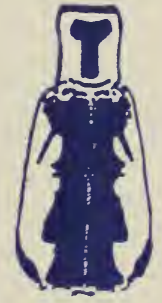

Chromagrion

$\sigma^{7}$ and $\%$ blue and black above.

Color pattern of thorax and base of abdomen of $\sigma^{7}$ 's as seen from above, (also 3rd segment drawn to scale).

Nehalennia irene

Me. N. H. Vt. Mass. R. I. Conn. gracilis

$$
+++
$$$$
+
$$

35. Nehalennia irene (Hagen).

Syn. Neur. N. Amer. 74. 1861. Type locality: "Chicago ... Maine." Uncommon,- grassy ponds and bogs. 
June 24 to August 5 .

Maine: Orono, Fryeburg, Westbrook (Harvey); Manchester (Wadsworth); York Harbor (Moore).

New Hampshire: Meredith, Moultonboro (Howe).

Vermont: Brandon (Dutton).

Massachusetts: Worcester (Hitchings);

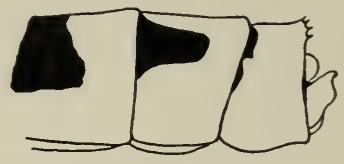
Concord, Nantucket (Howe); Mt. Everett, Chester (B. S. N. H.).

Connecticut: Litchfield (Woodruff).

\section{Nehalennia gracilis Morse.}

Psyche 7:274. 1895. Type locality: "Sherborn [and ] Wellesley, Mass."

Very rare,- - grassy ponds, bogs.

June 19 to July 19.

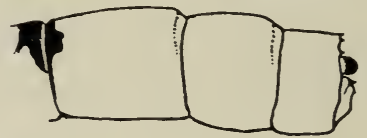

Massachusetts: Wellesley, Sherborn (Morse).

Genus: Amphiagrion Selys

Amphiagrion Me. N. H. Vt. Mass. R. I. Conn.

37. Amphiagrion saucium (Burm.).

Handb. ent. 2:819. 1839. Type locality; "Süd-Karolina."

Rare,- ponds and wet meadows. June to July.

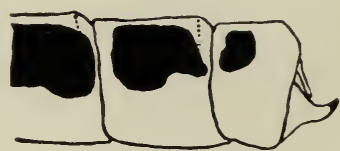

Maine: Orono, Bradley (Harvey); Manchester (Wadsworth).

New Hampshire: Franconia (Slosson).

Vermont: Leicester (Dutton); Montgomery (B. S. N. H.).

Massachusetts: (Hagen).

Genus: Chromagrion Needham.

Chromagrion conditum

Me. N. H. Vt. Mass. R. I. Conn.

38. Chromagrion conditum (Hagen).

Bull. acad. Belg., 41: 1305. 1876. Type locality: "Maryland.... Washington."

Uncommon,- - ponds and brooks.

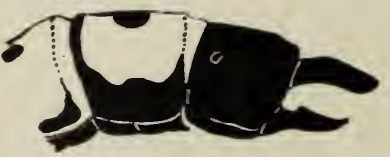
May to July 7 . 
Maine: Orono, Bradley (Harvey); Manchester (Wadsworth).

New Hampshire: Franconia (Slosson).

Massachusetts: Sherborn (Babcock); Concord (Howe).

Connecticut: Litchfield (Woodruff).

\section{Group 5}

Ischnura

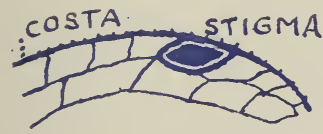

Pterostigma of male touching the costa on fore wings.

of's with more than 7 postcubitals.
Anomalagrion

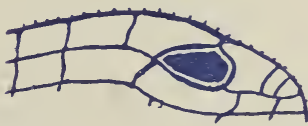

Pterostigma of male not touching the costa of fore wings. ᄋ's with less than 7 postcubitals.

Genus: Ischnura Charp.

\section{Ischnura verticalis kellicotti ramburii posita}

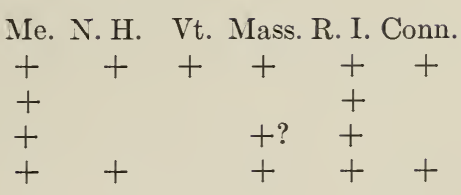

39. Ischnura verticalis (Say).

Journ. acad. nat. sci. Phila. 8: 37. 1839. Type locality: "Indiana."

Abundant,-grassy ponds, brooks and ditches.

May to September 26.

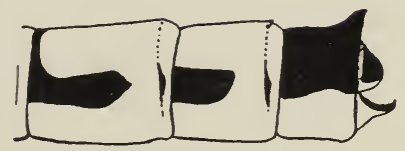

ㅇ dichromatic during growth.

Maine: Orono, Fryeburg (Harvey); Mt. Desert (Bullock); Manchester (Wadswroth); York Harbor (Moore).

New Hampshire: Fabyan's (Calvert); Meredith Neck, Centre Harbor, Moultonboro (Howe).

Vermont: Newport (Slosson); Brandon (Dutton).

Massachusetts: Worcester (Hitchings); Woods Hole, Cuttyhunk Island (Calvert); Martha's Vineyard (Moore); Concord, Nantucket Monument Beach (Howe); Bradley, Provincetown (B. S. N. H.);

Rhode Island: Providence (Davis); Block Island (Calvert); Middletown (Howe).

Connecticut: Poquonock (Britton); South Manchester (Sturgis); Mt. Carmel (Moore); Litchfield (Woodruff); Middletown (B. S. N. H.). 
40. Ischnura kellicotti Williamson.

Ent. News 9: 209. 1898. Type locality: "Round and Shriner Lakes, Whitley County, Indiana.".

Rare,- lakes and ponds.

July to August.

Maine: Manchester (Wadsworth).

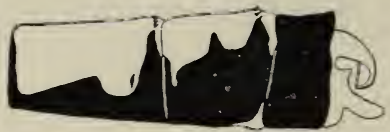

Rhode Island: Block Island (Calvert).

\section{Ischnura ramburii Selys.}

Rev. odon. Eur. 186. 1850. Type locality: "Yucatan."

Rare,- ponds, ditches, and salt marsh borders August to October 2.

?Massachusetts: Canton (M.C.Z.)

Rhode Island: Watch Hill (Calvert); Bristol, Middletown (Howe).

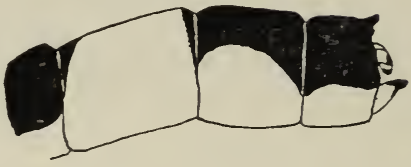

\section{Ischnura posita (Hagen).}

Syn. Neur. N. Amer. 77. 1861. Type locality: "Savannah, Dalton, Georgia; Washington."

Uncommon,- grassy ponds.

June to September 3.

Maine: Orono, Penobscot River (Harvey).

New Hampshire: White Mts. (M. C. Z.).

Massachusetts: Salem (Selys); Nan-

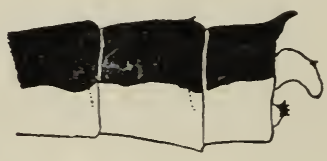
tucket, Concord (Howe).

Rhode Island: Providence (Davis); Block Island (Calvert); Middletown (Howe).

Connecticut: Litchfield (Woodruff).

Genus: Anomalagrion Selys.

Me. N. H. Vt. Mass. R. I. Conn.

Anomalagrion hastatum

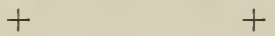

43. Anomalagrion hastatum (Say).

Journ. acad. nat. sci. Phila. 8: 38. 1839. Type locality: "Indiana."

Very rare,-marshes and swamps.

Maine: (Hagen).

Massachusetts: (Hagen).

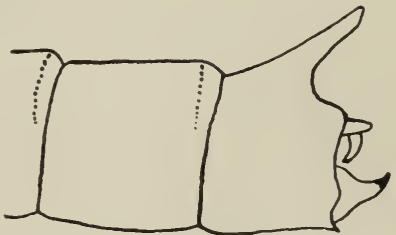


ODONATA

Zygoptera: Damsel Flies

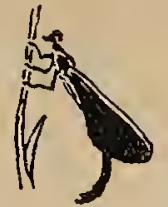

Wings closed when alighted.

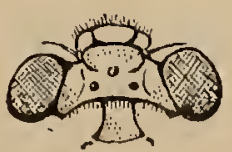

Eyes widely separated, by more than the full width of an eye.

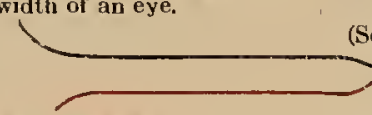

Agrionidae

IANTECUBITALS

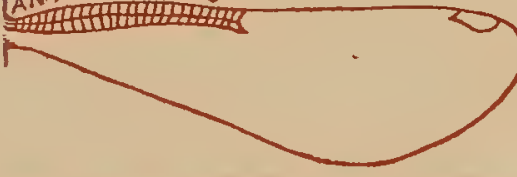

Five or more autecuhitals. Wings nol stalked.

Agrioninae
Agrion

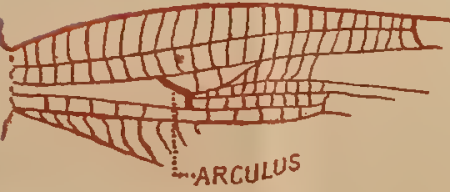

Ar.ARCuLU
Hetaerina

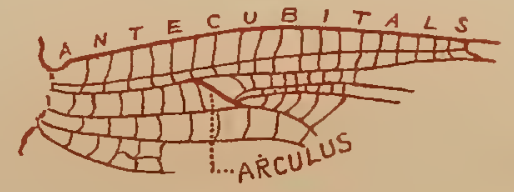

A rculus slmight.

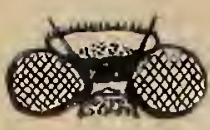

Wiugs open when alighted.

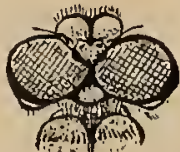

yes approximale, separated by less than half the width of an eye.

(Seer key to Anisoptera: Dragon Flies.)

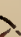

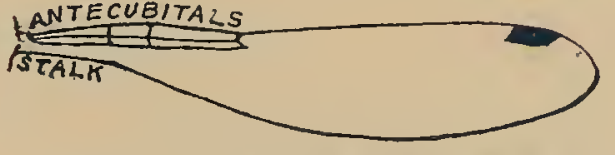

Furn (rarcly three) antecubitals. Wings stolker.
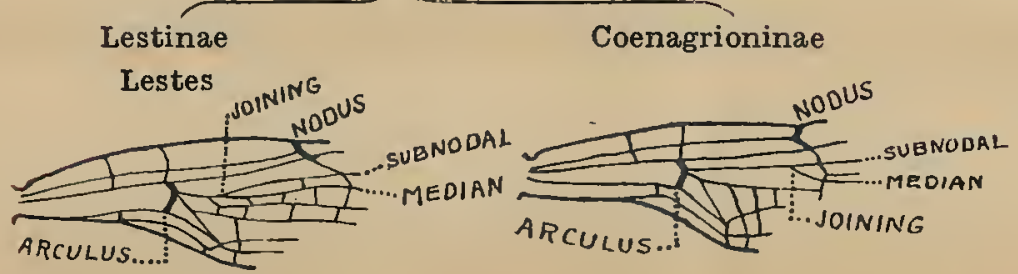

Sulunodal and usctian veins joincin!

Subnotal and uedian veins joinin! neterer the nodus than the arruthe. nemere the arrulus than the jodks.

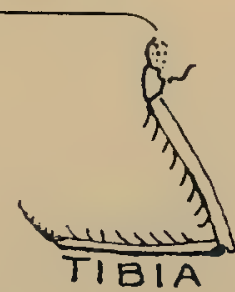

Hairs on tibia twice as long as the spaces hetween the hairs.

llairs on tibia never twice as tong in the spaces between the hairs.

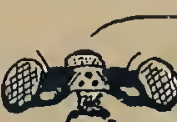

I'ale spots (rarely connected by piste narrow line) on top of head.

\section{Enallagma}

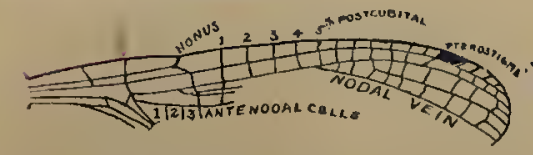

Nodal vein of fore-wiug arising near of leyond 5th postrwhitol.
Nodhal vein of fore-wing arising not licyond 4 th postcubital.

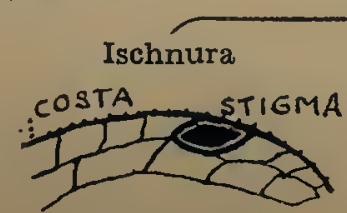

I'lerostigma of mule touchint] the costa on fore wings. Q's with more than 7 mestentitnle.

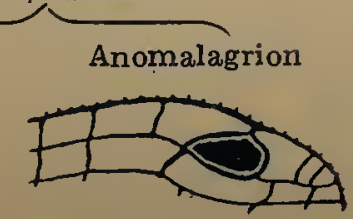

l'terostigma of male not touching the costa of fore wings. Q's wilh lesw then 7 mostcubitats.

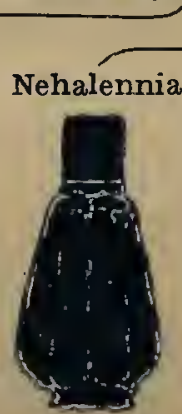

3

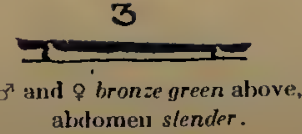

alutomen stender.

Color pattern of thorax and base of abdemen of $\sigma^{x} \mathrm{~s}$ as seen from ahowe ietso 3rd segment of $Q$ 's shdomen showing relation dismerter drawn to seale. 


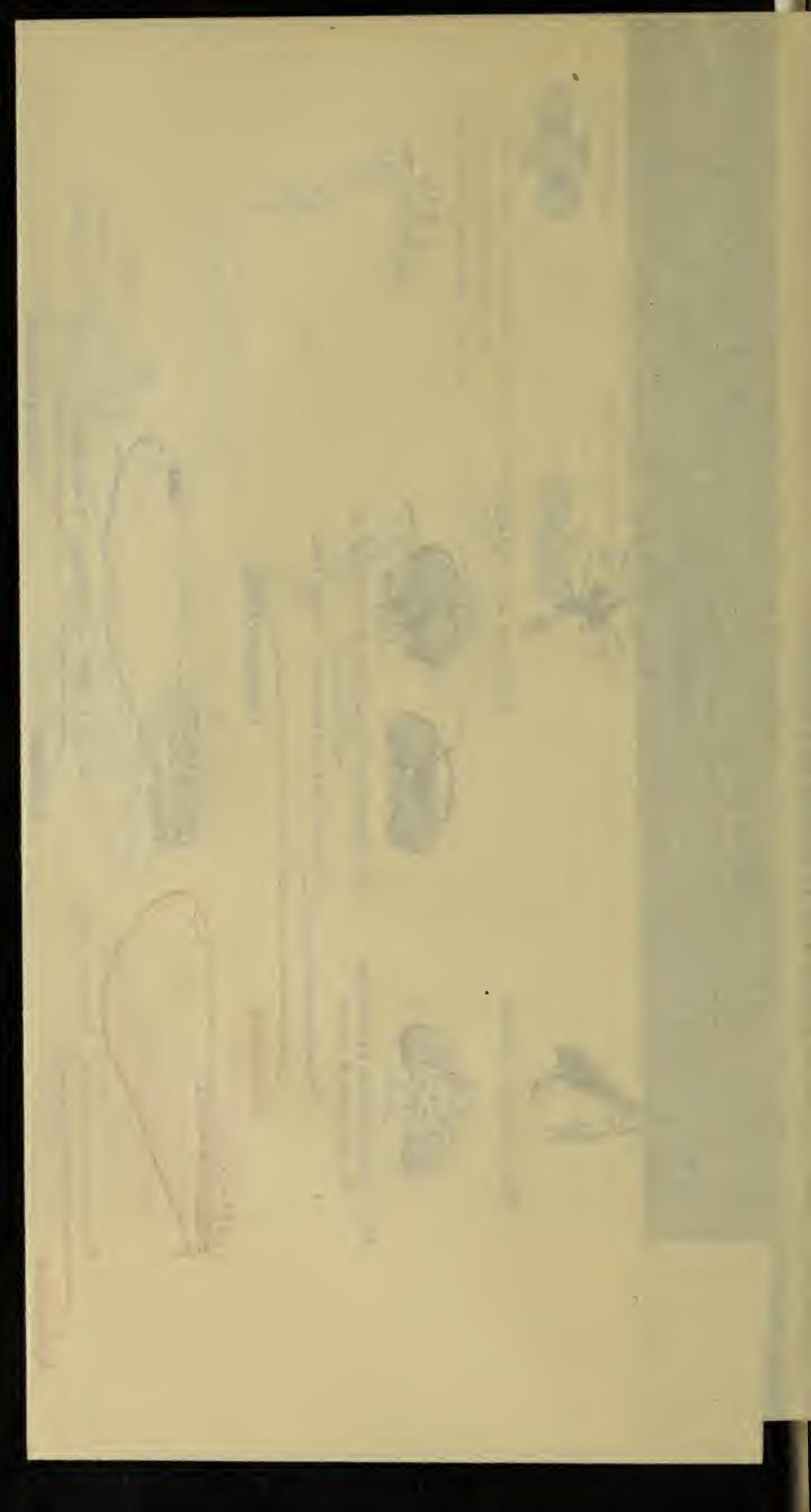



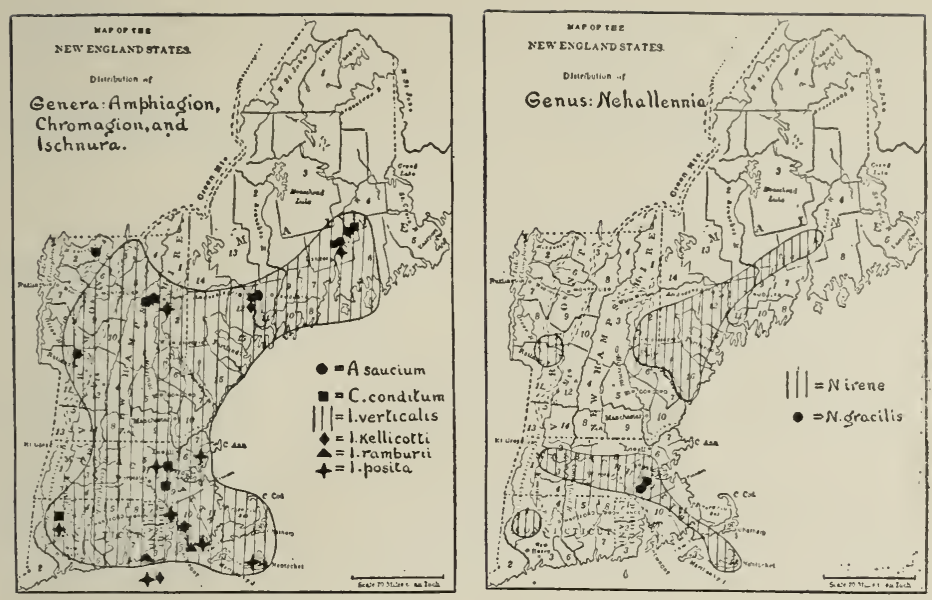

\section{Recapitulation of New England Zygoptera-Damsel-flies}

States

$$
\text { ২ }
$$

Maine

New Hampshire

Vermont

Massachusetts

Rhode Island

Connecticut

$$
\begin{array}{rrrrrrrrrr}
2 & 1 & 8 & 2 & 9 & 1 & 1 & 1 & 4 & 1=30 \\
3 & 0 & 7 & 2 & 11 & 1 & 1 & 1 & 2 & 0=28 \\
2 & 0 & 2 & 1 & 2 & 1 & 1 & 0 & 1 & 0=10 \\
4 & 1 & 9 & 3 & 17 & 2 & 1 & 1 & 3 & 1=42 \\
1 & 0 & 3 & 1 & 3 & 0 & 0 & 0 & 4 & 0=12 \\
3 & 0 & 7 & 2 & 9 & 1 & 0 & 1 & 2 & 0=25
\end{array}
$$

Anisoptera: Dragon Flies.

\section{Aeshnidae}

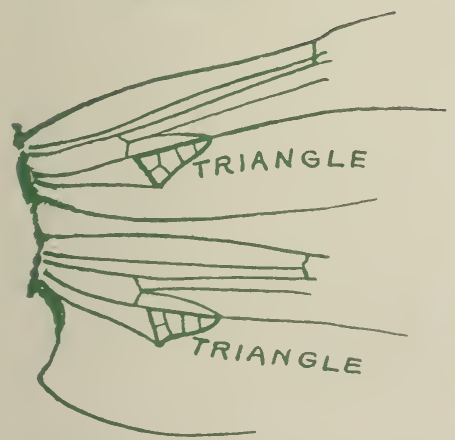

Triangles of fore and hind wings in similar position and of similar shape.

(See Group 1.)
Libellulidae

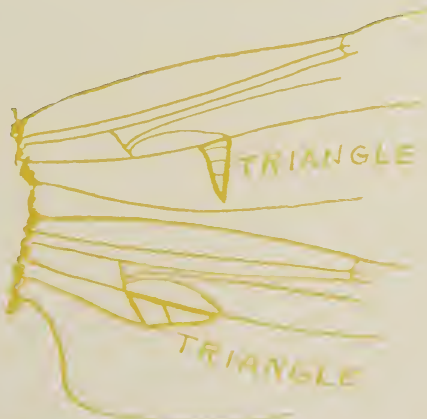


Group 1

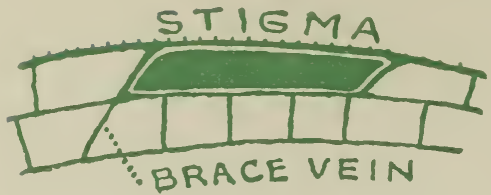

Pterostigma with a brace vein. (See Petalurinae, Gomphinae, and Aeshinae.)
Cordulegasterinae

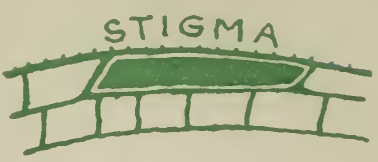

Pterostigma without a brace vein. (See Cordulegasterinaө.)

Petalurinae

Tachopteryx

Group 2

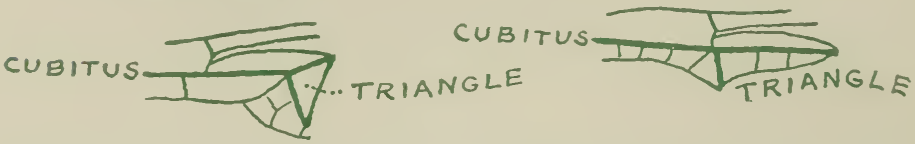

Cubitus appearing symmetrically forked to

form triangle. Subtriangle of three cells.

Cubitus appearing unsymmetrically forked to form triangle. Subtriangle of one cell. (See Gomphinae and Aeshinae.)

Suborder: Anisoptera.

Family: Aeshnidae.

Subfamily: Petalurinae Needham.

Genus: Tachopteryx Selys.

Tachyopteryx thoreyi

Me. N. H. Vt. Mass. R. I. Conn. $\sigma^{7}+$ pterostigma very long $(8-9.5 \mathrm{~mm}$.) and narrow $(.8 \mathrm{~mm}$.).

\section{Tachyopteryx thoreyi (Hagen).}

Monog. Gomp. 373. 18.58. Type locality: "l'Amérique septentrionale?"

Very rare.

June ham).

New Hampshire: Manchester (Burn-

Massachusetts: (Hagen).

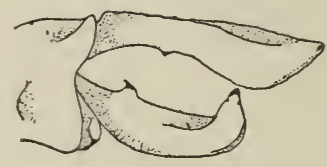

$\sigma^{7}$ side view abdominal appendages.

(To be continued.) 


\section{Memoir of the Thoreau Museum of Natural History: II}

\section{Manual of the Odonata of New England}

By R. HEBER HOWE, Jr.

\section{PART III.}

\section{August 30, 1918.}

Note: Because the distribution of the Gomphines has been so little worked out for the New England States, as evidenced by the few records for each species, the distributional maps have been omitted in this part. The habitats, and limital dates are also very insufficient, but are included wherever possible. No drawings of the male appendages of $G$. adelphus have ever been published, nor am I able to secure a specimen from which to draw them.

\section{Subfamily: Cordulegasterinae Calvert}

Genus: Cordulegaster Leach

$$
\text { Me. N. H. Vt. Mass. R. I. Conn. }
$$

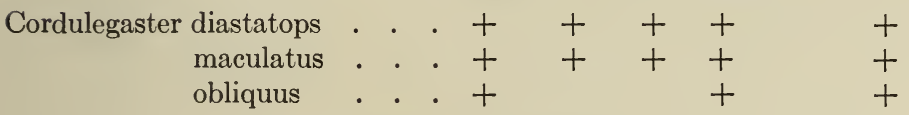

A. Eyes not touching.

45. Cordulegaster diastatops Selys.

Bull. acad. Belg. 21: 101. 1854. Type locality: "Columbie." (Probably error for District of Columbia, U. S.)

Uncommon, - open woods, brooks, and rapid woodland streams.

May 28 to August.

Maine: Greenfield, Bradley, Orono (Harvey); Manchester (Wadsworth); Portland (M. C. Z.).

New Hampshire: White Mts. (Selys); Franconia (Slosson); Waterville (M. C. Z.); Peterboro (Cabot); Jackson (Morse); In- Side view male appendages. tervale (Allen).

Vermont: Jay, Troy (Morse).

Massachusetts: Sherborn (Babcock); West Bridgewater (Tower); Concord (Howe); Framingham (Johnson); Newtonville (Thaxter); Waltham (Bullard); Southbridge (Bromley); Winchendon (Morse).

Connecticut: Litchfield (Woodruff).

B. Eyes touching.

\section{Cordulegaster maculatus Selys.} caine."

Bull. acad. Belg. 21: 105. 1854. Type locality: "Georgie Ameri- 
Uncommon,- open woods, brooks. June 1 to August 7.

Maine: Greenfield, Orono (Harvey); Manchester (Wadsworth).

New Hampshire: Manchester (Burnham); North Conway, Intervale (Allen); Pemigewasset pond; Meredith (Calvert); Franconia (Howe).

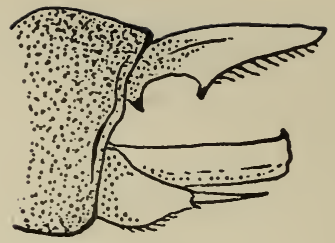

Vermont: Bennington (Johnson).

Massachusetts: Woburn (Hagen); Worcester (Hitchings); West Bridgewater: (Tower); Concord (Howe); Chester (Johnson); Brookline (Shurtleff); Southbridge (Bromley).

Connecticut: (Hagen); Litchfield (Woodruff).

\section{Cordulegaster obliquus (Say).}

Journ. acad. nat. sci. Phila., 8: 15. 1839. Type locality: "Indiana."

Uncommon,- open woods.

May 28 to July 7 .

Maine: Bradley, Orono (Harvey); Manchester (Wadsworth).

Massachusetts : Andover (Selys); Brookline (Hagen); Framingham (Johnson); Southbridge (Bromley).

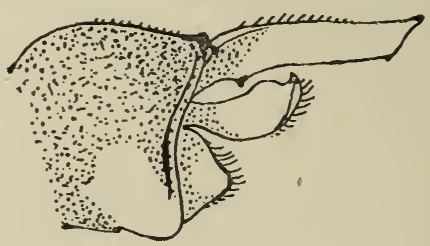

Connecticut: (Hagen).

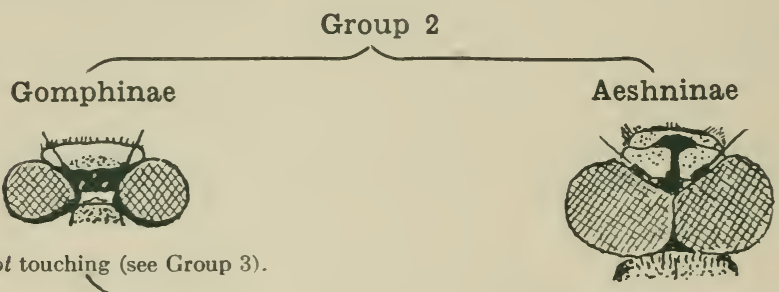

Eyes not touching (see Group 3).

Eyes touching (sce Aeshninae).

Group 3

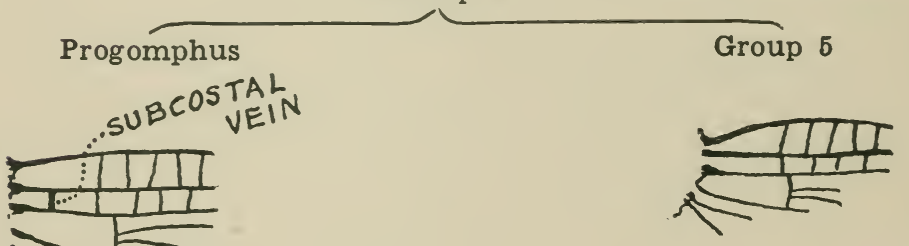

Subcostal vein not present (see Group 5).

Subcostal vein present. 
Subfamily: Gomphinae Rambur

Genus: Progomphus Selys

Progomphus obscurus

Me. N. H. Vt. Mass. R. I. Conn.

48. Progomphus obscurus * Rambur.

Ins. Nevr. 170. 1842. Type locality: "l'Amerique septentrionale." June 5 to August 9.

Very rare,- scrub oak uplands, rivers and lakes.

Massachusetts: Boston, (Hagen); Wareham (Bangs).

$*=$ Gomphoides.

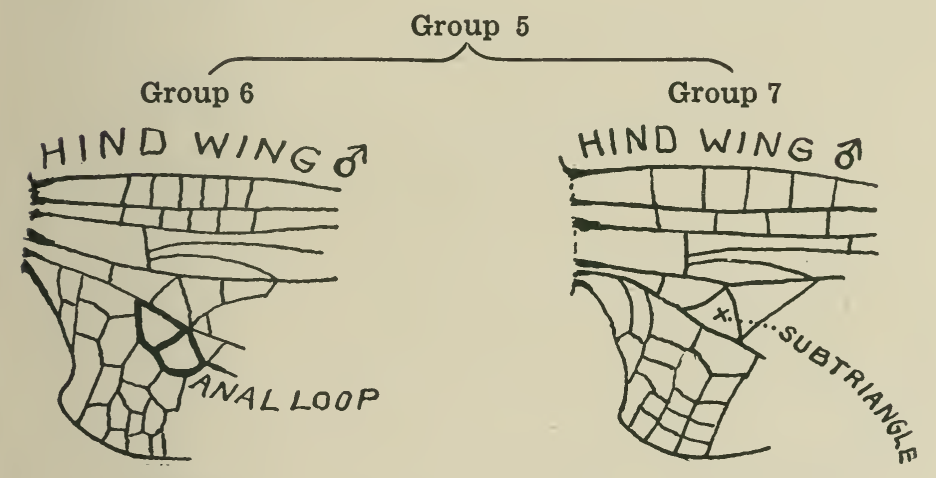

Anal loop present.

\section{Hagenius}

TRIANGLES
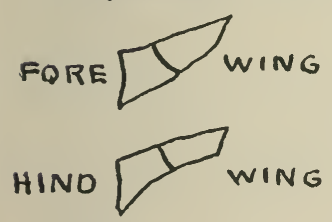

Triangles with cross veins.
Anal loop not present (see Group 7). 


\section{Hagenius brevistylus Selys.}

Bull. acad. Belg. 21: 82. 1854. Type locality: "Columbie" (probably error for District Columbia, U. S.).

Common,-swift mountain brooks, and rivers, ponds and lakes.

June to September 3.

Maine: North East Carry, Greenfield, Orono, Bradley (Harvey); Manchester (Wadsworth).

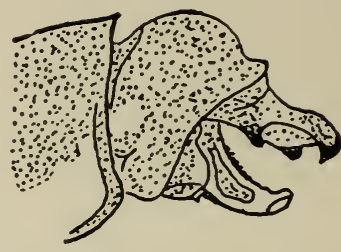

New Hampshire: Manchester (Burnham); Peterboro (Cabot); Meredith Neck, Moultonboro (Howe).

Massachusetts: Sherborn (Babcock); Sutton (Hagen); Concord (Howe); Winchendon (Morse).

Connecticut: Litchfield (Woodruff); Mashapaug (Bromley).

Genus: Ophiogomphus Selys

Me. N. H. Vt. Mass. R. I. Conn.

Ophiogomphus anomalus . . . +

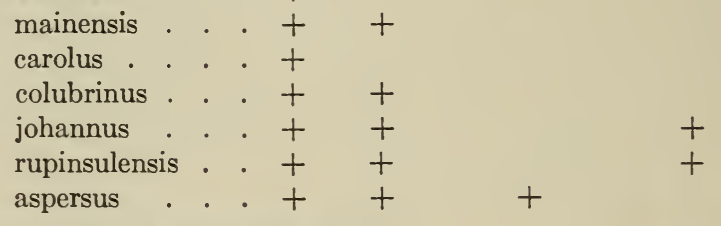

50. Ophiogomphus anomalus Harvey

Ent. News 9: 60. 1898. Type locality: "Orono."

Very rare.

June 6-15.

Maine: Orono (Harvey).

\section{Ophiogomphus mainensis Packard.}

Proc. ent. soc. Phila. 2: 255. 1863.

Type locality: "northern part of the State of Maine."

Very rare,-1400 ft.

Maine: (Packard).

New Hampshire: White Mts. (Selys); Franconia (Slosson).

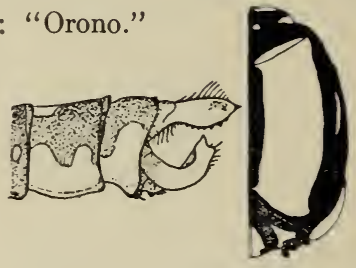

Side view of male abdominal appendages, and thorax as seen from above (both sexes).

Note: The drawing of the male appendages is made from the Hagen type in the Museum of Comparative Zoology - they resemble very closely those of $O$. carolus. 
52. Ophiogomphus carolus Needh.

Can. ent. 29: 183. 1897. Type locality:

"Ithaca, N. Y."

Very rare,-

May to June.

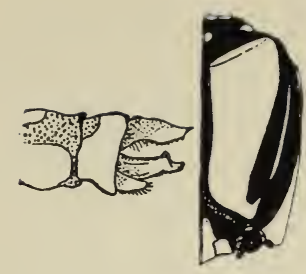

Maine: Greenfield, Bradley, Orono (Harvey).

53. Ophiogomphus colubrinus Selys.

Bull. acad. Belg. 21: 40. 1854. Type locality: "Territoire de la baie d'Hudson."

Rare,-

Maine: Orono (Hagen).

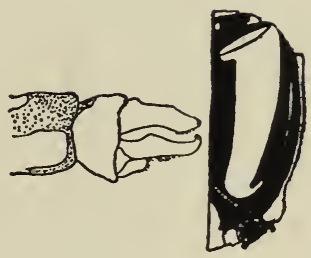

New Hampshire: White Mts. (Banks).

54. Ophiogomphus johannus Needh.

Can. ent. 29: 182. 1897. Type locality: "Wilmurt, N. Y."

Rare,- mountain brooks, and small rivers.

May 27 to July 11.

Maine: Bradley (Harvey).

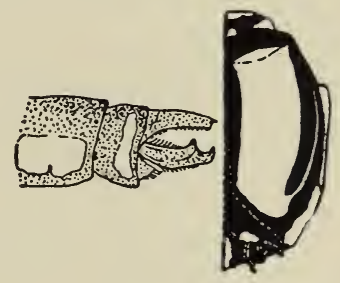

New Hampshire: Merrimac valley (Howe).

Connecticut: Litchfield (Woodruff).

55. Ophiogomphus rupinsulensis (Walsh).

Proc. acad. nat. sci. Phila.; 388. 1862. Type locality: Rock Island, Illinois.

Rare,- small streams, rapid rivers, and windward shores of lakes.

August.

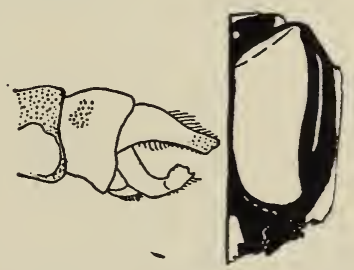

Maine: N. E. Carry, Russell Stream (Harvey); Eagle Lake (M. C. Z.); (Uhler).

New Hampshire: Manchester (Burnham).

Connecticut: New London (Osburn). 


\section{Ophiogomphus aspersus Morse.}

Psyche 7: 209. 1895. Type locality: "northeastern U. S."

Rare,-ledges, woodlands, brooks, 200-2200 ft. alt.

May 26 to August 29.

Maine: N. E. Carry, Russell Stream

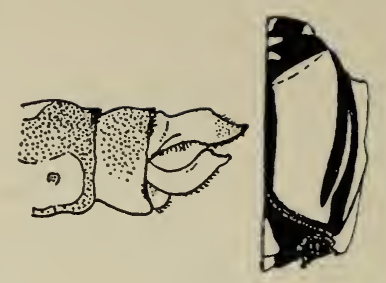
(Harvey).

New Hampshire: Intervale, Mt. Bartlett (Allen).

Massachusetts: Great Barrington (Johnson); Concord (Howe); Wellesley (Morse).

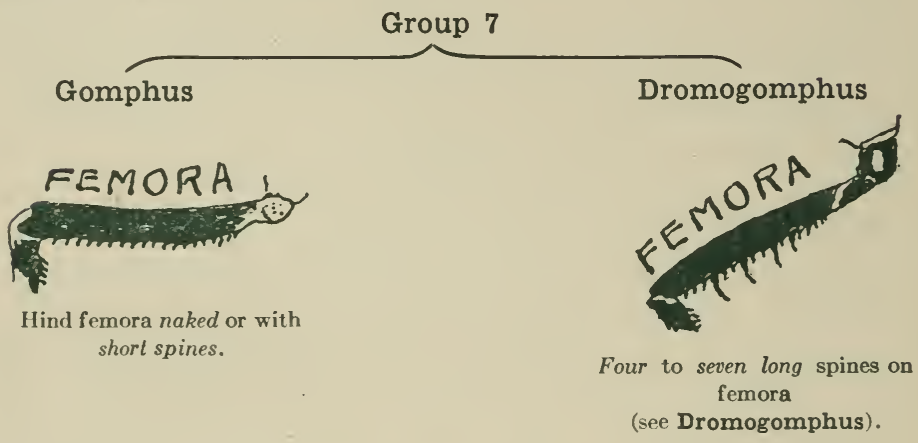

Genus: Gomphus Leach

Me. N. H. Vt. Mass. R. I. Conn.

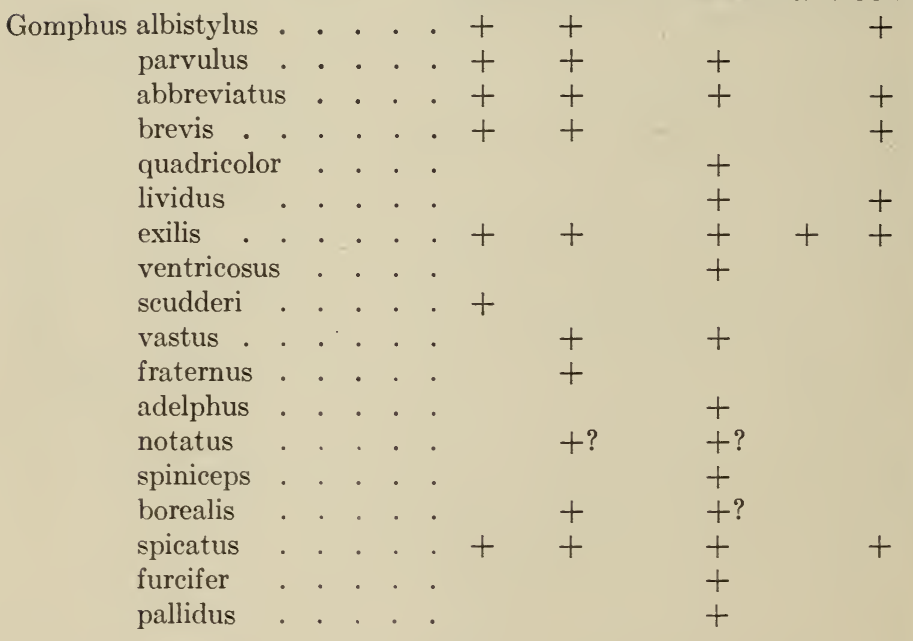


A. Triangle of fore wings distinctly shorter $\left(\frac{1}{3}\right)$ than that of hind wing (=Subgenus: Lanthus Needham. I can see no good reason for recognizing Lanthus as a full genus).

a. Abdomen not over $31 \mathrm{~mm}$. long.

\section{Gomphus albistylus Hagen.}

Bull. acad. Belg. 46: 460. 1878. Type locality: "Le Maine."

Uncommon,- brooks, and small rivers. June 24 to August 1.

Maine: Bradley (Harvey).

New Hampshire: Manchester (Burnham); Franconia (Howe).

Connecticut: Litchfield (Woodruff); New Canaan (Atwater).

\section{Gomphus parvulus Selys.}

Bull. acad. Belg. 21: 56. 1854. Type locality: "Nouvelle-Ecosse."

Rare,-rivers.

May 26 to July 1.

Maine: Manchester (Wadsworth).

New Hampshire: White Mts. (Hagen); Franconia (Slosson, Howe); Peterboro (Cabot).

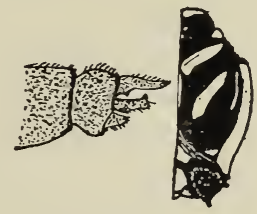

Massachusetts: North Reading, East Walpole (Johnson).

B. Triangle of fore wing only slightly shorter ( $\left.\frac{1}{4}\right)$ than that of hind wing (= Genus: Gomphus).

\section{Gomphus abbreviatus Hagen.}

Bull. acad. Belg. 46: 464. 1878. Type locality: "Provincetown (Massachusetts).... Orono (Maine)."

\section{Uncommon,-}

June 7 to July.

Maine: South Lagrange, Orono (Harvey); Manchester (Wadsworth).

New Hampshire: Manchester (Burnham).

Massachusetts: Provincetown (Hagen).

Connecticut: Winnipauk (Johnson).

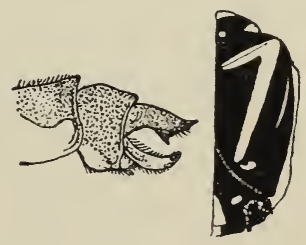


60. Gomphus brevis Hagen.

Bull. acad. Belg. 46: 462. 1878. Type locality: "Schoarie (Etat de New-York)... Port-Neuf (Canada)."

Uncommon,- brooks and rivers.

May 31 to July 8.

Maine: Orono; Bradley, South Lagrange (Harvey).

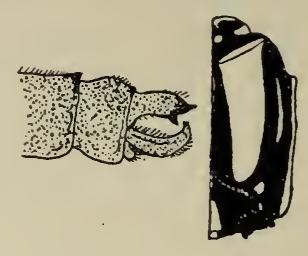

New Hampshire: Intervale (Allen).

Connecticut: Litchfield (Woodruff).

61. Gomphus exilis Selys.

Bull. acad. Belg. 21: 55. 1854. Type locality: "Etat-Unis."

Common,- ponds, slow rivers, woodlands, and roads.

May 19 to August 8.

Maine: South Lagrange, Orono Bradley (Harvey); Mt. Desert (Bullock); Manchester (Wadsworth).

New Hampshire: Manchester (Burnham); Peterboro (Cabot); Meredith Neck, Moultonboro, Franconia, Lisbon (Howe); Inter-

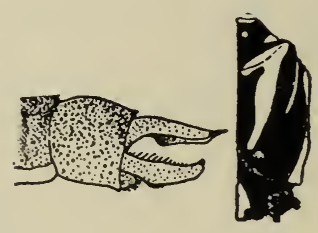
vale, North Conway (Allen); Peterboro (Cabot).

Massachusetts: Salem, Brookline, Natick, Sutton (Hagen); Worcester (Hitchings); Concord (Howe); Newton, Tyngsboro, Framingham, Rutland, North Reading (Johnson); Wareham (Bangs); Medfield, Sherborn (Morse); Provincetown (?).

Rhode Island: Wickford (Johnson).

Connecticut: New Haven (Walden); Litchfield (Woodruff); New London (Osburn).

b. Abdomen between 32 and $38 \mathrm{~mm}$. long.

\section{Gomphus quadricolor Walsh.}

Proc. ent. soc. Phila., 2: 246. 1863. Type locality: Rock Island, Illinois.

Very rare,- gravelly, rocky rivers.

Massachusetts: Mt. Tom (Hagen).

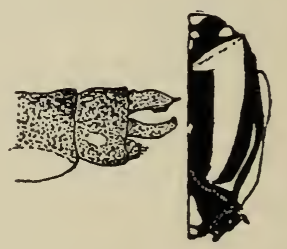

63. Gomphus lividus Selys.*

Bull. acad. Belg., 21: 53. 1854. Type locality: "Caroline du Sud."

Uncommon,- - brooks.

May 28 to July 7

$*=$ sordidus Hagen.

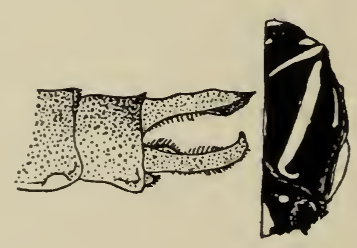


Massachusetts: Natick (Hagen).

Connecticut: Litchfield (Woodruff); New London (Osburn).

64. Gomphus ventricosus Walsh.

Proc. ent. soc. Phila., 2: 249. 1863. Type locality: Rock Island, Illinois.

Very rare,- - rivers.

June 14 to 16 .

Massachusetts: (Selys); Great Barrington (Johnson); Mt. Tom (M. C. Z.).

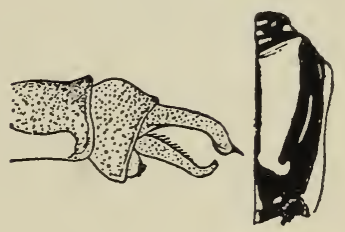

65. Gomphus vastus Walsh.

Proc. acad. nat. sci. Phila, 391. 1862. Type locality: Rock Island, Illinois.

Rare,- rivers and lakes.

July 13 to ham).

New Hampshire: Manchester (Burn-

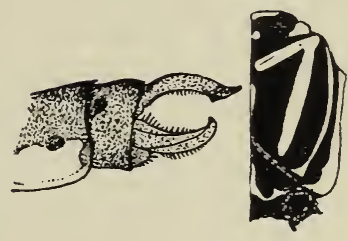

Massachusetts: Tyngsboro (Hagen).

66. Gomphus fraternus (Say).

Journ. acad. nat. sci. Phila., Ser. 1: 16. 1839. Type locality:" U. S."

Very rare,- rivers.

New Hampshire: "Probably" (Hagen).

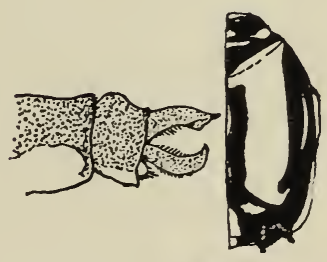

\section{Gomphus adelphus Selys.}

Monog. gomph. 413. 1858. Type locality: "New York."

Very rare,-

Massachusetts: Tyngsboro (Hagen).

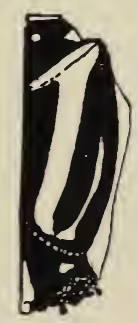

68. Gomphus borealis Needham.

Bull. 47. N. Y. st. mus. 453. 1901. Type locality: "Saranac Inn" N. Y., "Franconia, N. H." 
Rare,-

May to June 21

New Hampshire: Franconia (Slosson); Intervale (Allen).

Massachusetts: ?Concord (Howe).

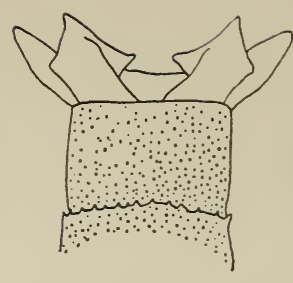

Male appendages from

above

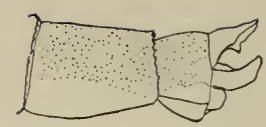

Note: Dorsal thoracic marking same as in following species.

\section{Gomphus spicatus Hagen.}

Bull. acad. Belg. 21: 54. 1854. Type locality: "New York."

Common, about ponds, lakes.

May 15 to July 13

Maine: South Lagrange, Oldtown, Orono (Harvey); Manchester (Wadsworth).

New Hampshire: Franconia (Slosson, Howe); Intervale (Allen); Peterboro (Cabot); Pemigewasset pond
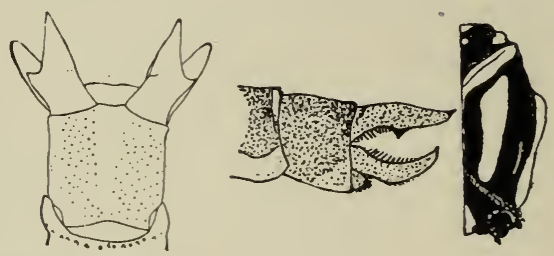
(Calvert).

Massachusetts: Natick (Hagen); Sherborn (Babcock); Concord (Howe); Winchendon (Russell); Boston (Parshley); Bedford (Swett); Southbridge (Bromley).

Connecticut: Litchfield (Woodruff).

Note: Through the kindness of Dr. Calvert, I am able to give figures (side view) of the male appendages of $G$. borealis, and comparative drawings of the appendages of $G$. borealis (from above) and $G$. spicatus (from above). The drawings were made by Dr. Calvert from material in the collection of the Academy of Natural Science, Phila., Pa.

70. Gomphus furcifer Hagen.

Bull. acad. Belg. 46: 458. 1878. Type locality: "Natick (Massachusetts)... . Lansing (Michigan)."

Rare,- open meadows, ponds, and small lakes.

May 29 to July 28.

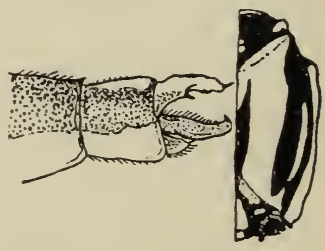

Massachusetts: Natick (Hagen); Southbridge (Bromley); Belmont (Bullard); Tyngsboro (Blanchard); Lexington (Banks); Stockbridge (Calvert); Concord (Howe). 
c. Abdomen between 38 and $44 \mathrm{~mm}$. long.

\section{Gomphus scudderi Selys.}

Bull. acad. Belg. 35: 752. 1873. Type locality: "Etats-Unis."

Rare,- small, rapid rivers.

August 27-29.

Maine: North East Carry (Harvey).

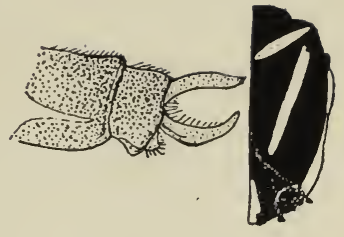

\section{Gomphus notatus Ramb.}

Ins. Nevr. 162. 1842. Type locality: "sans indication de patrie."

Very rare,- large rivers.

Massachusetts: Crampton* (Hagen).

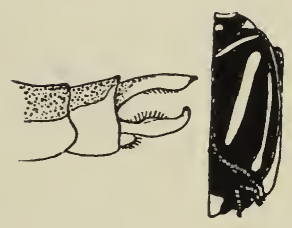

* There is no such town in Massachusetts. The locality intended is probably Campton, N. H.

\section{Gomphus pallidus Ramb.}

Ins. Nevr. 163. 1842. Type locality: "l'Amerique septentrionale."

\section{Rare,-}

Massachusetts: Waltham (Hagen).

d. Abdomen over $46 \mathrm{~mm}$. long.

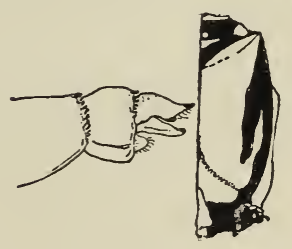

\section{Gomphus spiniceps (Walsh).}

Proc. acad. nat. sci. Phila., 389. 1862. Type locality: Rock Island, Illinois.

Rare,- large rivers. July

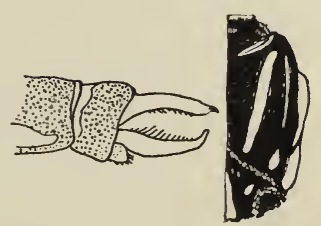

Massachusetts: Lawrence (Hagen); Concord (Howe).

\section{Genus: Dromogomphus Selys}

\section{Dromogomphus spinosus}

Me. N. H. Vt. Mass. R. I. Conn. 


\section{Dromogomphus spinosus Selys.}

Bull. acad. Belg. 21: 59. 1854. Type locality: "Etat-Unis, Georgie."

Common,- - about ponds, lakes, and rivers.

June 28 to September 1.

Maine: Orono, Oldtown (Harvey); Manchester (Wadsworth).

New Hampshire: Manchester, Lake Winnipesaukee (Burnham) Meredith Neck, Newfound Lake, Moultonboro (Howe) Sharon (Cushman); Squam Lake (Allen).

Massachusetts: Worcester (Hitchings); Concord (Howe); Fall River (Easton); Brookline (M. C. Z.); Boston (Allen); Wareham (Bangs); Worcester, Sherborn (Morse).

Connecticut: Litchfield, West Hartford (Woodruff); Mashapaug (Bromley); New London (Osburn).

Subfamily: Aeshninae Rambur

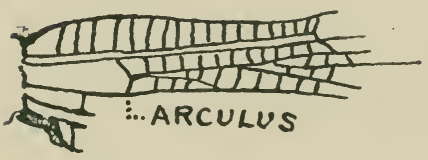

Upper half of arculus not shorter than lower.

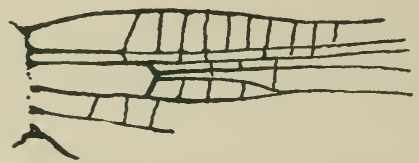

Upper half of arculus shorter than lower. (See Genus: Anax)

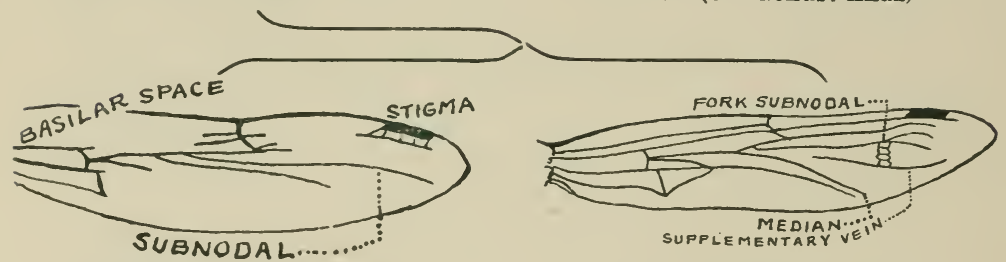

Subnodal (radial) vein unforked.

Subnodal (radial) vein forked. (See Group 1)

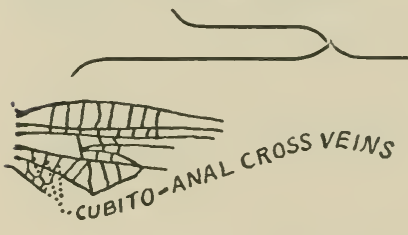

Three or more cubito-anal cross veins.

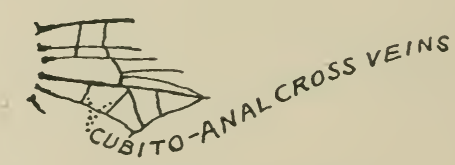

Two cubito-anal cross veins.

(See Genus: Gomphaeschna)

(To be continued) 


\section{Additional Stations and Corrections}

Agrion amatum

Part I

July 29 rivers

Agrion æquabile

May 10 rivers

New Hampshire: Intervale (Allen); Pemigewasset pond (Calvert).

Vermont: Bennington (Johnson); Williamsville (Clench).

Massachusetts: Framingham (Frost); Southbridge(Bromley); Blue Hills (Clench);

Wollaston (M. C. Z.).

Agrion maculatum

New Hampshire: Intervale (Allen); Franconia, Lisbon (Howe); Fitzwilliam (Barnes).

Massachusetts: Southbridge (Bromley); Waltham, Belmont (M. C. Z.); Wellesley (Morse).

Connecticut: New Canaan (Atwater): New Haven (Osburn).

Hetaerina americana

Massachusetts: Southbridge (Bromley); Cambridge (M.C. Z.).

Lestes eurinus

June 5

New Hampshire: North Conway (Allen).

Massachusetts: Southbridge (Bromley); Wayland (Howe).

Lestes congener

New Hampshire: Intervale (Allen).

Massachusetts: East Orleans (Parker); Brookline (Johnson); Woods Hole (Calvert).

Lestes unguiculatus

June 15

Maine: Machias (Johnson).

New Hampshire: Intervale (Allen).

Massachusetts: West Peabody (Little); East Orleans (Parker); Woods Hole, Nonamesset Island (Osburn); Barnstable, Dedham, Eastham, Fall River, Chatham, Great Barrington (Johnson); Horse Neck Beach (Holt).

Rhode Island: Tiverton (Johnson); Bristol (Johnson).

Connecticut: Sachem Head (Calvert); New London (Osburn).

Lestes uncatus

May 30

New Hampshire: Intervale (Allen).

Vermont: St. Albans (Johnson).

Massachusetts: Southbridge (Bromley).

\section{Lestes disjunctus}

Part II

June 21 to September 23

Maine: Machias (Johnson).

New Hampshire: North Conway, Intervale (Allen); Jackson (Skinner); Franconia, Lyman, Lisbon (Howe).

Massachusetts: Provincetown, Martha's Vineyard (Johnson); Southbridge (Bromley).

Lestes forcipatus

Massachusetts: strike out Woods Hole (U. S. N. M.)

Lestes rectangularis

June 20

New Hampshire: Intervale, North Conway (Allen).

Massachusetts: West Peabody (Little); East Orleans (Parker); Eastham, 
Martha's Vineyard, Cheshire (Johnson); Southbridge (Bromley).

Rhode Island: Bristol (Johnson).

Connecticut: Sachem Hcad (Calvert); New London (Osburn).

\section{Lestes vigilax}

May 28

Ncw Hampshire: North Conway, Squam Lake (Allen); Meredith Neck (Howe).

Massachusetts: Framingham (Johnson); Southbridge (Bromley).

Rhode Island: strike out Meredith Neck (Calvert).

Connecticut: Sachem Head (Calvert).

\section{Lestes inequalis}

New Hampshire: Intervale (Allen).

Massachusetts: Southbridge (Bromley).

Rhode Island: Bristol (Johnson).

\section{Argia moesta}

June 16

Maine: Bowlin pond (?); strike out Pudding pond (Allen).

New Hampshire: Squam Lake (Allen); Pudding pond, North Conway (Allen); Fitzwilliam (Barnes).

Massachusetts: Brimfield (Bromley).

\section{Argia violacea}

sandy roads.

New Hampshire: Intervale (Allen); Littleton (Howe); Fitzwilliam (Barnes).

Massachusetts: Plymouth (Johnson); Southbridge (Bromley).

Connecticut: New Canaan (Atwater); New London (Osburn); Sherborn (Morse). Note: fifth line for Williamson read Calvert.

\section{Enallagma durum}

July 23

Massachusetts: Nantucket (Cushman).

\section{Enallagma cyathigerum}

June 25

New Hampshire: Intervale (Allcn); Pemigewasset pond (Calvcrt).

Massachusetts: Provincetown (Johnson); Cotuit (Peirson).

\section{Enallagma hageni}

May 30 to August 6

Maine: Machias, Calais, Princeton (Johnson); Bethel, Norway (M. C. Z.).

New Hampshire: Intervale (Allen); Franconia, Lisbon, Littleton (Howe); No. Conway (M. C. Z.).

Massachusetts: North Reading, Blue Hills (Johnson); Bedford (Swett); Franconia (Howe); Wellesley (Morse); Cambridge, Norfolk Co. (M. C. Z.).

\section{Enallagma calverti}

May 19

Massachusetts: Auburndale (Johnson).

Rhode Island: Matoonoc (Simonds).

\section{Enallagma ebrium}

May 26

Maine: Princeton (Jolınson).

Ncw Hampshire: Franconia, Lyman (Howe).

Vermont: Burlington (Johnson).

Massachusetts: Mt. Greylock (Calvert); Brookline, Washington, Cheshire (Johnson); Southbridge (Bromley); Wayland (Howe); Cambridge, Norfolk Co. (M. C. Z.).

Rhode Island: Bristol (Johnson).

Connecticut: Sachem Head (Calvert); New London (Osburn).

\section{Enallagma traviatum}

July 16

Massachusetts: strike out Hingham (Barnes).

Enallagma aspersum

July 21

New Hampshire: Intervale (Allen). 
Massachusetts: Hingham (Barnes).

Connecticut: Sachem Head (Calvert).

\section{Enallagma doubledayi}

June 29

Enallagma minusculum

June 27

Maine: Echo Lake, Mt. Desert (Johnson).

Massachusetts: Manomet, Eastham (Johnson).

Enallagma carunculatum

July 28

Massachusetts: Stockbridge Bowl (Calvert).

Enallagma civile

September 8

Massachusetts: West Peabody (Little); East Orleans (Parker); Nonamesset Island, No Man's Land (Osburn); Tuckernuck (Allen); Cohasset (Bryant); Fall River (Cushman); Manomet, Eastham, Edgartown (Johnson); Southbridge (Bromley); Natick, Nahant (M. C. Z.); West Chop, Provincetown (Morse).

Rhode Island: Matoonac (Simonds); Bristol (Parker).

Connecticut: Sachem Head (Calvert).

\section{Enallagma divagans}

June 8

New Hampshire: Pemigewasset pond (Calvert).

Massachusetts: North Reading (Johnson); Concord (Howe); Winchendon (Morse).

Connecticut: strike out Litchfield (Woodruff); Middletown (Johnson); New

Canaan (Atwater).

Enallagma geminatum

Massachusetts: Bedford (Swett); Auburndale (Johnson).

\section{Enallagma exsulans}

May 28 to August 27

Massachusetts: Rutland, Framingham (Johnson); Bedford (Swett); Concord

(Howe); Dover (Morse).

Rhode Island: Buttonwoods (Johnson).

Connecticut: Winnipauk (Johnson); New London (Osburn).

\section{Enallagma signatum}

June 17

Maine: Princeton (Johnson).

Vermont: St. Albans (Johnson).

Massachusetts: West Peabody (Little); Brookline (Johnson); Cambridge (M. C.Z.); Cotuit (Peirson); Medfield (Morse).

Connecticut: Winnipauk, Middletown (Johnson).

\section{Enallagma pollutum}

June 8 to September 6

Massachusetts: West Peabody (Little); Brookline (Johnson); Bedford (Swett).

\section{Nehalennia irene}

May 28 to August 23

Maine: Machais, Naples, Calais, Princeton (Johnson); Wales (Frost).

New Hampshire: Intervale, North Conway (Allen); Franconia, Littleton (Howe). Vermont: Burlington (Johnson).

Massachusetts: Framingham, Duxbury, Winchendon, Brookline (Johnson); West Peabody (Little); Mt. Greylock (Calvert); Woods Hole (Osburn); Cambridge, Natick (M. C. Z.); Cotuit (Peirson); Sherborn, Wellesley, Provincetown, Winchendon (Morse).

Rhode Island: Buttonwoods (Johnson).

Connecticut: Sachem Head (Calvert); New London (Osburn).

\section{Amphiagrion saucium}

June 19 to July 16

New Hampshire: Jaffrey (Johnson); Intervale (Allen); Lyman (Howe).

Massachusetts: Wellesley, Sherborn (Morse). 
Rhode Island: Buttonwoods, Bristol (Johnson).

Connecticut: New Canaan (Atwater).

Chromagrion conditum

New Hampshire: Intervale (Allen); Franconia (Howe).

Massachusetts: Duxbury (Johnson); Woods Hole (Osburn); Wellesley (Morse). Ischnura verticalis

May 14

Maine: Ashland Junction, Calais, Princeton, Machias, Bradley (B. S. N. H.); Portland (M. C. Z.); Echo Lake, Mt. Desert, So. West Harbor (Johnson).

New Hamphire: Intervale (Allen); Jackson (Skinner); Franconia, Littleton Lisbon (Howe); No. Conway (M. C. Z.).

Massachusetts: North Reading, Framingham, Blue Hill, Groton, Dedham, Gloucester, Duxbury, Auburndale, Manomet, Washington, Eastham, Edgartown, Horse Neck Beach, Rutland (Johnson); West Peabody (Little); Stockbridge, Mt. Greylock (Calvert); Bedford (Swett); East Orleans (Parker); Tuckernuck (Allen); Southbridge (Bromley); strike out Bradley (B.S. N. H.); Nahant, Cambridge (M. C.Z.); Cotuit (Peirson).

Rhode Island: Tiverton, Washington (Johnson); Matoonoc (Simonds); Bristol (Parker).

Connecticut: New London (Osburn).

Ischnura ramburii

July 20

Massachusetts: Woods Hole (Osborn).

Ischnura posita

May 25 to September 6

New Hampshire: Exeter (Howe).

Massachusetts: Brookline, Dedham, Auburndale, Duxbury (Johnson); Framingham (Frost); Woods Hole (Calvert); Natick (M. C. Z.); Winchendon (Morse).

Rhode Island: Bristol (Parker).

Connecticut: Middletown (Johnson); New Canaan (Atwater).

\section{Anomalagrion hastatum}

June 22 to August 20

Massachusetts: Wellesley (Morse); Woods Hole (Osburn); Dorchest er (M. C. Z.); Concord (Howe).

Rhode Island: Tiverton (Johnson).

Connecticut: Sachem Head (Calvert).

\section{Recapitulation of New England Zygoptera}

Maine

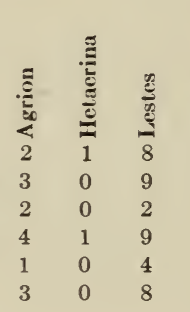

2
2
2
1

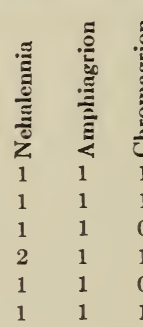

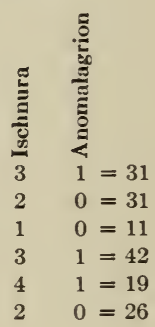




\title{
Memoir of the 'Thoreau Musem of Natural History: II Manual of Odonata of New England
}

By R. HEBER HOWE, Jr.

\author{
March 25, 1919.
}

\section{PART IV}

Note: The drawings are made in many cases from the illustrations in articles hy Drs. E. M. Walker, J. G. Needham, R. A. Muttkowski, C. H. Kennedy, and Mr. E. B. Williamson, and are used here with permission. No distributional maps will be given in the future parts as new stations are so frequently found that maps become obsolete almost as soon as published.

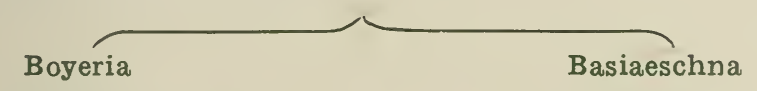

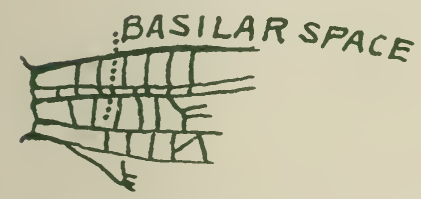

Basilar space with cross veins.

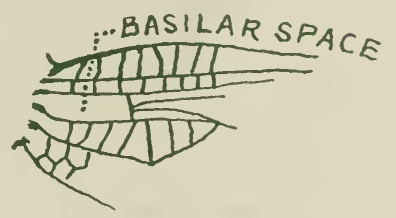

Basilar space wilhoul cross veins. (See Basiaeschna.)

Genus: Boyeria MacLach.

Me. N. H. Vt. Mass. R. I. Conn.

Boyeria grafiana . . . . . + vinosa . . . . . ++++

A. Body markings with yellowish-brown predominating, wings smoky.

\section{Boyeria vinosa (Say).}

Jour. acad. nat. sci. Phila. 8: 13. 1839. Type locality: "Massachusetts."

Uncommon,-—brooks, rivers, lakes,-—preferring shady places or semicrepuscular.

June 19 to September 13.

Maine: No. East Carry, Greenfield, Orono, Wissattiquoik river (Harvey); Mt. Desert (Bullock); Manchester (Wadsworth); 
New Hampshire: Meredith Neck (Howe).

Vermont: Wolcott, Grand Isle (Morse); Brandon (Dutton).

Massachusetts: Concord (Howe); Cambridge (Hagen); Truro, Sherborn (Morse); Chester (Johnson); Fall River (Holt); Wareham (Bangs); Southbridge (Bromley).

Connecticut: Litchfield (Woodruff).

B. Body markings with blue predominating, wings clear.

\section{Boyeria grafiana Williamson.}

Ent. News 18: 1. 1907. Type locality: "Ohio Pyle, Penna." One record,- July 5, 1899,- lakes.

Massachusetts: Cummington (Knab in U. S. N. M.)

\section{Genus: Basiaeschna Sely's.}

Basiaeschna janata

Me. N. H. Vt. Mass. R. I. Conn.

7S. Basiaeschna janata (Say).

Journ. acad. nat. sci. Phila. 8: 13. 1839. Type locality: "Massachusetts."

Common,- brooks, rivers, ponds, and lakes.

May 9 to July 18.

Maine: So. Lagrange, Orono, Bradley, Westbrook, Greenfield (Harvey); Manchester (Wadsworth).

New Hampshire: White Mts. (Hagen); Peterboro (Cabot); Meredith Neck, Echo Lake, Franconia (Howe); Intervale, Squam Lake (Allen).

Vermont: Brandon (Dutton); Wolcott (Morse).

Massachusetts: Roxbury, Milton, Carver Woods (Hagen); Worcester (Hitchings); Fall River (Holt); Concord (Howe); Wareham (Bangs); Newtonville (Morse); Newton (Thaxter); Waltham (Bullard); Southbridge (Bromley).

Connecticut: Litchfield (Woodruff); New London (Osburn).

Genus: Gomphaeschna Selys.

Gomphaeschna furcillata

Me. N. H. Vt. Mass. R. I. Conn. 


\section{Gomphaeschna furcillata (Say)}

Journ. acad. nat. sci. Phila. 8: 13. 1839. Type locality: "Massachusetts."

Rare,- ponds, sphagnum bogs.

May 30 to July.

Maine: Manchester (Wadsworth).

New Hampshire: Jaffrey (Johnson).

Vermont: Newport (Slosson).

Massachusetts: Manchester, Brookline, Milton, Sutton (Hagen); No. Reading (Johnson); Concord (Howe); Sherborn (Babcock); Princeton, Wollaston (Sprague).

Genus: Anax Leach.

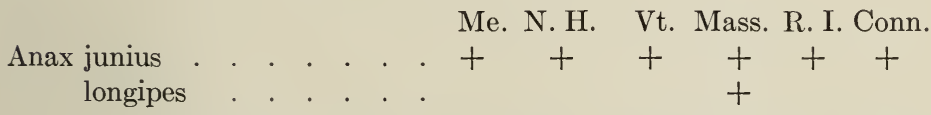

A. Hind wings more than $14 \mathrm{~mm}$. wide. Frons above blue, yellow, and black.

\section{Anax junius (Drury).}

Ill. Nat. Hist. 1: 112. 1770. Type locality: "New York."

$$
\begin{aligned}
& \text { Common,- ponds, migratory } \\
& \text { along coast.* }
\end{aligned}
$$

May 18 to October 13.

Maine: Orono (Harvey); Manchester (Wadsworth).

New Hampshire: Manchester (Burnham); Amherst, Petersboro (Cabot); Meredith, Franconia (Howe).

Vermont: Brandon (Dutton).

Massachusetts: Boston, Cambridge, Springfield (Cabot); Magnolia, Brookline, Milton (Hagen); Woods Hole, Cuttyhunk Island (Calvert); Nantucket, Concord, Scituate (Howe); Fall River (Holt); Blue Hills (Clench); No. Saugus (Webster); Provincetown (Benedict); Wellesley (Morse); Manomet (Cushman); Framingham (Frost); Newtonville (Thaxter); Wareham (Bangs); Minot (M. C. Z.); Southbridge (Bromley). vert).

Rhode Island: Middletown, Bristol (Howe); Block Island (Cal- 
Connecticut: New London (Calvert); Litchfield (Woodruff); Fairfield Co. (Benedict).

B. Hind wings less than $14 \mathrm{~mm}$. wide. Frons above green.

\section{Anax longipes Hagen.}

Syn. Neur. No. Amer. 118. 1861. Type locality: "Georgia."

Rare, - ponds, migratory along coast.

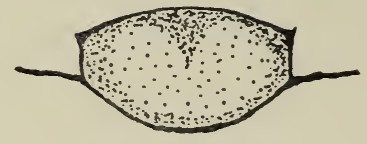

Frons from above.

August 25.

Massachusetts: Woods Hole (Hagen); Wareham (Bangs).

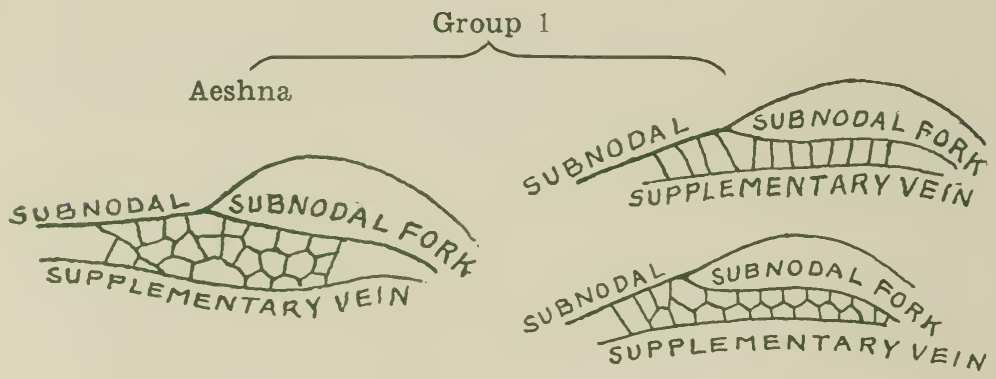

Three to seven rows of cells between subnodal and supplementary veins.

One or luo rows of cells between the subnodal and supplementary veins. (See Group 2.)

Genus: Aeshna Fabricius.

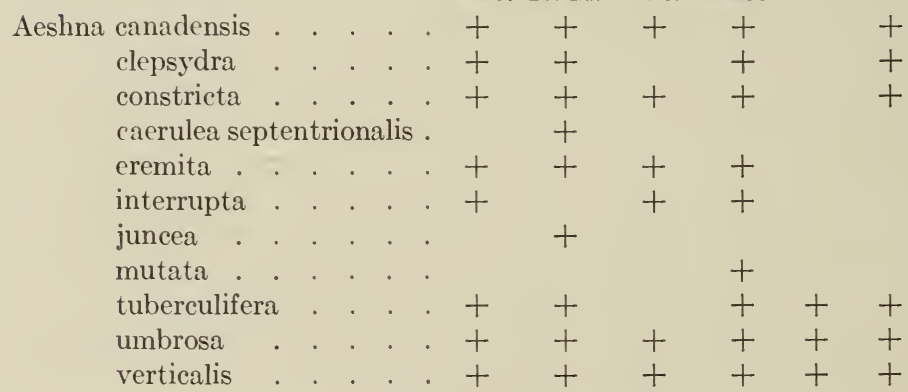

Me. N. H. Vt. Mass. R. I. Conn.

A. Rear of head in part yellowish.

82. Aeshna umbrosa Walk.

Can. Ent. 40: 380, 390. 1908. Type locality: "De Grassi Point, Ont." 
Common, - streams, ponds, lakes, upland meadows.

May 6 to October 31 .

Maine: Manchester, Augusta (Wadsworth); Norway (Smith); Gorham (?): Millinocket, Bradley, Greenfield, Six Ponds (Harvey); Russell Stream (Corry).

New Hampshire: Franconia (Slosson, Howe); White Mts. (Sprague); Hermit lake, Mt. Washington (Scudder); Center Harbor (?); Lake Sun-

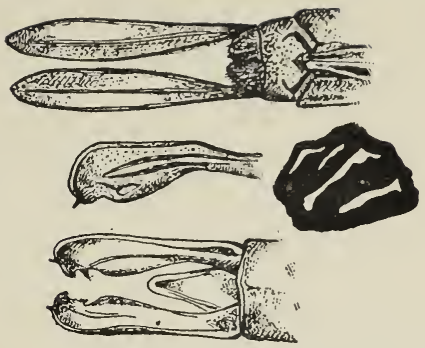

Female abdominal appendages from below. Side, and view from above of male abdominal appendages, and side view of thoracic markings of both sexes. apee, Meredith Neck, Peterboro, Lonesome Lake (Howe).

Vermont: Brandon (Dutton).

Massachusetts: Boston, Cambridge (?); Amherst (Needham); Wilbraham (Martin); Auburndale, Gay Head (?); Mt. Greylock, Stockbridge (Calvert); Sherborn (Frost); Rutland (Johnson); Fall River (Easton); Nonquitt (Baldwin); Belmont, Concord (Howe); Wareham (Bangs); Waltham (Bullard); Southbridge (Bromley).

Rhode Island: Bristol (Parker, Clark).

Connecticut: Litchfield (Woodruff).

B. Rear of head entirely black.

a. Black line across face.

I. Thoracic bands interrupted resulting in spots.

83. Aeshna interrupta Walk.

Can. Ent. 40: 381, 387. 1908. Type locality: "Nipigon, Ont."

Rare,- ponds, lakes.

July 17 to September 15 .

Maine: Portland (Jones).

Vermont (Frost).

Massachusetts: (Uhler); Mt. Greylock (Calvert); Heath (Warden).

II. Thoracic bands not inter-

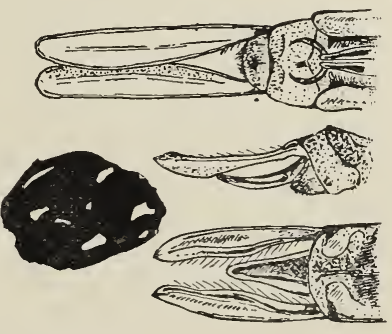
rupted.

1. First thoracic band bent forward at upper end into an anterior spur.

\section{Aeshna clepsydra Say.}

Journ. acad. nat. sci. Phila. 8: 12. 1839. Type locality: "Massachusetts." 
Common, - - ponds, lakes, rivers. July 6 to October 9 .

Maine: Manchester (Wadsworth); Mt. Ktaadin, So. Lagrange, Greenfield, Bradley, Orono (Harvey).

New Hampshire: Moultonboro (Howe); Hermit Lake (Scudder); Fabyan's (Calvert); Manchester (Burnham); No. Conway (Morse).

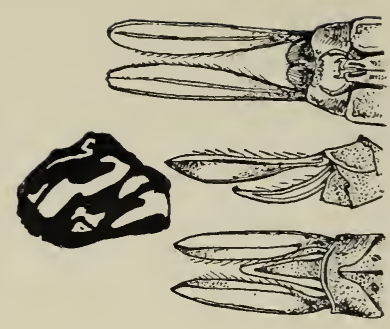

Massachusetts: Sherborn (Babcock); Brookline (Shurtleff); Provincetown (Benedict); Wilbraham (Hagen); Boston (Uhler); Salem (True); Natick (Sanborn); Concord, Nantucket (Howe); Sherborn (Morse); Wareham (Bangs).

Connecticut: Litchfield (Woodruff); New London (Osburn).

2. First thoracic band not bent forward at upper end into an anterior spur.

* First thoracic band straight with borders nearly parallel, without posterior spur at upper end.

85. Aeshna juncea (Linn.).

Sys. Nat. 1: 544. 1758. Type locality: "Europa."

Rare,-

August.

New Hampshire: White Mts. (Hagen, Scudder); Franconia (Slosson).

**First thoracic band straight with borders not parallel, front border excavated, upper end with a detached posterior spur.

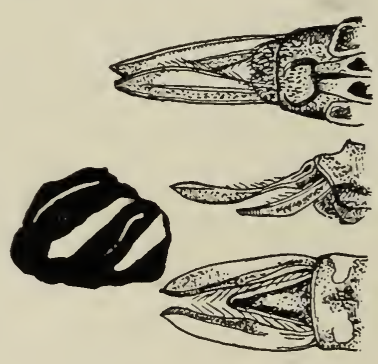

S6. Aeshna eremita Scudder.

Proc. Bost. soc. nat. hist. 10: 215. 1866. Type locality: "White Mts.," Hermit Lake.

Rare,- ponds, lakes.

August 1 to October 1.

Maine: Six Ponds, Piscataquis Co. (Harvey).

New Hampshire: Franconia (Slosson); Hermit Lake, Mt. Washington (Scudder); Profile Lake, Lonesome Lake, Moultonboro (Howe); No. Conway (Holt); Tuckerman's Ravine (Scudder):

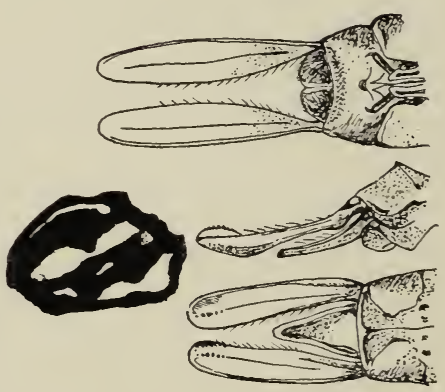


Vermont: Brandon (Dutton).

Massachusetts: Fall River (Holt); Concord (Howe).

*** First thoracic band curved (sigmoid), slender, with detached spots at both ends.

\section{Aeshna caerulea septentrionalis Burm.}

Handb. Ent. 2: 839. 1839. Type locality: "Labrador."

Rare,--

New Hampshire: White Mts. (Scudder, Hagen).

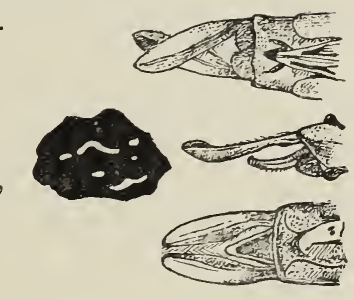

b. No black line across face.

I. First thoracic band without posterior spur at upper end.

88. Aeshna mutata Hagen.

Syn. Neur. No. Amer. 124. 1861.

Type locality: "North America."

\section{Rare,-}

Massachusetts: Wil bra ham (Needham).

II. First thoracic band with posterior spur at upper end.

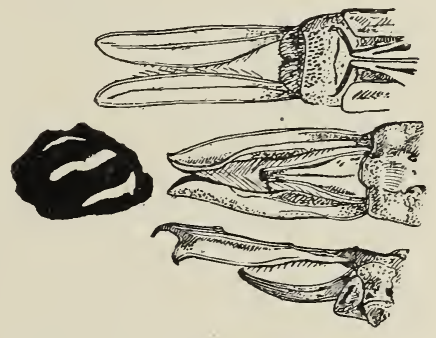

1. First thoracic band with wide, blunt, posterior spur at upper end.

\section{Aeshna verticalis Hagen.}

Syn. Neur. No. Amer. 122. 1861. Type locality: "Washington, New York."

Common,- - ponds, lakes, upland meadows,-migratory along coast.

August 2 to October 2 .

Maine: Manchester (Wadsworth); West Beach (Hagen); Orono, Bradley (Harvey); Manchester (Wadsworth); Islesboro (Dixon); Wells (Deane).

New Hampshire: Meredith Neck (Howe); Manchester (Burnham).

Vermont: Brandon (Dutton).

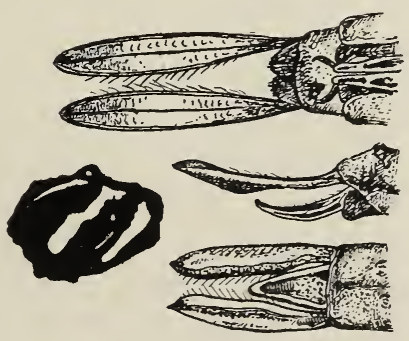


Massachusetts: Beverly, Cambridge, Saugus, Sherborn (?); Nahant (Moring); Salem (Lane); Walpole, Wollaston (Sprague); Westboro (Needham); Kingston, Concord (Howe); Worcester (Hitchings); Provincetown (Morse); Nonquitt (Baldwin); Magnolia (M. C. Z.); Southbridge (Bromley).

Rhode Island: Middletown (Howe).

Connecticut: Litchfield (Woodruff); New London (Osburn)

2. First thoracic band with slender, acute posterior spur at upper end.

* First thoracic band straight (stout), with borders nearly parallel.

90. Aeshna tuberculifera Walk.

Can. Ent. 40: 385, 387. 1908. Type locality: "Isleboro, Me."

Lncommon,- ponds.

August 5 to September 22.

Maine: Islesboro (Dixon); Manchester (Wadsworth).

New Hampshire: North Conway (Holt).

Massachusetts: Walpole (Sprague); Essex Co. (Hagen); Provincetown (Benedict, Zabriskie); Hamp-

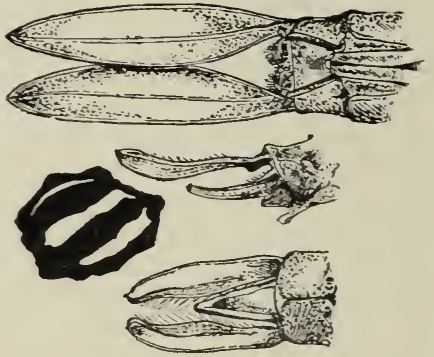
den, Westboro (Needham); Concord (Howe); Dedham, Rutland (Johnson).

Rhode Island: Bristol (Howe).

Connecticut: Litchfield (Woodruff).

** First thoracic band with borders not parallel.

$\dagger$ First thoracic band with front border only excavated.

91. Aeshna canadensis Walk.

Can. Ent. 40: 3S2. 190S. Type locality: "De Grassi Point, Ont."

Common, - rivers, ponds, lakes, and meadowlands.

July 20 to September 26.

Maine: Bradley, Orono, Six Ponds, Piscataqua Co. (Harrey); Manchester (Wadsworth); Norway (Nmith); West Beach (M. C. Z.).

New Hampshire: White Mts. (Shurtleff, Calvert); Franconia (Calvert); Fabyan's (?); Moultonboro, Lyman, Meredith Neck, Jackson (Howe); Fitzwilliam

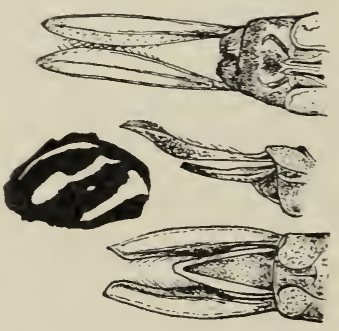


(Barnes); Hermit Lake, Mt. Washington, Carter's Notch, Intervale, No. Conway (Allen).*

* See under A. eremila, Can. Ent. 51: 13. 1919.

Vermont: Newport (Slosson); Brandon (Dutton).

Massachusetts: (Needham); Concord (Howe); Dedham, Boston (Johnson); Mt. Greylock (Calvert); Wareham (Bangs); Beverly, Nahant (M. C. Z.).

Connecticut: Litchfield (Woodruff).

$\dagger \dagger$ First thoracic band with both borders excavated.

\section{Aeshna constricta Say.}

Journ. acad. nat. sci. Phila. 8: 11. 1839. Type locality: "Indiana."

Common,- ponds, rivers.

June 19 to October.

Maine: No. East Carry, Mt. Kineo, Mt. Ktaadin, Orono, Fryeburg (Harvey); Manchester (Wadsworth).

New Hampshire: Hermit Lake, Fabyan's (Calvert); Manchester (Burnham); Intervale, Carter's Notch (Allen).*

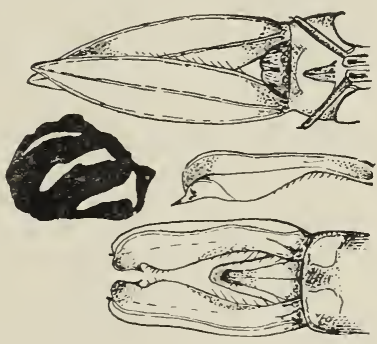

Vermont: Winooski (Davis); Brandon (Dutton).

Massachusetts: Boston (Cabot); Worcester (Hitchings); Auburndale (Johnson); Concord (Howe); Fall River (Holt); Hampden (Needham); Wellesley (Morse); Southbridge (Bromley).

Connecticut: (Hagen); Litchfield (IVoodruff); New London(Osburn).

* The mountain records quite likely refer to $A$. umbrosa, and were made before the species was described.

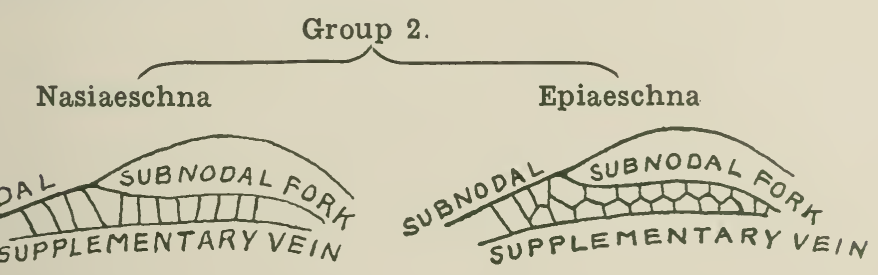

SUPPLEMENTARY VEIN
With one row of cells between subnodal and supplementary veins.
With two rows of cells between subnodal and supplementary veins.

(See Epiaeschna).

Genus: Nasiaeschna Selırs.

Nasiaeschna pentacantha . . Me. N. H 
93. Nasiaeschna pentacantha (Ramb.).

Ins. Neur. 208. 1842. Type locality: "Neuvelle-Orléans."

Rare,- streams and lakes,- one record.

July 9, 1916.

New Hampshire: Moultonboro (Howe).

Genus: Epiaeschna Hagan.

Epiaeschna heros . . . . . +++

94. Epiaeschna heros (Fabr.).

Ent. syst. suppl. 285. 1798. Type locality: "America boreali."

Common,- - ponds, lakes, uplands,- - largely maritime.

May 25 to September.

Maine: Bradley (Harvey); Manchester (Wadsworth); Portland (Cabot); York Beach (Burnham).

New Hampshire: Manchester, Hampton (Burnham).

Massachusetts: Manchester, Nahant, Milton (Hagen); Worcester (Hitchings); Marthas Vineyard (Moore); Fall River (Holt); Brookline, Concord, Hull (Howe); Boston (Johnson); Melrose Highlands (Clemons); Cambridge (Henshaw); Essex Co., Andover (Hagen); Waltham (Lathrop); Wollaston (Sprague); Southbridge (Bromley).

Rhode Island: Providence (Davis); Buttonwoods (Johnson).

Connecticut: Litchfield? (Woodruff); New London (Osburn).

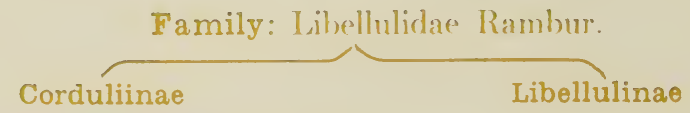

largetubercle in Macromiini

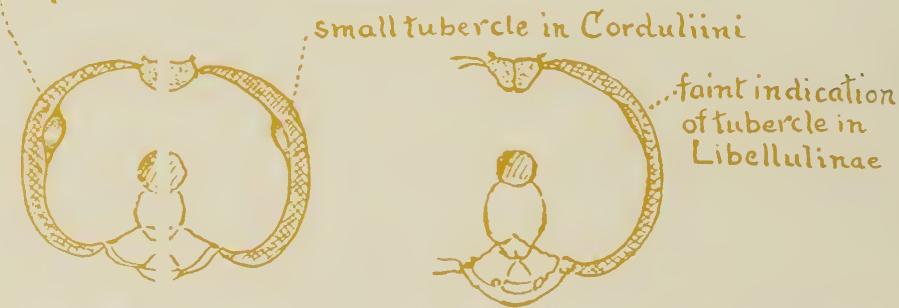

Hind margin of eye bulged in

form tulercle.

Hind margin of eye not bulged to form tuberife (Ser Libellulinae)

Note: 'Ihe above character can best be seen after removal of head. 

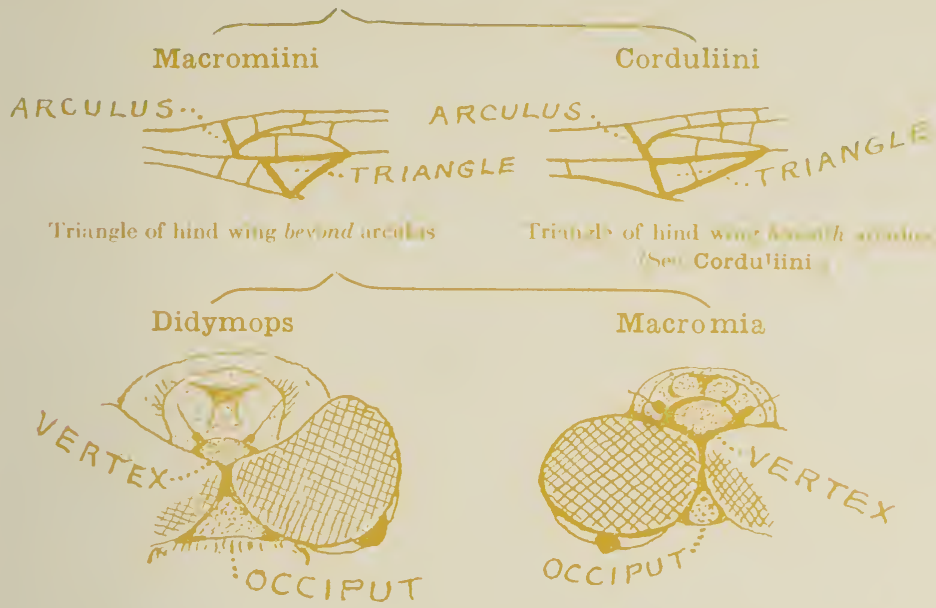

Vertex smaller Ihan occiput.

Tribe: Macromiini Needham.

Genus: Didymops Rambur.

Me. N. H. Vt. Mass. R. I. Conn.

Didymops transversa . . . . ++++

95. Didymops transversa (Say).

Journ. acad. nat. sci. Phila. 8: 19. 1839. Type locality: "Massachusetts."

Common,- brooks, rivers, ponds, lakes.

Nay 13 to August.

Maine: Foxcroft, So. Lagrange, Rangeley, Orono (Harvey, Cabot); Manchester (Wadsworth); Capens (Johnson).

-New Hampshire: Manchester (Burnham); Exeter, Meredith Neck (Howe); Squam Lake, Jaffrey (Allen); Peterboro (Cabot).

Vermont: (Hagen).

Massachusetts: Salem, Woburn, Worcester (Cabot); Cambridge, Stow, Milton (Hagen); West Bridgewater (Tower); Concord (Howe); So. Natick, Sherborn (Morse); Boston, Rutland, Mt. Tom (Johnson); Winchendon (Russell); Wollaston (Sprague); Wareham (Bangs); Groton (Low); Waverley (Hagen); Nahant (M. C. Z.); Southbridge (Bromley).

Connecticut: Litchfield (Woodruff); New London (Osburn). 
Genus: Macromia Rambur.

Me. N. H. Vt. Mass. R. I. Conn.

Macromia illinoiensis . . . . + ++

96. Macromia illinoiensis Walsh.

Proc. acad. nat. sci. Phila. 397. 1862. Type locality: Rock Island, Illinois.

Uncommon,-- larger streams, lakes, roads.

June 18 to August 16.

Maine: Orono, Bradley (Harvey); Manchester (Wadsworth).

New Hampshire: Manchester (Burnham); Meredith Neck, Moultonboro (Howe); Intervale, Squam Lake (Allen).

Massachusetts: Sherborn (Babcock); Worcester (Hitchings); Woods Hole (Hagen); Westport (Howe); Wareham (Bangs).

Rhode Island: Tiverton (Howe).

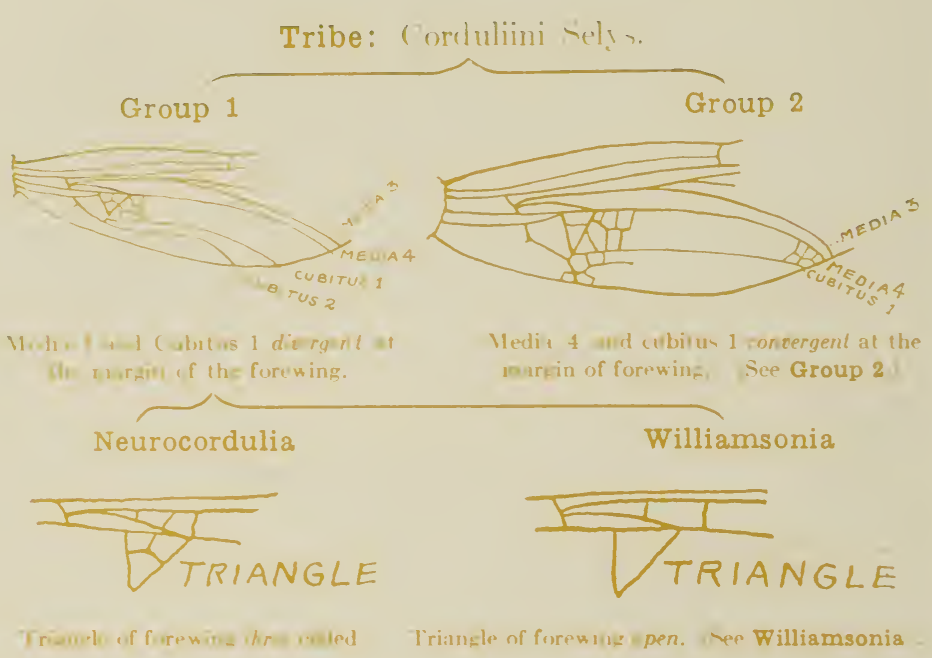

Genus: Neurocordulia Sely's.

Neurocordulia obsoleta . . . + + +

yamaskanensis . + 
97. Neurocordulia obsoleta (Say).

Journ. acad. nat. sci. Phila. 8: 28. 1839. Type locality: "Indiana and Massachusetts."

Rare,- - streams, lakes, crepuscular.

June 10 to August 30 .

Maine: Manchester (Wadsworth).

New Hampshire: Manchester (Burnham); Meredith Neck (Howe).

Massachusetts: Milton (Hagen); Southbridge (Bromley); No. Reading (Johnson).

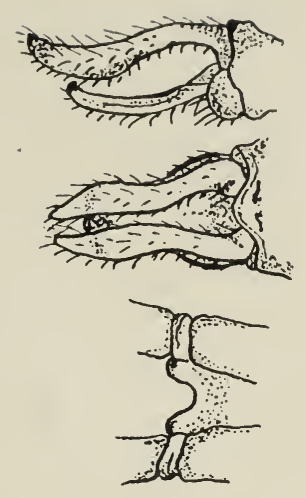

98. Neurocordulia yamaskanensis (Prov.).

Nat. canad. 7: 248. 1875. Type locality: "St. Hyacinthe," Quebec.

Very rare,-lakes, wholly crepuscular. June.

Maine: Orono (Harvey).
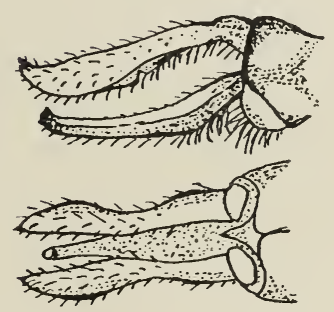

Genus: Williamsonia Davis.

Me. N. H. Vt. Mass. R. I. Conn.

Williamsonia lintneri

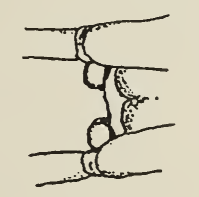

All abdominal segments ringed with yellow.

99. Williamsonia lintneri (Hagen).

N. Y."

Bull. acad. Belg. ser. 2, 45: 187. 1878. Type locality: "Centre,

Rare,- ponds.

April 30 to June 1.

Massachusetts: Concord (Peirson, Howe); Dedham (Johnson); Blue Hills (Clench); Sherborn (Smith). 


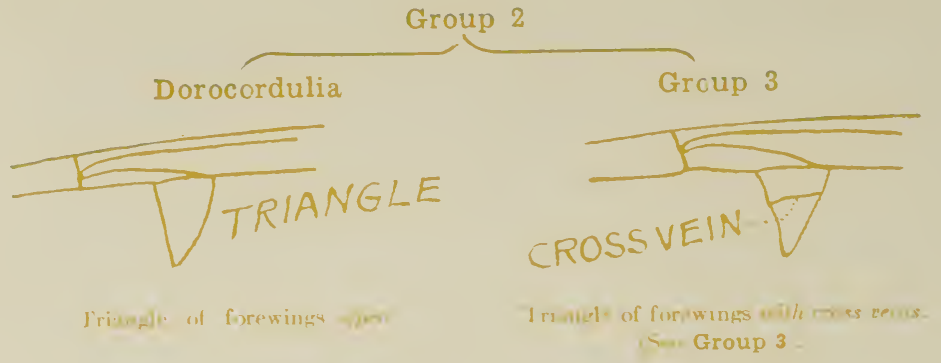

Genus: Dorocordulia Needham.

Me. N. H. Vt. Mass. R. I. Conn.

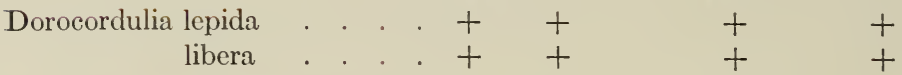

100. Dorocordulia lepida (Hagen).

Bull. acad. Belg. 31: 264. 1871. Type locality: "Massachusetts,"

- New York, Albany,- Maryland,- New Jersey."

Uncommon,- ponds.

May 22 to August 31.

Maine: Greenfield, Orono, Bradley (Harvey); Manchester (Wadsworth);

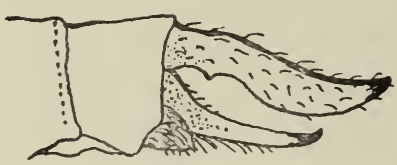
Portland (Hagen).

New Hampshire: Manchester (Burnham); White Mts. (Morrison).

Massachusetts: Cambridge, Stow, Natick, Brookline (Hagen); Sherborn (Babcock); West Bridgewater (Tower); Woods Hole (Kellicott); Concord (Howe); Blue Hills (Johnson); Wareham (Bangs); Waltham (Bullard); Magnolia, Brookline (Hagen); Newton (Hubbard).

Rhode Island: Wickford (Johnson).

Connecticut: Litchfield (Woodruff); Hammond's pond (Hagen).*

* This locality is probably Brookline, Mass., where there is a well known pond by this name.

101. Dorocordulia libera (Selys).

Bull. acad. Belg. 31: 236. 1871. Type locality: "Canada."

Uncommon,- ponds, lakes.

June $S$ to August.

Maine: Greenfield, Orono (Harvey); Manchester (Wadsworth).

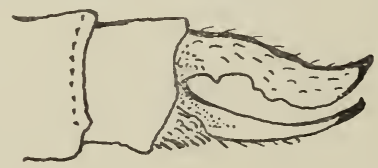


New Hampshire: Franconia (Slosson); Moultonboro (Howe); Jaffrey (Johnson); Peterboro (Cabot).

Massachusetts: Sutherland (Dwyer); Concord (Howe); West Groton (Lahee).

Connecticut: Litchfield (Woodruff); Union (Bromley).

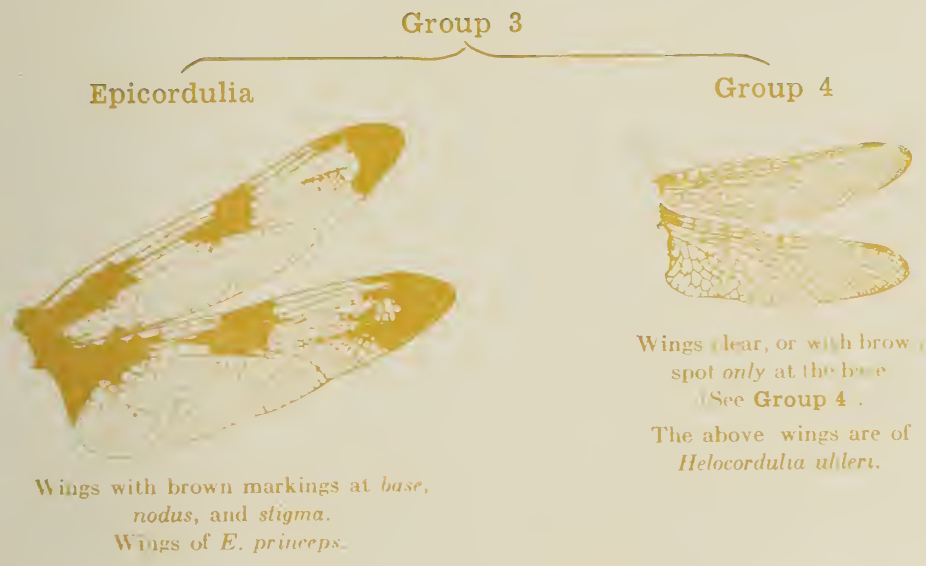

Genus: Epicordulia Selys.

Me. N. H. Vt. Mass. R. I. Conn.

Epicordulia princeps . . . . ++++++

\section{Epicordulia princeps (Hagen).}

Syn. Neur. No. Amer. 134. 1861. Type locality: "Pecos River, Western Texas; Georgia; Maryland."

Common,- - ponds, lakes, uplands.

May 25 to August 27.

Maine: Manchester (Wadsworth).

New Hampshire: Meredith Neck, Moultonboro, Concord (Howe).

Massachusetts: Cambridge (Cabot); Natick, Sherborn (Babcock); Worcester (Hitchings); Concord (Howe); Fall River (Holt); Cataumet (Winsor); Wellesley (Morse); Webster (Bromley); Wareham (Bangs).

Rhode Island: Tiverton (Howe).

Connecticut: New Haven (Hagen); Litchfield (Woodruff); New London (Osburn). 
Helocordulia
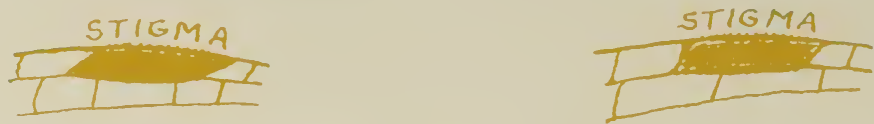

Slemat demond shaped

- lizma almost reclangular.

See Group 5).

Note: The form of the stigma is sometimes misleading, but an error made here is quickly apparent in following out the characters for the following genera. A cross vein in one of the forewings is sometimes absent, very rarely in both wings. The brown spot at the base of all wings in such unusual cases will serve to distinguish the species from the two foregoing species of Dorocordulia.

Genus: Helocordulia Needham.

Helocordulia uhleri

Me. N. H. Vt. Mass. R. I. Conn.

\section{Helocordulia uhleri (Selys).}

Bull. acad. Belg. ser. 2. 31: 274. 1871. Type locality: "Randal, Maine.... New Jersey."

Uncommon,- ponds, rivers, upland woodlands.

May 15 to July 12.

Maine: Orono (Harvey); Manchester

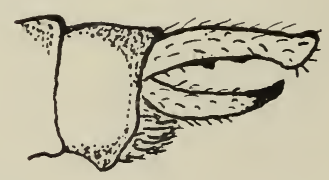
(Wadsworth); Echo Lake, Mt. Desert Johnson).

New Hampshire: Franconia (Howe).

Massachusetts: Stow (Hagen); West Bridgewater (Tower); Concord (Howe); Fall River (Holt); Amherst (B. S. N. H.).

Connecticut: Litchfield (Woodruff).
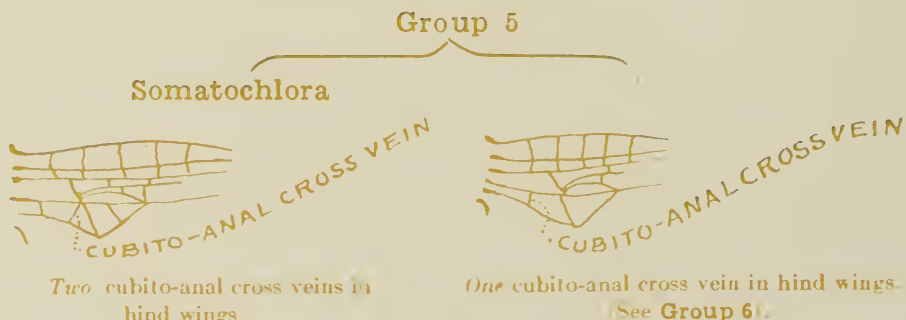

fre cubilo-anal cross vein in hind wings see Group 6 . 
Genus: Somatochlora Selys.

Me. N. H. Vt. Mass. R. I. Conn.

Somatochlora albicincta

cingulata

$+$

elongata . . ++

forcipata . . . ++

franklini . . . +

kennedyi . . . ++

linearis . . . . +

minor . . . + +

tenebrosa . . +++

walshii . . . ++

$+$

williamsoni

A. All segments of abdomen ringed with white.

1. Abdomen under $32-34 \mathrm{~mm}$. long.

104. Somatochlora albicincta (Burm.).

Handb. ent. 2: 847. 1839. Type locality: "Labrador."

Uncommon,- - ponds, lakes.

July 4 to August 11.

New Hampshire: Hermit Lake, Mt. Washington (Scudder); Waterville (Hagen); Carter Notch (Allen); Lonesome Lake, Profile Lake (Howe).

2. Abdomen over $40-41 \mathrm{~mm}$. long.

\section{Somatochlora cingulata}

$$
\text { (Selys). }
$$

Bull. acad. belg. 31: 302. 1871. Type locality: "Terre-Neuve."

\section{Rare,-- lakes.}

New Hampshire: White Mts. (Hagen); Carter Notch (Allen).

B. Few or no segments of abdomen ringed with white.

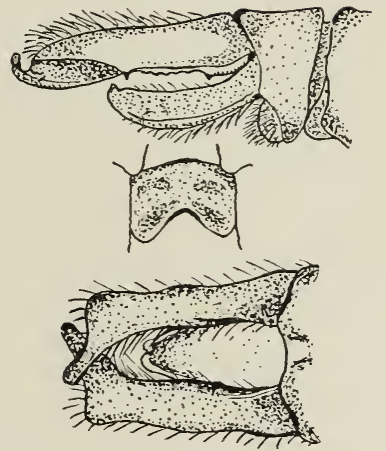

Side view of male abdominal appendages. Vulvar of female. Male abdominal appendages from above.

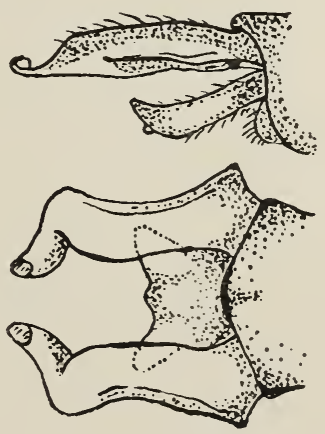

1. Abdomen over $40 \mathrm{~mm}$. long.

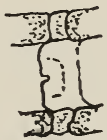


106. Somatochlora elongata (Scudder).

Proc. Bost. soc. nat. hist. 10: 218. 1866. Type locality: "White Mts."

Uncommon,- lakes.

July 8 to August 12.

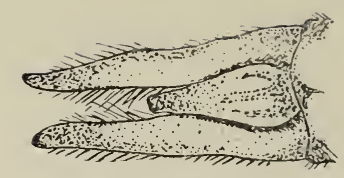

Maine: at sea off Scoodic point (Harvey); Bradley (Harvey).

New Hampshire: Fabyan's, Hermit Lake, Mt. Washington (Scudder, Calvert); Franconia (Slosson); Ply-
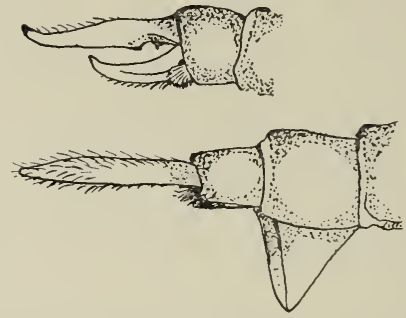
mouth (Hagen); Centre Harbor, Jackson (Howe); Bretton Woods (Woodruff).

\section{Somatochlora linearis}

(Hagen).

Syn. Neur. No. Amer. 137. 1861. Type locality: "St. Louis."

Very rare,- - even doubtful. July 27 .

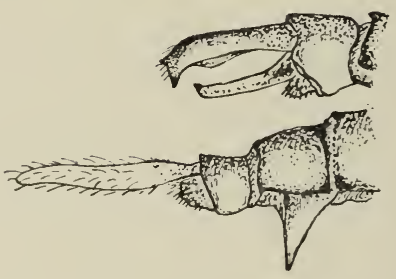

Maine: Manchester (Wadsworth).

108. Somatochlora williamsoni Walker.

Can. Ent. 39: 69. 1907. Type locality: "Toronto," etc.

Very rare,- lakes.

July 17.

New Hampshire: Meredith Neck

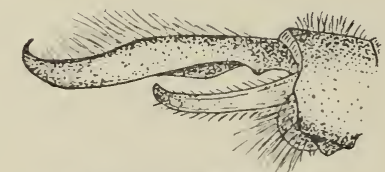
(Howe).

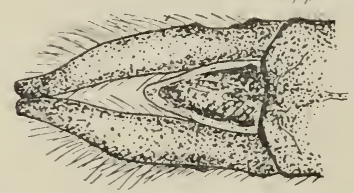

2. Abdomen under $40 \mathrm{~mm}$. long. 
109. Somatochlora forcipata (Scudder).

Proc. Bost. soc. nat. hist. 10: 216. 1866. Type locality: "The Glen, White Mts."

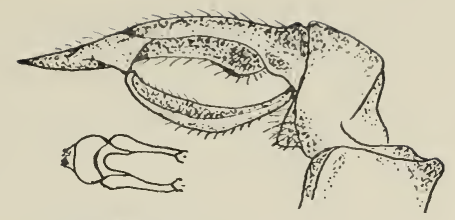

Uncommon,- - lakes, ponds.

May to August.

Maine: Orono, Bradley (Har-

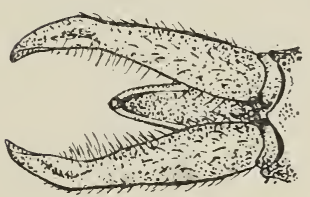
vey); Manchester* (Wadsworth).

New Hampshire: Mt. Washington (Slosson); The Glen, White Mts. (Scudder); Profile Lake (Howe).

*A doubtín record.

\section{Somatochlora kennedyi \\ Walker.}

Can. Ent. 50: 371. 1918. Type locality: "Orono, Me."

Uncommon,- - ponds, lakes, uplands.

June 3 to July 16.

Maine: Orono (Harvey); Man-
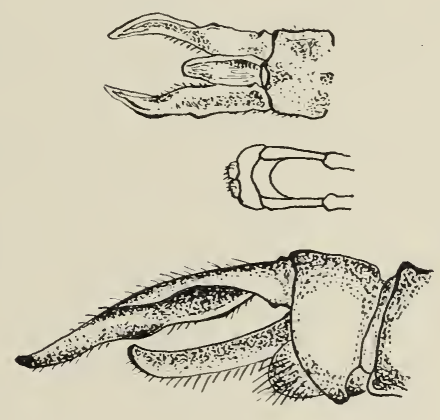
chester (Wadsworth); Capens (Johnson).

New Hampshire: Boscaween (Howe).

Massachusetts: Concord (Howe).

111. Somatochlora minor (Calv.).

Ent. News 9: 87. 1898. Type locality: "Bradley, Maine; Franconia, N. H."

Rare,

June 22 to July 8.

Maine: Bradley (Harvey).

New Hampshire: Franconia (Slosson).
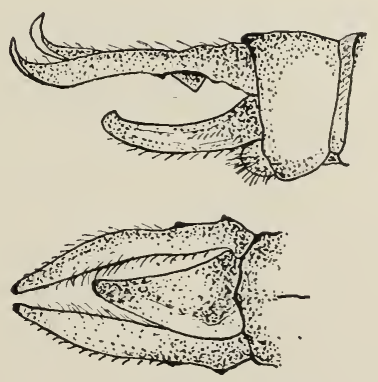

Tip of superior appendages usually less upcurved 
Rhode Island: Bristol? (Howe).

Connecticut: Litchfield (Troodruff).

\section{Somatochlora walshii (Scudder).}

Proc. Bost. soc. nat. hist. 10: 217. 1566. Type locality: "The Glen, White Mts."

Rare,- rivers.

June to September 15.

Maine: Orono, Bradley (Harver);

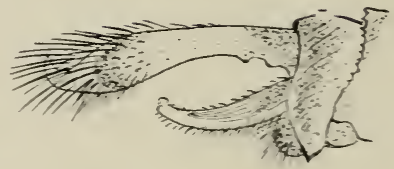

Manchester (Wadsworth).

New Hampshire: The Glen, Thite Mts. (Scudder).

Massachusetts: Concord (Howe).

3. Abdomen tariable $33-4 \mathbf{~ m m}$. long.

\section{Somatochlora franklini}

$$
\text { (Selys).* }
$$

Bull. acad. Belg. ser. 2. 45: 195. 1575. Type locality: "Labrador."

Very rare,-

June $1 \mathrm{~s}$.

Maine: Chemo stream, Orono (Harrey).

* = Somatochlora septentrionalis (Hagen).
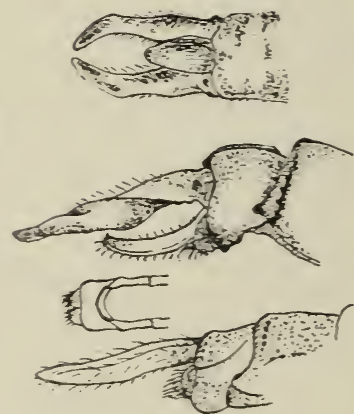

\section{Somatochlora tenebrosa (Say).}

Bull. acad. Belg. ser. 2. 31: 2\$9. 1 \$71. Type locality: "Indiana."

Uncommon,- brooks.

August 1 to September $s$.

New Hampshire: Moat Mt., North Conway (Calvert); Intervale (Allen).

Massachusetts: Concord (Howe); Southbridge (Bromler); Wareham (Bangs).
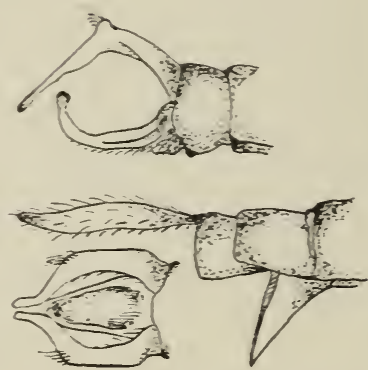


\section{$\overbrace{\text { Cordulia }}^{\text {Group } 6} \overbrace{\text { Tetragoneuria }}^{\text {Geta }}$}
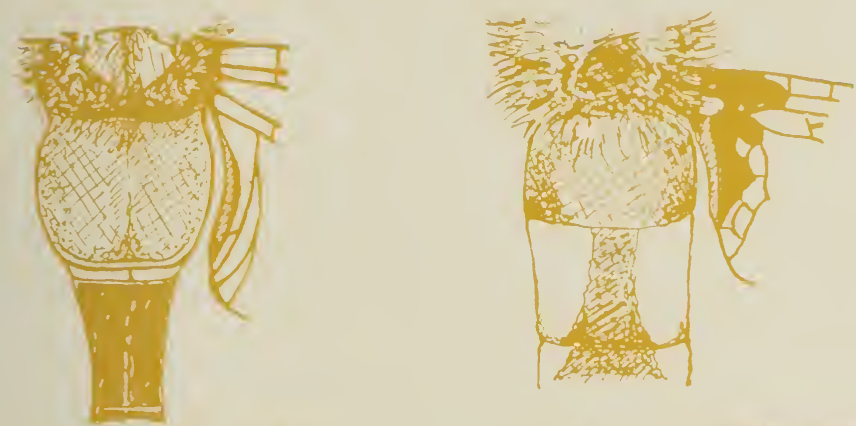

First and second untervibiti wil.

Thund wings withoul dirk markm=

lined athdominal segment on

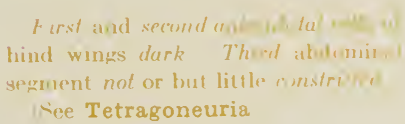

Genus: Cordulia Leach.

Me. N. H. Vt. Mass. R. I. Conn.

Cordulia shurtleffi

$+++$

\section{Cordulia shurtleffi Scudder.}

Proc. Bost. soc. nat. hist. 10: 217. 1866. Type locality: "Hermit Lake." Mt. Washington, N. H.

Common,-- ponds, lakes.

May 30 to August 11.

Maine: Orono, Bradley (Harvey).

New Hampshire: Hermit Lake, Mt.

Washington (Scudder, Calvert); Craw- The forked (side view) lower ap-

ford Notch (Slosson); Moultonboro, pendage in the male is diagnostic. Echo Lake (Howe); Intervale (Allen); Peterboro (Cabot).

Massachusetts: Concord, Wayland (Howe); Mt. Greylock (Calvert); Framingham (Frost).

Connecticut: Litchfield (Woodruff).

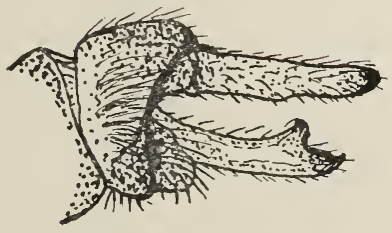


Genus: Tetragoneuria Hagen.

Me. N. H. Vt. Mass. R. I. Conn.

Tetragoneuria canis

costalis

cynosura . . ++ ?

cynosura simulans ++

morio . . . . +

spinigera . . . ++

$\begin{array}{ll}+ & + \\ + & + \\ + & \end{array}$

A. Hind wings 24 to $31 \mathrm{~mm}$. long. Rarely with brown T-spot on top of frons clearly developed. Male abdominal appendages from above widely divergent. Female abdominal appendages not exceeding $1.7 \mathrm{~mm}$. long.

a. Brown of hind wing not reaching triangle.

\section{Tetragoneuria cynosura (Say).}

Journ. acad. nat. sci. Phila. 8: 30. 1839. Type locality: "Massachusetts."

Common, - brooks, ponds, lakes, uplands.

Nay 3 to July 9.

Maine: Rangeley, Bradley, Orono (Harvey); Manchester (Wadsworth); Andover (M. C. Z.).

New Hampshire: Meredith Neck (Howe).

Massachusetts: Everett, Wakefield, Boston (Cabot); Stow, Brookline, Canton (Hagen); Sherborn (Babcock); Worcester (Hitchings); Rutland, Framingham, Auburndale (Johnson); Concord (Howe); Fall River (Holt) Woods Hole (Osburn); No. Saugus (Titus); Green Lodge, So. Natick, Wellesley (Morse); Wollaston (Sprague); Warat var, and abdominal apNewton (Thaxter); Southbridge (Bromley).

Connecticut: Litchfield (Woodruff); New Canaan (Atwater); Danbury (Johnson); New London (Osburn).

b. Brown of hind wings reaching to or including triangle.

\section{Tetragoneuria cynosura simulans Mutt.} Ind."

Bull. Wis. nat. hist. soc. 9: 196. 1911. Type locality: "Bluffton 
Uncommon,- ponds, lakes, brooks, woodlands.

April to July 20.

Maine: Bradley (Harvey); Mt. Desert (Bullock); Manchester (Wadsworth); Echo

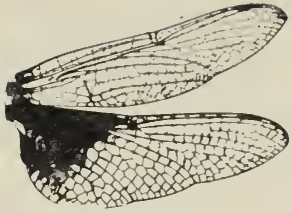

Wings of T. c. simulans.

Lake, Mt. Desert, Calais (Johnston); Andover (M. C. Z.).

New Hampshire: Manchester (Burnham); Meredith Neck, Moultonboro (Howe); Fitzwilliam (Barnes); Jaffrey (Johnson); Squam Lake (Allen); Peterboro (Cabot).

Massachusetts; Worcester (Hitchings); Martha's Vineyard (Moore); Concord (Howe); Blue Hills (Clench); Sherborn (Morse); Southbridge (Bromley); Waltham (Bullard); Wareham (Bangs); Framingham (Johnson); Brookline, Waverley (Hagen).

Connecticut: Litchfield (Woodruff).

Note:-T. c. semiquea Burm. of Dr. Calvert's list is a southern species whose range is restricted to the south. (See Dr. Muttkowski's paper, Bull. Wis. Nat. Hist. Soc. 9:91-134. 1911 and 13:49-61. 1915).

B. Hind wings 29 to $33 \mathrm{~mm}$. long. Brown T-spot on top of frons always clearly present. Male abdominal appendages from above but slightly divergent.

a. Male superior appendages without ventral spine. Female abdominal appendages over $1.7 \mathrm{~mm}$., but under $2 \mathrm{~mm}$. long.

\section{Tetragoneuria morio Mutt.}

Bull. Wis. nat. hist. soc. 9: 125. 1911. Type locality: "Solon Springs, Douglas Co., Wis."

Rare,- ponds, woodlands.

May 18 to June 29.

Maine: (Harvey).

New Hampshire: Meredith Neck (Howe).

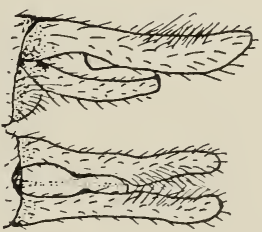

Massachusetts: Concord (Howe); Boston, Framingham (Johnson).

b. Male superior abdominal appendages with ventral spine. Female abdominal appendages over $3 \mathrm{~mm}$. long.

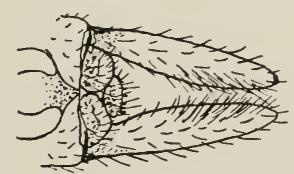




\section{Tetragoneuria spinigera (Selys).}

Bull. acad. Belg. ser. 2. 31: 269. 1871. Type locality: "Canada." Uncommon,- - ponds, lakes, woodlands.

May 19 to July 14.

Maine: Bradley (Harvey); Manchester (Wadsworth).

New Hampshire: Manchester (Burnham); Peterboro (Cabot); Meredith Neck (Howe); Squam Lake (Allen).

Massachusetts: Winchendon (Morse); Concord (Howe); Groton (Low).

Connecticut: Litchfield (Woodruff).

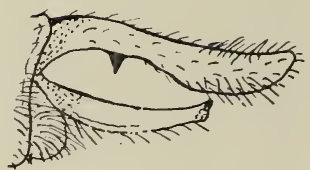

C. Hind wings 30 to $32 \mathrm{~mm}$. long. Brown T-spot on top of frons never present.

b. Male superior abdominal appendages with tubercle above. Female abdominal appendages only $3 \mathrm{~mm}$. long.

\section{Tetragoneuria canis MacLach.}

Ent. Mo. Mag. 23: 104. 1886. Type locality: "Washington Territory."

Very rare, - ponds.

June 18 .

New Hampshire: Jaffrey (Johnson); Franconia (Slosson).
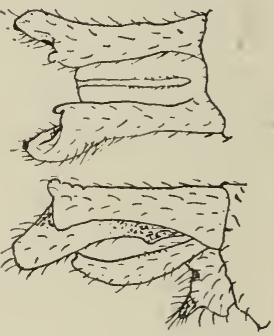

\section{1?. Tetragoneuria costalis Selys.}

Bull. acad. Belg. ser. 2. 31: 273. 1871. Type locality: "Georgie americaine."

One record.

June 9, 1898.

Maine: Manchester (Wadsworth in U. S. N. M.).

Note: $T$. costalis is of doubtful status as a species, and the record of $T$. spinosa from Franconia, N. H., of Dr. Calvert and most authors should be referred to T. canis (see Dr. Muttkowski's paper). T. spinosa is a southern species and very distinct. 


\section{Additional Stations}

\section{Agrion amatum}

Pant I

June 8

Agrion aequabile

Vermont: Brandon, Leicester (Dutton).

Agrion maculatum

Vermont: Bristol (Dutton).

Massachusetts; Wareham (Bangs); Groton (Low).

Lestes congener

Massachusetts: Weston (M. C. Z.).

Rhode Island: Bristol (Howe).

Lestes eurinus

Massachusetts: Wareham (Bangs).

Lestes unguiculatus

September 6

Massachusetts: Wareham (Bangs).

Vermont: So. Hero, Grand Isle (Morse).

Lestes uncatus

Vermont: So. Hero (Morse).

Lestes disjunctus

\section{Part II}

Vermont: Woodstock (Morse).

Massachusetts: Wellesley (Morse).

Lestes rectangularis

Massachusetts: Wareham (Bangs).

Lestes vigilax

Massachusetts: Lynnfield (Little).

Lestes inequalis

September 19

Massachusetts: Heath (Warden); Wareham (Bangs).

Argia moesta

Massachusetts: Wellesley (Morse).

Argia violacea

Massachusetts: Sherborn, Wellesley (Morse); West Peabody (Little).

Enallagma durum

June

Massachusetts: Wareham (Bangs).

Enallagma calverti

May 12

Enallagma ebrium

Vermont: So. Hero, Woodstock (Morse): Bristol (Dutton).

Massachusetts: Wakefield (Atwater); Lynnfield (Little).

Enallagma traviatum

July 8

Massachusetts: Wareham (Bangs).

Enallagma aspersus

September 6

Rhode Island: Bristol (Howe).

Enallagma civile

September 22

Lynnfield (Little).

Enallagma pictum

July 8

Massachusetts: Wareham (Bangs).

\section{Enallagma geminatum}

October 2

Enallagma signatum

Massachusetts: Wareham (Bangs).

Enallagma pollutum

Massachusetts: Wakefield (Atwater). 


\section{Nehalennia irene}

Vermont: So. Hero, Woodstock (Morse).

Massachusetts: Wakefield (Atwater).

\section{Amphiagrion saucium}

July 29.

Vermont Bristcl (Dutton).

Massachusetts: Wareham (Bangs).

Chromagrion conditum

Vermont: Bristol (Dutton).

Ischnura verticalis

Vermont: So. Hero, Woodstock (Morse): Bristol (Dutton).

Massachusetts: Wakefield (Atwater); Wareham (Bangs).

\section{Ischnura posita}

September 18

Massachusetts: Wakefield (Atwater).

\section{Anomalagrion hastatum}

Massachusetts: Wareham (Bangs).

\section{Part III}

Cordulegaster diastatops

Massachusetts: Wareham (Bangs).

Connecticut: (Norton).

Ophiogomphus rupinsulensis

New Hampshire; Franconia (Slosson).

Ophiogomphus aspersu3

Vermont: Brandon (Dutton).

Gomphus albistylus

Massachusetts: Wellesley (Morse).

Gomphus abbreviatus

New Hampshire; Franconia (Slosson).

Gomphus brevis

Vermont: Brandon (Dutton).

\section{Gomphus exilis}

New Hampshire: Squam lake (Allen).

Vermont: Bristol (Dutton).

Massachusetts: Mt. Wachusett (Sprague).

Gomphus furcifer

Massachusetts: Bedford (Henshaw).

\section{Gomphus spiniceps}

June

Hagenius brevistylus

Massachusetts: Wareham (Bangs).

\section{Recapitulation of New England Aeschnidae and Libellulidae (Cordulinae)}

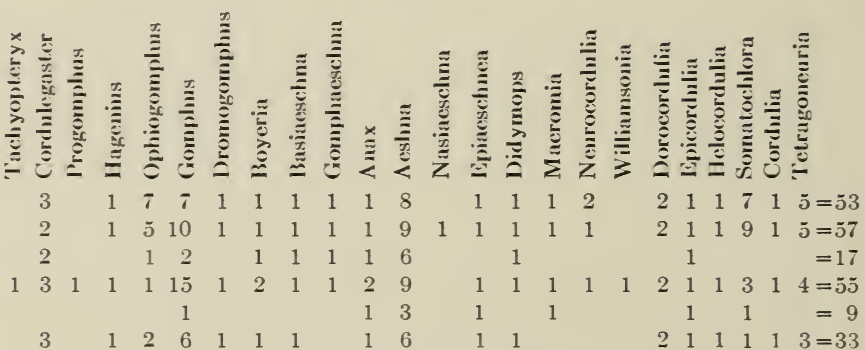






\section{Memoir of the Thoreau Museum of Natural History: II}

\section{Manual of the Odonata of New England}

BY R. HEBER HOWE, Jr.

\section{Part V.}

January 8, 1920.

Subfamily: Libellulinae Selys.

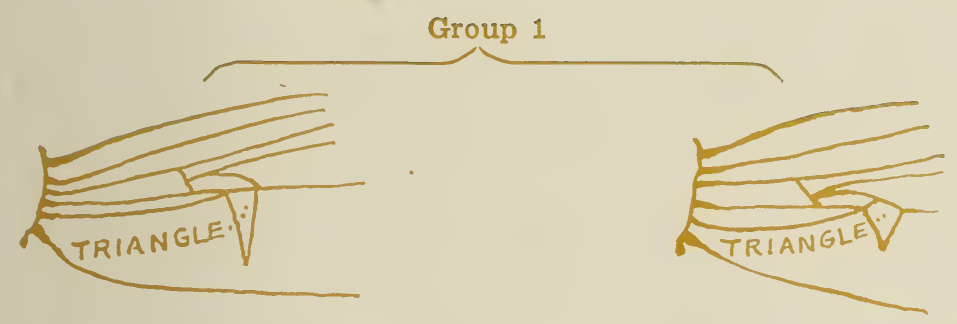

Triangle of fore-wings typical,three sided.

Triangle of fore-wings at ypical. four sided (See Nannothemis
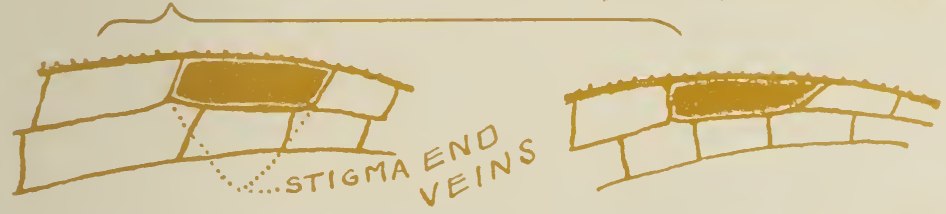

Stigma with end veins parallel.

Stigma with end veins not parallel.

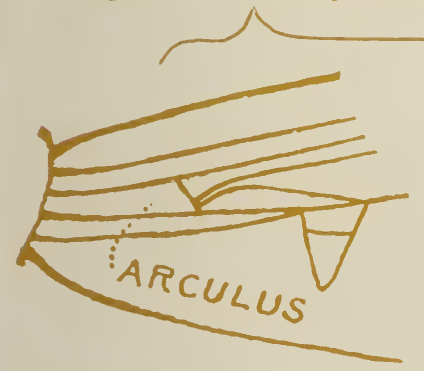

Group 8-Tramea and Pantala

Sectors of the arculus in fore-wings contiguous, but not completely fused beyond the arculus.

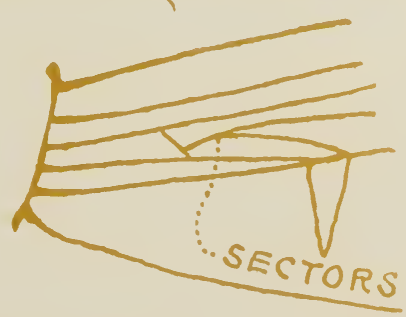

Sectors of arculus in fore-wings fused for short distance $(2-3 \mathrm{~mm}$.) beyond the arculus (See Group 5 


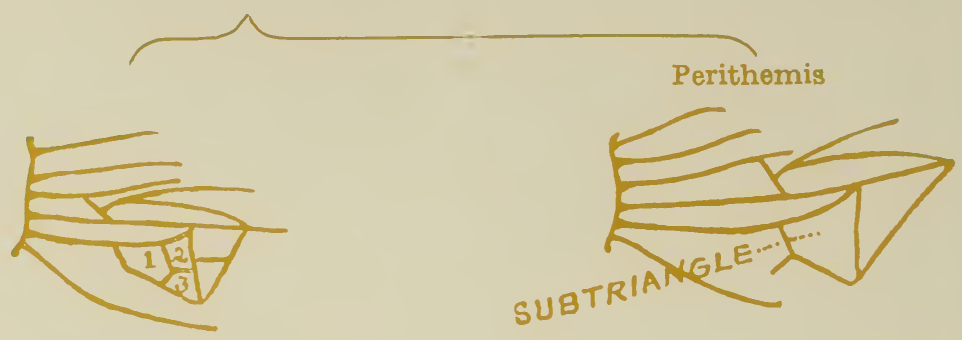

Subtriangle (internal) of fore-wing with three to eleven cells.

Subtriangle (internal) of fore-wings open

\section{Libellula}

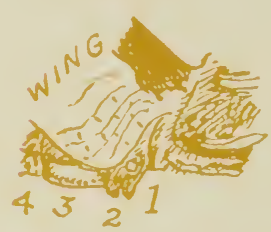

Male with no ventral hooks on segment 1 of abdomen.

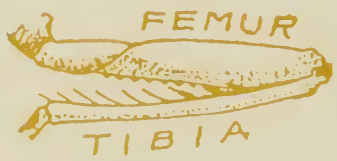

Fimale with tibia of third leg longer than its femur.

\begin{abstract}
(See Perithemis).
\end{abstract}

\section{Plathemis}

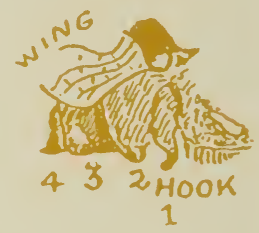

Male with pair of ventral hooks on segment 1 of abdomen.

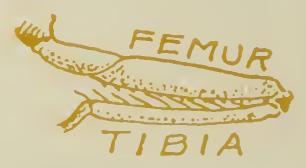

Female with tibia of third leg only as long as its femur (See Plathemis).

\section{Genus: Libellula Linne}

Libellula auripennis . . . . .

Me. N. H. Vt. Mass. R. I. Conn.

cyanea. . . . .

exusta . . . . . + +

flavida . . . .

incesta . . . . . + +

luctuosa . . . .

pulchella . . . . . +

quadrimaculata . . . +

semifasciata . . . . + + vibrans.

$\begin{array}{lllll} & & + & + & + \\ + & & + & & \\ + & + & + & & + \\ & & + & & \\ + & & + & + & + \\ & + & + & + & + \\ + & + & + & + & + \\ + & + & + & & + \\ + & & + & + & + \\ & & + & & +\end{array}$

A. Wings unspotted, suffused with gold, stigma orange. 


\section{Libellula auripennis Burm.}

Handb. ent. 2: 861. 1839. Type locality: "Savannah".

Common,- ponds, lakes,- maritime.

July 4 to August 28.

Massachusetts: Provincetown (Sanborn); Woods Hole, Cuttyhunk (Calvert); Martha's Vineyard (Moore); Nonamesset Island (Osburn); South Orleans, Nantucket (Howe); West Chop (Morse); Wareham (Bangs); Boston (Morrison).

Rhode Island: Watch Hill,

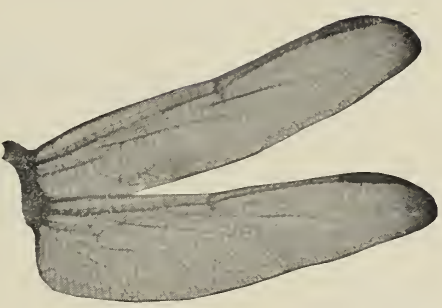

Wings (nat. size). Block Island (Calvert); Middletown (Howe).

Connecticut: Niantic (Morse); Fairfield Co., (Benedict); New London (Osburn).

B. Wings clear, stigma unicolored, black.

(female wings slightly clouded at tips).

\section{Libellula incesta Hagen.}

Syn. neur. 155. 1861. Type locality: "Carolina."

Common,- ponds, lakes.

June 6 to September 17.

Maine: Manchester (Wadsworth).

New Hampshire: Manchester (Burnham); Meredith (Calvert).

Massachusetts: Saugus, Milton (Hagen); Sherborn (Babcock); Worcester (Hitchings); West Bridgewater

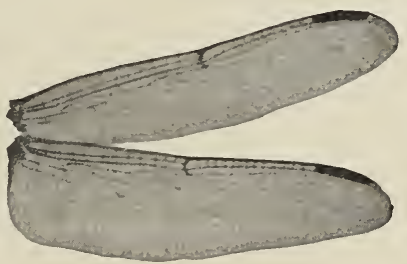
(Tower); Wellesley, Dover, Medfield (Morse); Concord, South Orleans (Howe); Holland (Bromley); Wareham (Bangs); Sharon (Atwater); Framingham (Warden); Bedford (Swett); Brookline (Minot); Southbridge, Webster (Bromley); Woods Hole (Osburn).

Rhode Island: Washington (Johnson).

Connecticut: Danbury (Johnson).

C. Wings with small dark streak at base, and wing tip brownish.

a. stigma unicolored,- black. 


\title{
124. Libellula vibrans Fabr.
}

Syst. ent. 424. 1775. Type locality: "America."

\author{
Rare,- \\ July
}

Massachusetts: Woods Hole (Kellicott, Benedict, Osburn); Fall River (Holt).

Connecticut: S a che m Head (Calvert).

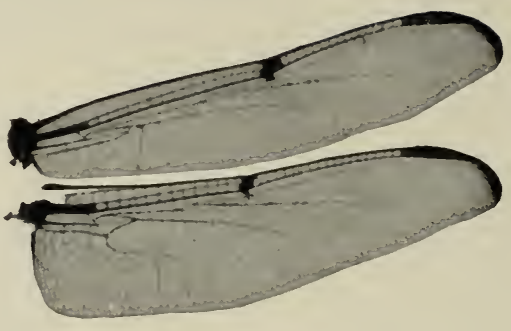

b. stigma, bicolored-black and yellow.

1. Proximal three fourths of stigma yellow.

\section{Libellula flavida Ramb.}

Neur. 58. 1842. Type loculity: not given.

Rare,-ponds.

June 24 to Angust 7 .

Massachusetts: Concord (Howe); Wareham (Bangs).

Note: The determintion of the specimen from Concord recorded above was male by Mr. Williamson. From the series of specimens from Wareham, Mass., collected by Mr. O. Bangs and now in the Museum of Comparative 7oölogy in Cambridge, Mass., Mr. Banks and I were able to select two males and three females larger than the others, with four cells in the interior triangle (should be five) of the fore wings, and with three fourths of the stigma yellow. These specimens represent flavida of Rambur as distinguished by Dr. I'. I'. Calvert. The remaining smaller specimens, three males and two females, have only the typical three cells in the internal triangle, and one half the stigma yellow. It seens evident that flavida represents simply a variation in cyanea, a variation that occurs in individuals, and one that is duplicated in extent in many other species not recognized by name.

2. Proximal half of stigma yellow.

\section{Libellula cyanea Fabr.}

Syrst. ent. 424. 1775. Type locality: "America."

Uncommon,- ponds.

May 24 to September 4.

New Hampshire: Manchester

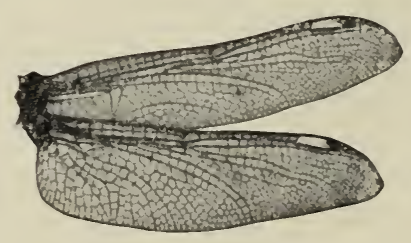
(Burnham). 
Massachusetts: Beverley, Woburn, Cambridge, Natick, Milton (Hagen); Worcester (Hitchings); West Bridgewater (Tower); Woods Hole (Kellicott); Fall River (Holt); Wellesley, Winchendon (Morse); Concord (Howe); Southbridge (Bromley); Sharon (Atwater); Boston (Parshley); Bedford (Swett); Winchendon (Russell); Groton (Low); Sherborn (Babcock); Tyngsboro (M. C. Z.); Arlington (Bullard).

D. Wings with small dark spot at base, and wing tips clear.

Stigma unicolored,- black.

\section{Libellula exusta Say.}

Journ. acad. nat. sci. Phila. 8: 29 1839. Type locality: "Massachusetts."

Common, - ponds, lakes.

May 19 to July 28.

Maine: Bradley, Westbrook (Harvey); Manchester (Wadsworth); Norway (Hagen).

New Hampshire: Franconia (Slosson, Howe); Manchester (Burnham);

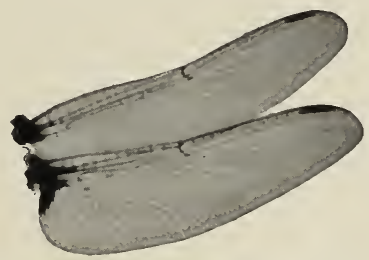
Intervale, Squam Lake (Allen); Moultonboro, Meredith Neck (Howe); Peterboro (Cabot).

Vermont: Brandon, Bristol (Dutton).

Massachusetts: Cambridge, Worcester, Sutton (Hagen, Hitchings); Fall River (Holt); Blue Hills (Clench); Winchendon, Sherborn, Wellesley (Morse); Webster (Bromley); Concord, Chatham, Belmont (Howe); Framingham (Johnson, Frost); Winchendon (Russell); Groton (Low); West Chop (Scudder); West Roxbury (Scudder); Malden (Henshaw); Woods Hole (Osburn).

Rhode Island: Washington (Johnson).

Connecticut: New Haven (Walden); Litchfield (Woodruff); New London (Osburn).

Note: Libellula exusta var. julia (Uhler). Now recognized by Ris as a valid variety, but until the New England status is better known its inclusion in this list will be postponed. L. exusta is said never to have the thoracic dorsum pruinose but all the basal segments so, while in the variety julia the dorsam of the thorax is entirely pruinose, but only the first few basal segments of the abdomen. In exusta the black basal marking on the hind wing includes at least $a$ part of the triangle; in julia it never extends to it. Under this separation I have the following stations for julia : Moultonboro, Franconia, and Meredith, N. H.; Brandon and Bristol, Vt.; Concord, Winchendon, and Framingham, Mass.

E. Wings with large dark basal marking covering one third of wings.

\section{Libellula luctuosa Burm.*}

Handb. ent. 2: 861. 1839. Type locality: "Pennsylvanien."

* = Libellula basalis of earlier writers. 
Uncommon,- ponds.

June 16 to September 6.

Vermont: Brandon, Leiscester (Dutton).

Massachusetts: Concord Brookline, Milton (Howe); West Peabody (Little); Stockbridge (Calvert); Blue

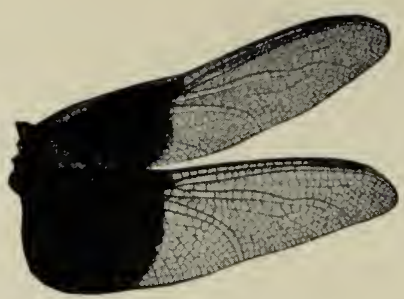

Hills (Clench); Wellesley (Morse); Southbridge (Bromley); Bedford (Swett); New Lenox, Mt. Tom (Johnson); Brookline (Maxcy); Woods Hole (Osburn).

Rhode Island: Wickford (Atwater); Bristol (Howe).

Connecticut: Litchfield (Woodruff); Fairfield Co. (Benedict); New London (Osburn).

F. Wings with black triangular spot at base, and dot at nodus. Front margin suffused with yellow.

\section{Libellula quadrimaculata Linn.}

Syst. nat. 1: 543. 1778. Type locality: "Europa."

Common,-- ponds, lakes.

May 19 to August 15.

Maine: Rangeley, Orono, Bradley

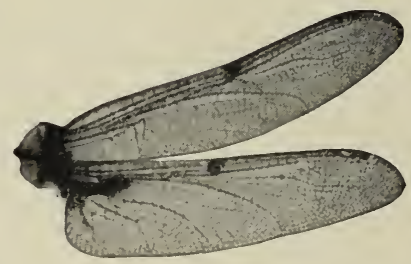
(Harvey); Mt. Desert (Bullock); Manchester (Wadsworth); So. West Harbor (Johnson).

New Hampshire: Franconia (Slosson, Howe); Manchester (Burnham); Meredith Neck, Moultonboro, Lisbon, Lyman, Centre Harbor (Howe); Intervale (Allen); Jaffrey (Johnson).

Vermont: Brandon (Dutton).

Massachusetts: Stow (Hagen); Worcester (Hitchings); Concord (Howe); Green Lodge, Woods Hole (Morse, Osburn); Brookline, Manomet (Johnson); Framingham (Frost); Wareham (Bangs); Southbridge (Bromley).

Connecticut: Sachem Head (Calvert); New Canaan (Atwater); New London (Osburn).

G. Wings with a dark streak at base, a dark spot at nodus, and a dark band across the tip. Front margin suffused with yellow. 
129. Libellula semifasciata Burm.

Handb. ent. 2: 862. 1839. Type locality: "Nord-Amerika."

Uncommon,- ponds.

June 2 to August 16.

Maine: Orono, Bradley, Greenfield (Harvey); Manchester (Wadsworth); Kennebunkport (Woodruff).

New Hampshire: Manchester (Burn-

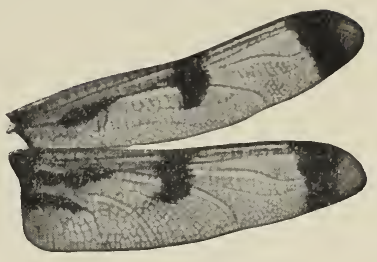
ham).

Massachusetts: Manchester, Cambridge, Stow (Hagen); Woods Hole (Calvert Osburn); Fall River (Holt); Blue Hills (Clench); Concord, Chatham (Howe); Provincetown (Benedict); Dedham, Dover, Green Lodge (Morse); Brookline (Johnson); Martha's Vineyard (Cushman); Wareham (Bangs); Groton (Low); Wollaston (Sprague).

Rhode Island: Bristol (Howe); Buttonwoods (Johnson).

Connecticut: Sachem Head (Calvert); Darien (Johnson); New London (Osburn).

$\mathrm{H}$. Wings with three large dark bands at base, nodus, and near tip, in male with milk white spots between.

Note: The females are apt to be confused with the following species, but the wings in this species are over $41 \mathrm{~mm}$. long, in the next under $33.5 \mathrm{~mm}$. long.

130. Libellula pulchella Drury.

Ill. nat. hist. 1: 115.1770. Type locality: "New York."

Common,- ponds, lakes, and meadow lands.

June 5 to September 8 .

Maine: Orono, Bradley, Auburn, Fryeburg, Westbrook (Harvey); Andover, Brunswick (Hagen); Manchester (Wadsworth).

New Hampshire: Franconia (Slosson); Manchester (Burnham); Centre Harbor, Meredith Neck, Moultonboro, Lisbon, Lyman (Howe); Intervale (Allen); Jaffrey (Johnson).

Vermont: Brandon (Dutton).

Massachusetts: Milton (Hagen); Worcester (Hitchings); Woods Hole, Cuttyhunk, Stockbridge (Calvert); Nantucket, Concord, Brookline, Belmont, Chatham, Orleans, Brewster (Howe); Blue Hills (Clench); 
Fall River (Holt); No. Saugus (Titus); Wellesley, Sherborn (Morse); Southbridge (Bromley); Framingham (Warden); Heath (Parker); New Lenox (Johnson); Wareham (Bangs); Wakefield (Atwater); Cambridge (Osterberg); Arlington, Waltham (Bullard); Wollaston (Sprague); Beverley (Burgess); Forest Hills (Henshəw); Woods Hole (Osburn).

Rhode Island: Providence (Davis); Block Island (Calvert); Tiverton, Middletown (Howe); Bristol (Parker, Clark, Howe); Wickford (Atwater).

Connecticut: Litchfield (Woodruff); Watertown, Burchard's Pond, Fairfield Co. (Benedict); Stanford (Morse); Lyme (Brown); Danbury (Johnson); New London (Osburn); Union (Bromley).

\section{Genus: Plathemis Hagen.}

Plathemis lydia . . . . . . $\begin{gathered}\text { Me. N. H. Vt. Mass. R. I. Conn } \\ ++\end{gathered}$

131. Plathemis lydia (Drury).

Ill. nat. hist. 1: 112. 1770. Type locality: "Virginia."

Common,- brooks, streams, rivers, ponds, lakes.

May 27 to September 19.

Maine: Orono, Bradley (Har-

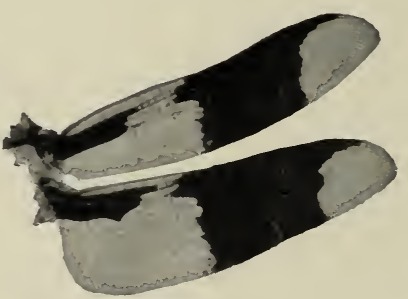

Wings of male. vey); Manchester (Wadsworth); Mt. Desert (Bullock); Bar Harbor (Johnson); Brunswick, Bethel (M. C. Z.).

New Hampshire: Centre Harbor (Howe); Intervale, Squam Lake (Allen); Franconia (Slosson); Manchester (Burnham).

Vermont: Brandon (Dutton).

Massachusetts: Cambridge (Hagen, Atwater); Worcester (Hitchings); Woods Hole, Stockbridge (Calvert); Hingham (Barnes); Cuttyhunk (Ufford); Concord, Brookline, Chatham (Howe); Blue Hills (Clench); No. Saugus (Titus); Southbridge (Bromley); Fall River (Holt); Framingham (Warden); Brookline (Johnson); Ware-

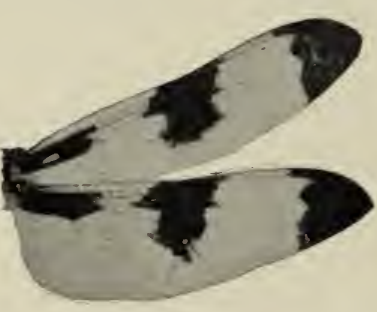

Wings of female. ham (Bangs); Groton (Low); Andover (Scudder); Woods Hole (Osburn); Great Barrington (Johnson). 
Rhode Island: Bristol, Middletown, Tiverton (Howe); Block Island (Calvert); Wickford (Atwater).

Connecticut: Hartford (?); Litchfield (Woodruff); New Canaan (Atwater); No. Guilford (Howe); Darien, Watertown, Burchard's Pond, Fairfield Co. (Benedict); Union (Bromley); New London (Osburn).

\section{Genus: Perithemis Hagen.}

Me. N. H. Vt. Mass. R. I. Conn.

Perithemis domitia tenera.

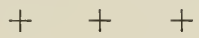

\section{Perithemis domitia tenera (Say).}

Journ. acad. nat. sci. Phila. 8: 31.1839. Type locality: "Indiana, Pennsylvania, and Massachusetts."

Uncommon,- - ponds.

June 12 to September 5 .

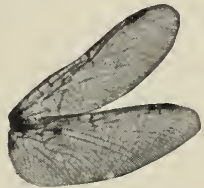

Wings of male (orange).

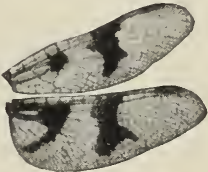

Wings of female.

Massachusetts: Milton (Hagen); Sherborn (Morse, Babcock); Worcester (Hitchings); Fall River (Holt); West Peabody (Little); Concord, Cambridge, South Orleans (Howe); Blue Hills (Clench); Waltham, Wellesley (Morse); Southbridge (Bromley); Sharon, Fall River (Atwater); Bridgewater (Cushman); Swansea (Easton); Bedford (Swett); Brookline (Johnson); Sherborn (Smith); Natick (Stratton): Wareham (Bangs); Holland (Bromley); Woods Hole (Osburn).

Rhode Island: Providence (Davis); Wickford (Atwater); Buttonwoods (Johnson).

Connecticut: Litchfield (Woodruff); Bethlehem, Darien, Burchard's Pond, Fairfield Co. (Benedict); New London (Osburn).

Genus: Nannothemis Brauer.

Me. N. H. Vt. Mass. R. I. Conn. Nannothemis bella . . . . . $++{ }_{+}+$ 133. Nannothemis bella (Uhler.).

Proc. acad. nat. sci. Phila. 87. 1857. Type locality: "Baltimore." Uncommon,- - bogs, ponds. 


\section{June 17 to July 29.}

Maine: Mt. Desert (Bullock); Manchester (Wadsworth).

New Hampshire: Manchester (Burnham); West Swansea (?); No. Conway (Allen); Lyman (Howe).

Massachusetts: Wellesley, Dedham (Morse); (Acad. Nat. Sci., Phila.); Fall River (Holt); Wareham (Bangs); Brewster (Howe); Bridgewater (Cushman); Brookline (Bowditch); Newtonville (Thaxter).

Connecticut: (Hagen).

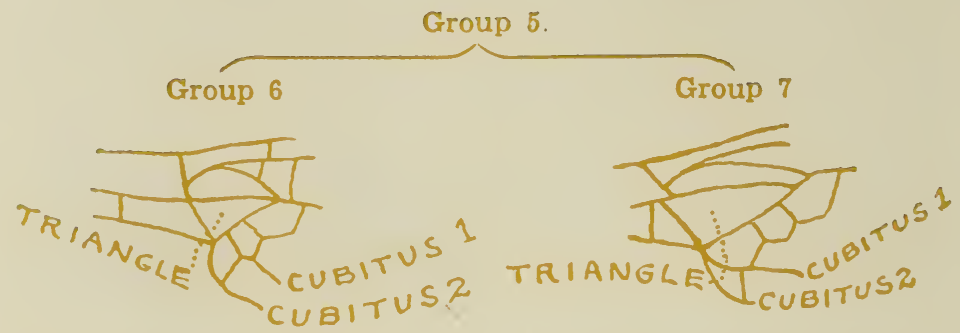

Irin of culutus 1 arising from the ouler side

I pin of cubitus 1 arising from the bollom of the Iriangle in the hind wing. of the triangle in the hind wing. (Ser Group 7 ).

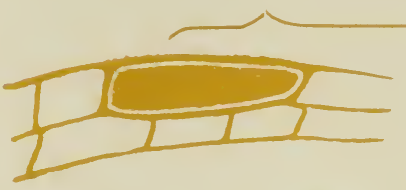

With lwo or more cross velns under stigma.

With one or no cross veiu under stigma. Ser Pachydiplax).
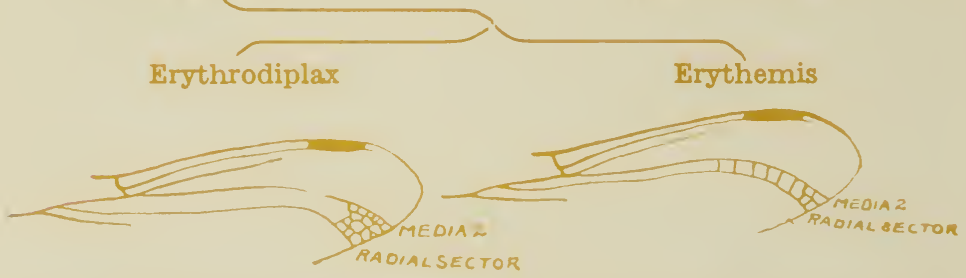

Genus: Erythrodiplax Brauer.

Erythrodiplax berenice Me. N. H. Vt. Mass. R. I. Conn. 


\section{Erythrodiplax berenice (Drury).}

Ill. nat. hist. 1: 114. 1770. Type locality: "Virginia, New York, and Maryland."

Common,- maritime salt marshes.

June 21 to August 14.

Massachusetts: Milton (Hagen); Woods Hole (Calvert, Osburn); Cataumet (Winsor); Nantucket, Chatham, South Chatham, Cotuit (Howe); Hingham (Barnes); Fall River (Holt); So. Dartmouth (Hunt); Faneuil (Morse); Horse Neck Beach, Edgartown, Boston, Eastham (Johnson); Wareham (Bangs); Chelsea Beach (M. C. Z.); Wollaston (Sprague); Revere (M. C. Z.).

Rhode Island: Barrington (Easton).

Connecticut: Niantic, Stamford (Morse); Sachem Head (Calvert); Rowayton (Johnson); Burchard's Pond, Butler's Island, Darien, Fairfield Co. (Benedict); New London (Osburn).

Genus: Erythemis Hagen.

Me. N. H. Vt. Mass. R. I. Conn.

Erythemis simplicicollis . . . +++

135. Erythemis simplicicollis (Say).

Journ. acad. nat. sci. Phila. 8: 28. 1839. Type locality: "Indiana and Massachusetts."

Common,- ponds and rivers.

June 27 to September 6 .

New Hampshire: Manchester (Burnham).

Massachusetts: Natick (Hagen); Worcester (Hitchings); Braintree (Sprague); Woods Hole, Cuttyhunk (Calvert); Concord, Nantucket (Howe); West Peabody (Little); Fall River (Holt); Wellesley (Morse); Provincetown (U. S. N. M., Morse); Framingham (Warden); Bedford (Swett); Forest Hills (Henshaw); Eastham, Auburndale (Johnson); Wareham (Bangs); Newton, Tuckernuck Island (M. C. Z.); Malden (Sprague); Brookline (Scudder); Woods Hole (Osburn); Braintree (Sprague).

Rhode Island: Bristol (Howe); Block Island (Calvert).

Connecticut: Darien, Burchard's Pond, Fairfield Co. (Benedict); Sachem Head (Calvert); Bethlehem (Johnson); New London (Osburn). 
Genus: Pachydiplax Brauer.

Me. N. H. Vt. Mass. R. I. Conn.

Pachydiplax longipennis . . $+++$

\section{Pachydiplax longipennis (Burm.).}

Handb. ent. 2: 850. 1839. Type locality: "Mexico."

Common,-- ponds and rivers.

June 7 to September 9.

Massachusetts: Natick (Hagen); Worcester (Hitchings); West Bridgewater (Tower); Fall River (Holt); West Peabody (Little); Concord, Orleans (Howe); Provincetown, Woods Hole (Benedict, Osburn); Blue Hills, Wellesley (Morse); Sharon (Atwater); Bedford (Swett); Martha's Vineyard (Cushman); Wareham (Bangs); Newton (M. C. Z.); Wollaston (Sprague); Boston (Allen).

Rhode Island: Providence (Davis); Block Island (Allen, Calvert).

Connecticut; Sachem Head (Calvert); Fairfield Co. (Benedict); Darien (Johnson).

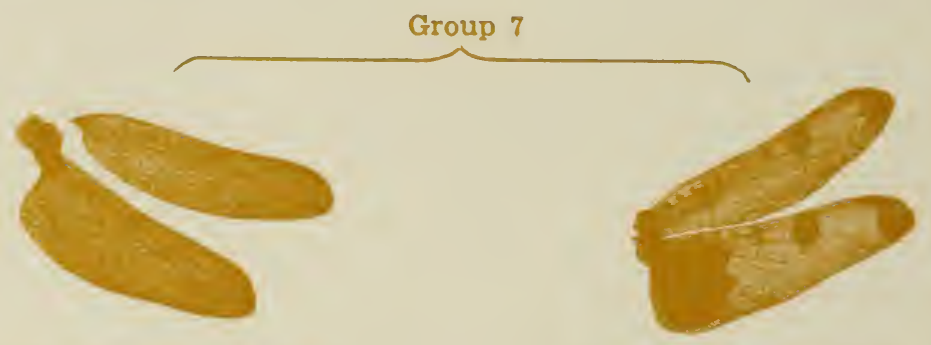

Winks cleur or fainlly suffused with yellow

Wings dark spolled (See Celithemis).

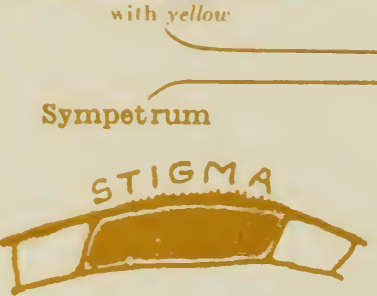

Stigtna thrace as long as wide. 
Genus: Sympetrum Newman.

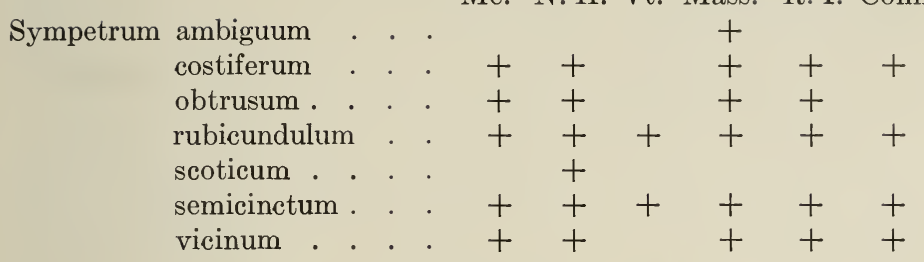

A. Legs black.

a. Wings clear.

1. Wings over $25 \mathrm{~mm}$. long.

\section{Sympetrum rubicundulum (Say).}

Journ.

acad. nat. sci. Phila. 8:

26. 1839 .

Type local-

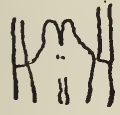

ity: "Indi- Female vulvar ana and lamina. Massachusetts."

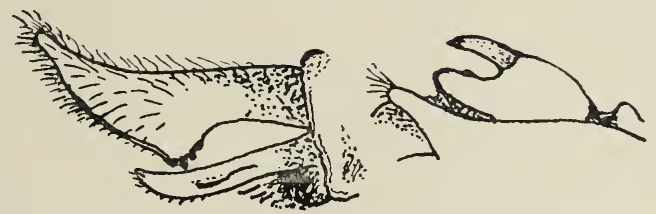

Male abdominal appendages. Male genital hamule. Side view. Side view.

Abundant,- - ponds, lakes.

June 9 to October 9.

Maine: No. West Carry, Mt. Kineo, Rangeley, So. Lagrange, Greenfield, Bradley, Orono, Fryeburg (Harvey); Manchester (Wadsworth); York Harbor (Moore); Echo Lake, Mt. Desert (Johnson).

New Hampshire: Mt. Washington, Hermit Lake, Fabyan's, North Conway (Hagen, Calvert); Franconia (Slosson); Manchester (Burnham); Meredith Neck, Centre Harbor, Moultonboro, Profile Lake, Lisbon, Lyman (Howe); Intervale, North Conway (Allen); Kingston (Morse); Bethelem (Uhler).

Vermont: Newport (Morse); Brandon, Leicester (Dutton); So. Hero (Morse); Rutland (Johnson)

Massachusetts: Worcester (Hitchings); Woods Hole, Cuttyhunk, Mt. Greylock (Calvert); Nantucket (Cockerell, Howe); Concord, (Howe); Kingston (Warden); Hingham (Barnes); West Peabody, Lynnfield (Little); Orleans (Parker); Fall River (Holt); Cotuit (Peirson); Melrose, Highlands (Clemens); Provincetown (Morse, Benedict); No. Saugus (Gowdy); West Chop, Dover, Sherborn, Wellesley (Morse); 
Southbridge (Bromley); Nonquitt (Baldwin); Sharon, Wakefield (Atwater); Framingham (Warden); Edgartown, Auburndale (Johnson); Bridgewater (Cushman); Winchendon (B. S. N. H.); New Bedford (Holcomb); Wareham (Bangs); Natick (M. C. Z.); Brookline (Henshaw); Woods Hole (Osburn).

Rhode Island: Providence (Davis); Block Island (Calvert); Bristol, Middletown (Howe); Wickford (Atwater); Bristol (Clark).

Connecticut: Litchfield (Woodruff); New Canaan (Atwater); Sachem Head (Calvert); Bethlehem (Benedict); Stamford, Deep River, Canaan (Morse); Union (Bromley); New London (Osburn); Lakeville (Calvert).

\section{Sympetrum obtrusum (Hagen).}

Stett. ent. zeit. 28: 95 . 1867. Type locality:"Chicago."

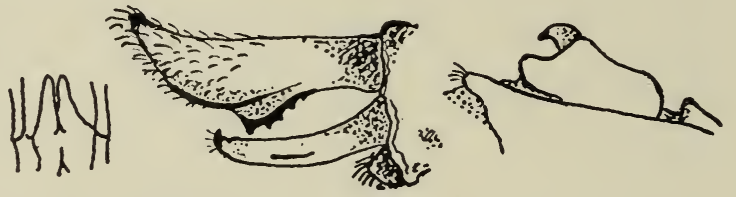

Uncommon,- ponds, lakes.

July 3 to October 5 .

Maine: King \& Bartlett (Laurent); Fryeburg (Harvey); York Harbor (Moore); Mt. Katahdin (U. S. N. M.).

New Hampshire: Fabyan's (Calvert); Meredith Neck, Centre Harbor, Lisbon (Howe); Intervale, North Conway (Allen); Franconia (Slosson).

Massachusetts: Concord, Chatham (Howe); West Peabody (Little); Nonquitt (Baldwin); (Hagen).

Rhode Island: Bristol (Howe).

2. Wings under $23 \mathrm{~mm}$. long

\section{Sympetrum scoticum (Donovan).}

Brit. ins. 15: 523.

1811. Type locality: "bogs of Scotland."

One record,-
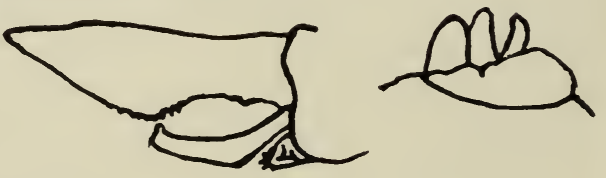

New Hampshire: Franconia (Slosson).

b. Wings with basal half yellow, palest next the body.

1. Wings over $25 \mathrm{~mm}$. long. 
140. Sympetrum semicinctum (Say).

J o u r n.

acad.nat.sci.

Phila. 27.

1839. Type $\bigcap$ if $\bigwedge$

locality: "In-

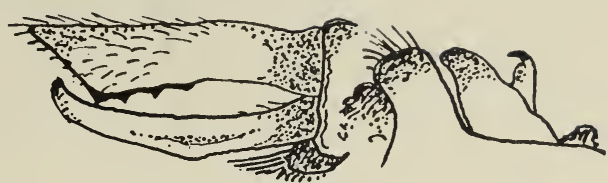

diana and Massachusetts."

Common,- - brooks, ponds.

July 25 to October 5 .

Maine: Orono, Bradley (Harvey); Manchester (Wadsworth); York Harbor (Moore); Ashland Junction (Johnson).

New Hampshire: White Mts. (Hagen); Franconia (Slosson, Howe); Manchester (Burnham); Intervale (Allen); North Conway (Holt, Morse); Fabyan's (Calvert); Lyman (Howe).

Vermont: Brandon (Dutton).

Massachusetts: Milton (Hagen); Worcester (Hitchings); Fall River (Holt); Concord (Howe); Sherborn, Wellesley (Morse); Southbridge (Bromley); Sherborn (Smith); Manomet (Cushman); Framingham (Warden); Wareham (Bangs); Lynnfield (Little); Minot, Saugus (M. C. Z.).

Rhode Island: Wickford (Atwater)

Connecticut: Montville, No. Haven (Morse); New London (Osborn).

c. Wings with front margin yellow, $27 \mathrm{~mm}$. long.

141. Sympetrum costiferum (Hagen).

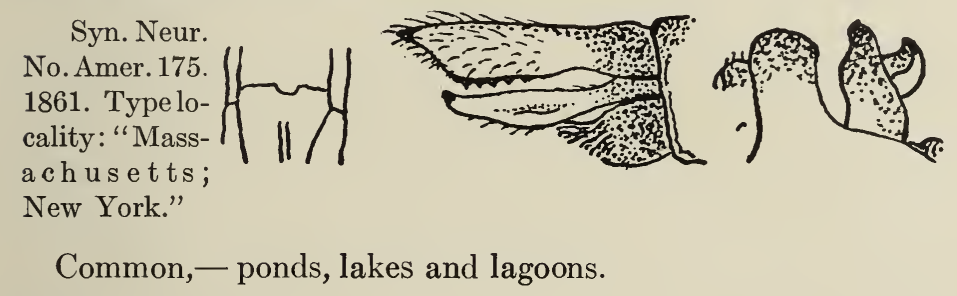

July 12 to October 7.

Maine: Orono, Bradley (Harvey); Manchester (Wadsworth); York Harbor (Moore); Bar Harbor (Johnson).

New Hampshire: Franconia (Slosson, Howe); Intervale, North Conway (Allen); Whitefield (Howe).

Massachusetts: Worcester (Hitchings); Fall River, Pocasset, 
Manomet (Holt); Cuttyhunk (Ufford); Nonamesset Island (Osburn); Provincetown (Benedict); Concord, Nantucket (Howe); Sharon, Fall River (Atwater); Bridgewater, Martha's Vineyard (Cushman); New Bedford (Holcomb); Wareham (Bangs); Lynnfield, West Peabody (Little); Tuckerman Island (M. C. Z.); Wellfleet (M. C. Z.); Brookline (Bowditch); Woods Hole (Calvert).

Rhode Island: Weekopaug (U. S. N. M.); Tiverton (Easton); Watch Hill, Block Island (Calvert).

Connecticut: Litchfield (Woodruff); Bethlehem (Benedict); New London (Osburn).

\section{B. Legs greenish yellow.}

a. Wings $21-26 \mathrm{~mm}$. long.

\section{Sympetrum vicinum (Hagen).}

Syn. Neur. No. Amer. 175. 1861. Type locality: "Bergen Hill, New Jersey; Pennsylvania; Washington."

Common,- brooks, ponds, lakes.

(June 24) August to November 8.
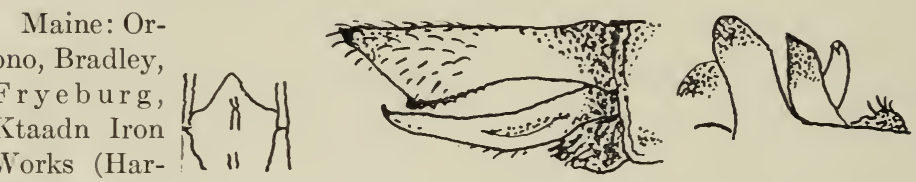

vey); Man-

chester (Wadsworth); Brunswick (Hagen); York Harbor (Moore); Mt. Katahdin (U. S. N. M.).

New Hampshire: Franconia (Slosson); Fabyan's (Calvert); Meredith Neck, Jackson, Little Lake Sunapee (Howe); Intervale (Allen); Kingston (Morse); Manchester (Burnham).

Massachusetts: Milton (Hagen); Fall River, Westport (Holt); West Peabody (Little); Hingham (Barnes); Blue Hills (Clench); Brookline (Galbraith); Woods Hole (Benedict); Sherborn, Wellesley (Morse); Framingham (Warden); Concord, Chatham (Howe); Sharon (Atwater); Rutland (Johnson); Bridgewater (Cushman); Manomet (Brooks); Wareham (Bangs); Cambridge (Atwater); Natick (M. C. Z.); Arlington (Bullard); Provincetown, Nantucket (Calvert).

Rhode Island: Providence (Davis); Wickford (Atwater); Bristol (Howe).

Connecticut: Litchfield (Woodruff); Bethlehem (Benedict); New London (Osburn).

b. Wings over $25 \mathrm{~mm}$. long. 


\section{Sympetrum ambiguum (Ramb.)*}

Ins. Neur. 106. 1842. Type locality: not given.

One record, 一

June 20, 1821.

Massachusetts: Milton (Hagen)

Genus: Leucorrhinia Brittinger.

Me. N. H. Vt. Mass. R. I. Conn.

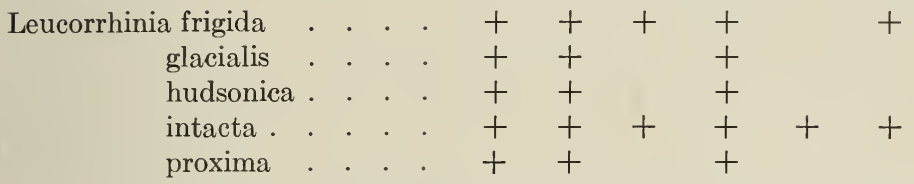

A. Male with yellow triangular spot on 7th. segment of abdomen in adults.

a. $0^{7}$ with abdominal segments $4-6$ black, in both sexes stigma dark brown.

1. Under lip black with sides white.

\section{Leucorrhinia intacta (Hagen).}

Syn. Neur. No. Amer. 179. 1869. Type locality: "Wisconsin; Chicago; Ohio; Massachusetts."

Common, - b r o o k s , ponds, lakes.

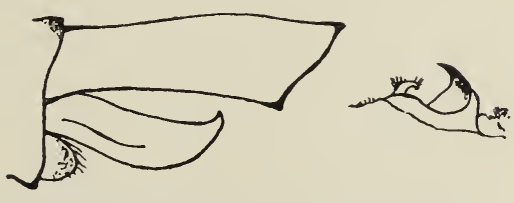

Male abdominal appen- Male genital hamule. dages. Side view. Side view.

May 17 to August 3.

Maine: Orono, Bradley, Westbrook (Harvey); Manchester (Wadsworth); So. West Harbor (Johnson); Bar Harbor (Johnson).

New Hampshire: Mt. Washington (Slosson); Franconia (Slosson, Howe); Manchester (Burnham); Meredith Neck, Moultonboro, Franconia, Lyman, Center Harbor (Howe); Intervale (Allen); Peterboro (Cabot); Meredith (Calvert).

Vermont: Brandon (Dutton).

Massachusetts: (Hagen); Nantucket, Concord, Belmont, Chatham (Howe); Fall River (Holt); Mt. Greylock, Stockbridge (Calvert); Cotuit (Pierson); Woods Hole (Benedict, Osburn); Melrose Highlands (Clemens); Green Lodge, Sherborn, Natick, Winchendon (Morse); Southbridge (Bromley); Framingham (Warden, Frost); North Reading, Dedham (Johnson); Wareham (Bangs); Newtonville (Thaxter); Natick, Tyngsboro, Provincetown, Magnolia (M. C. Z.); Boston, Revere (Scudder); Belmont (Bullard); Wollaston (Sprague).

* Given in recent lists as $S$. albifrons. 
Rhode Island: Bristol (Johnson, Howe); Wickford (Johnson).

Connecticut: Litchfield (Woodruff); Sachem Head (Calvert); Darien (Johnson); New London (Osburn).

b. $\sigma^{7}$ with abdominal segments $4-7$ with wedge shape red spots, in both sexes stigma dull red.

\section{Leucorrhinia hudsonica (Selys).}

Rev. odon. Eur. 53. 1850. Type locality: "Le nouveau Brunswick, pres de la Baie d'Hudson."

Rare,- -bog margined ponds and lakes.

May 30 to July 15.

Maine: Oldtown, Orono (Harvey); Capens (Johnson).

New Hampshire: Mt. Wash-

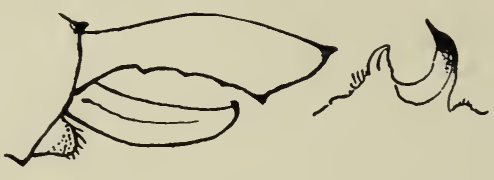
ington, Franconia (Slosson).

Massachusetts: (Hagen); Winchendon (Russell).

B. Male with segments 4-10 black.

a. Under lip entirely black.

1. Abdomen slender, 23-25 mm. long.

\section{Leucorrhinia glacialis Hagen.}

Trans. Amer. ent. soc. 17: 234. 1890. Type locality: "Massachusetts."

Common,- $\mathrm{p}$ o $\mathrm{n} \mathrm{d} \mathrm{s}$, lakes.
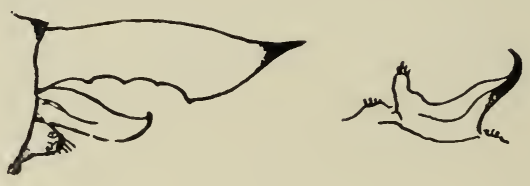

May 19 to August 14.

Maine: Orono, Bradley (Harvey); Echo Lake, Mt. Desert (Johnson); Bar Harbor (Johnson).

New Hampshire: Mt. Washington, Franconia, Crawford Notch (Slosson); White Mts. (Hagen); North Conway (Allen); Lonesome Lake, Lincoln, Moultonboro (Howe); Meredith (Calvert).

Massachusetts: (Hagen); Concord (Howe); Sharon (Atwater).

2. Abdomen spatulate, 20-21 $\mathrm{mm}$. long.

\section{Leucorrhinia frigida Hagen.}

Trans. Amer. ent. soc. $17: 231.1890$. Type locality: "Massachusetts; n. Red River, British America; Ontario; Dakota."

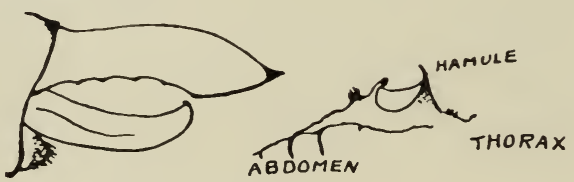


Common,- ponds, lakes.

June 7 to August 9.

Maine: Bradley (Harvey); Mr. Desert (Bullock); Echo Lake, Mt. Desert (Johnson); Bar Harbor (Johnson).

New Hampshire: Mt. Washington; Franconia (Slosson); Moultonboro (Howe); North Conway (Allen); Meredith (Calvert).

Vermont: Brandon (Dutton).

Massachusetts: Sherborn (Babcock); Concord (Howe); Wareham (Bangs); Sharon (Atwater); Hyannisport, Mt. Everett, Guilder's Pond (Johnson).

Connecticut: Litchfield (Woodruff).

b. Under lip black, with sides white.

\section{Leucorrhinia proxima Calvert.}

Trans. Amer. ent. soc. 17: 232.1890. Type locality: "Manchester, Kennebec Co., Maine....Pictou, Nova Scotia."

Rare,- - bog margined ponds and lakes.

June 2 to July 24.

Maine: Oldtown, Orono (Harvey); Manchester (Wadsworth); Bar Harbor (Johnson).

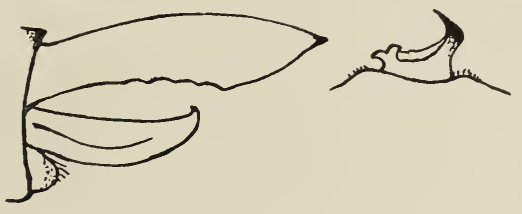

New Hampshire: Mt. Washington, Franconia (Slosson); White Mts. (Hagen).

Massachusetts: Worcester (Hitchings).

Genus: Celithemis Hagen.

Me. N. H. Vt. Mass. R. I. Conn.

Celithemis elisa

eponina

monomelaena . .

ornata

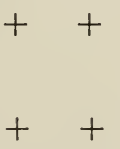

$\begin{array}{lll}+ & + & + \\ + & + & + \\ + & & +\end{array}$

A. Wings spotted with brown from base to tip.

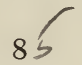


a. Wings more or less suffused with yellow.

1. Wings under $27.5 \mathrm{~mm}$. long.

\section{Celithemis elisa Hagen.}

Syn. Neur. No. Amer. 182. 1861. Type locality: "Bergen Hill, New Jersey; Chicago; New York."

Common,- - brooks, ponds.

May 19 to August 21.

Maine: Bradley (Harvey); Mercer (Davis); Manchester (Wadsworth); Mt. Desert (Bullock); Kennebunkport (?).

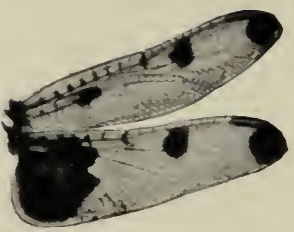

New Hampshire: Derry (Burnham); Moultonboro (Howe).

Massachusetts: Concord (A. N. S., Phila., Howe); Cambridge, Natick (Hagen); Worcester (Hitchings); Woods Hole (Calvert, Osburn); Cuttyhunk (Ufford); Blue Hills (Clench); Tyngsboro (Howe); Provincetown (Morse, Benedict); Dover, Sherborn, West Chop (Morse); Webster (Bromley); Fall River (Holt); South Orleans, Brewster (Howe); Southbridge (Bromley); Woods Hole (Cushman); Hyannisport, Edgartown (Johnson); Framingham (Warden); Sharon (Atwater); Sherborn (Smith); Wareham (Bangs); Natick (M. C. Z.); Wollaston, Milton (Sprague).

Rhode Island: Bristol (Parker, Howe); Buttonwoods (Johnson).

Connecticut; Sachem Head (Calvert); Litchfield, New Hartford (Woodruff); Bethlehem, Burchard's Pond, Fairfield Co. (Benedict); New London (Osburn).

2. Wings over $31 \mathrm{~mm}$. long.

150. Celithemis eponina (Drury).

Ill. nat. hist. 2: 86. 1773. Type locality: "Boston in New England."

Common,-- ponds.

June 21 to September 3.

Massachusetts: Saugus, Boston, Natick (Hagen); Sherborn (Babcock); Worcester (Hitchings); Concord (Howe); Fall River (Holt); Wellesley (Morse);

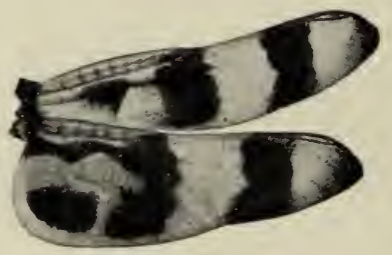

Wareham (Bangs); Auburndale (Johnson); West Peabody (Little); Woods Hole (Osburn); Saugus (M. C. Z.). 
Rhode Island: Block Island (Woodruff).

Connecticut: Litchfield Co., Bethlehem (Benedict); New London (Osburn).
b. Wings not suffused with yellow.

\section{Celithemis monomelaena Willm.}

Ohio nat. 10: 155. 1910. Type locality: Whitley County, Indiana."

Rare,--

July 8 to August 14.

Massachusetts: Wareham (Bangs).

Connecticut: No. Guilford (Howe).

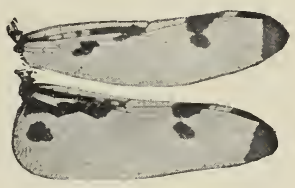

\section{Celithemis ornata (Ramb.).}

Ins. Nevr. 96. 1842. Type locality: "L'Amerique septentrionalis."

Rare,-

July 2 to August 14 .

Maine: Bradley (Harvey); Manchester (Wadsworth).

New Hampshire: Manchester (Burnham).

Massachusetts: Dracut (B. S. N. H.); Sherborn (Morse); Wareham (Bangs); Fall River (Holt); Hyannisport (Johnson); South Orleans (Howe).

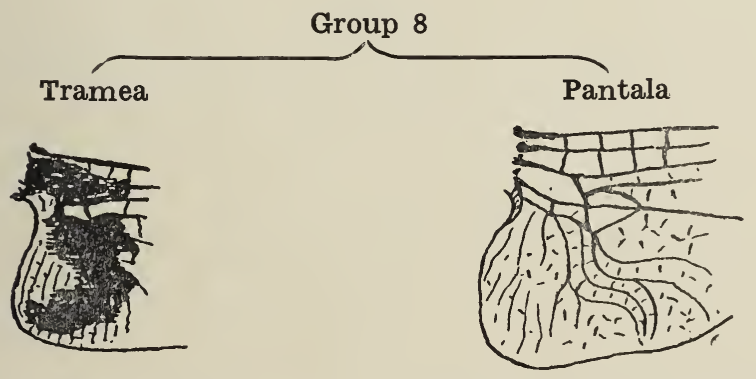

Hind wings dark at base.

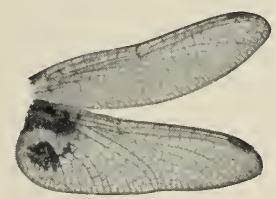


Genus: Tramea Hagen.

Tramea abdominalis . . . .

Me. N. H. Vt. Mass. R. I. Conn. carolina lacerata

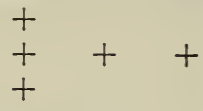

A. Front wings clear. a. Hind wings with dark narrow (6 $\mathrm{mm}$.) basal band.

153. Tramea abdominalis (Rambur).

Ins. Nevr. 37. 1842. Type locality: "des Antilles...L'Amerique septrionalis.... Cuba."

One record.

August 30 .

Massachusetts: Nantucket (Hagen).

b. Hind wings with dark wide $(10 \mathrm{~mm}$.) basal band.

\section{Tramea carolina (Linn.).}

Amoen. acad. 6: 411. 1763. Type locality: "Carolina."

Uncommon, - ponds, migratory a long coast in autumn.

June 1 to September 8 .

Massachusetts: Natick (Hagen); Concord, Chatham (Howe); Wareham (Bangs).

Rhode Island: Matoonoc ? (Simonds); Middletown (Howe).

Connecticut: Sachem Head (Calvert); Darien (Johnson); Burchard's pond, Fairfield Co. (Benedict); New London. (Osburn).

B. Front wings with dark spot at base.

155. Tramea lacerata Hagen.

Syn. Neur. No. Amer. 145. 1861. Type locality: "Pecos River, Western Texas," etc.

One record,-

September 6, 1868 .

Massachusetts: Chelsea (Sanborn, B. S. N. H.). 
Genus: Pantala Hagen.

Me. N. H. Vt. Mass. R. I. Conn.

Pantala flavescens . . . . . $+{ }_{+}+$

156. Pantala flavescens (Fabr.).

Ent. syst. suppl. 285. 1798. Type locality: "India."

Uncommon,- ponds.

August 9 to September

New Hampshire: Amoskeag Falls (Burnham).

Massachusetts: Boston (Cabot); Wareham (Bangs).

Rhode Island: Bristol (teneral) (Howe).

\section{Seasonal Distribution in New England}

ZYGOPTERA

Earliest Species

Agrion maculatum April 16

$\begin{array}{cccccccc} & \text { April } & \text { May } & \text { June } & \text { July } & \text { Aug. } & \text { Sept. } & \text { Oct. } \\ \text { Total number flying in } & 1 & 16 & 30 & 33 & 30 & 15 & 2\end{array}$

\section{ANTSOPTERA}

Earliest Species

Williamsonia lintneri April 30

April May June July Aug. Sept. Oct. Nov.

$\begin{array}{lllllllll}\text { Total number flying in } & 1 & 21 & 52 & 58 & 43 & 20 & 7 & 1\end{array}$

$\begin{array}{lllllllll}\text { Total Odonata flying in } & \overline{2} & \overline{37} & \overline{82} & \overline{91} & \overline{73} & \overline{35} & \overline{9} & \overline{1}\end{array}$ 


\section{Corrections and Additions}

\section{CORRECTIONS}

The Rhode Island and Connecticut records at the top of page 60 belong at the bottom of the page under S. tenebrosa.

\section{ADDITIONS}

\section{PART I}

Agrion aequabile

Massachusetts: Chelmsford (Howe).

Agrion maculatum

Maine: Bar Harbor (Johnson,Merrill).

Massachusetts: Sharon (Atwater); Chatham (Howe); Forest Hills (Henshaw).

Lestes eurinus

June 1 to July 21

Massachusetts: Framingham (Warden).

Lestes unguiculatus

September 8

Massachusetts: Framingham (Warden); Chatham (Howe).

Rhode Island: Middletown, Bristol (Howe).

Lestes uncatus

Maine: Bar Harbor (Johnson).

Vermont: Leiscester (Dutton).

\section{Lestes forcipatus}

\section{PART II}

Massachusetts: Framingham (Warden); Chatham (Howe).

Rhode Island: Howe).

Lester rectangularis

Massachusetts: Framingham (Warden); Chatham (Howe).

Rhode lsland: Warren (Howe).

Lestes vigilax

September 7

Massachusetts: Eastham, South Orleans, Brewster (Howe); Framingham (Warden).

Rhode Island: Bristol (Howe).

Argia moesta

Maine: Mt. Desert (Merrill).

Argia violacea

September 13

Maine: Bar Harbor (Johuson, Merrill).

Massachusetts: Framingham (Wardeu); Orleans (Howe).

Enallagma durum

Massachusetts: South Orleans (Howe).

Enallagma hageni

Maine: Bar Harbor (Johnson).

Massachusetts: Concord (Howe).

Enallagma calverti

Massachusetts: Pepperill (Howe).

Enallagma ebrium

Maine: Mt. Desert (Merrill).

Massachusetts: Framingham (Warden); Belmont (Howe).

Rhode Island: Bristol (Howe).

\section{Enallagma traviatum}

June 19

Enallagma minusculum

Maine: Mt. Desert (Merrill)

Massachusetts: Eastham, South Orleans (Howe). 


\section{Enallagma doubledayi}

August 23

Masachusetts: South Orleans (Howe).

Enallagma civile

Maine: Mt. Desert (Merrill).

Massachusetts: Brookline, Chatham, Orleans, South Orleans (Howe); Sharon

(Atwater); New Bedford (Holcomb); Framingham (Warden); Truro (M. C. Z.).

\section{Enallagma geminatum}

June 15

Framingham (Warden).

Enallagma exsulans

Maine: Mt. Desert (Merrill).

Enallagma signatum

May 26

Massachusetts: Chatham (Howe).

Nehalennia irene

Maine: Bar Harbor (Johnson).

Vermont: Brandon (Dutton).

Massachusetts: Chatham (Howe).

Nehalennia gracilis

Vermont: Brandon (Dutton).

Massachusetts: Cedar Pond, Wenham (Morse).

Amphiagrion saucium

Vermont: Brandon (Dutton).

Chromagrion conditum

May 30

Ischnura verticalis

Maine: Bar Harbor (Johnson); Mt. Desert (Merrill).

Vermont: Leicester (Dutton).

Massachusetts: Brookline, Belmont, Wellfleet, Eastham, Brewster, Chatham

(Howe); Sharon (Atwater); Framingham (Warden); Truro (M. C. Z.).

Rhode Island: Bristol, Warren (Howe).

Ischnura posita

Massachusetts: Chatham, Wellfleet (Howe).

Rhode Island: Warren, Bristol (Howe).

Anomalagrion hastatum

Rhode Island: Middletown, Bristol (Howe).

\section{Cordulegaster maculatus}

\section{Part III}

Massachusetts: Pepperill (Hawker).

Progomphus obscurus

Massachusetts: South Orleans (Howe).

Gomphus parvulus

Vermont: Starksboro (Dutton).

Gomphus exilis

Maine: Bar Harbor (Johnson).

Massachusetts: Sharon (Atwater).

Connecticut: Pomt ret (Howe).

\section{Dromogomphus spinosus}

Vermont: Brandon (Dutton).

Massachusetts: Framingham (Warden); Sharon (Atwater).

\section{Part IV}

In tabular plate of Zygoptera the words Aeshnidae and Libellulidae should be reversed.

Page 30: for femora on diagram read femur.

Basiæschna janata

New Hampshire: Meredith (Calvert).

Massachusetts: Carver (Shurtleff). 


\section{Boyeria vinosa}

September 15

New Hampshire: Jackson (Skinner).

Massachusetts: Brookline (Shurtleff).

Connecticut: Twin Lakes (Calvert).

\section{Gomphaeschna furcillata}

May 4 to July 9

New Hampshire: Meredith (Calvert).

Massachusetts: Sherborn (Smith).

Anax junius

May 4

Maine: Iron Bound Island (Hopkins).

Massachusetts: Chelmsford, Orleans, Chatham (Howe); Framingham (Warden)

Nantucket (Bangs).

Rhode Island: Warren (Howe).

Connecticut: Lakeville (Calvert).

\section{Aeshna umbrosa}

Maine: Mt. Desert (Merrill); Iron Bound Island (Hopkins).

New Hampshire: Jackson (Calvert).

Vermont: Rutland (Johnson).

Massachusetts: Framingham (Warden); Orleans (Howe); Woods Hole (Calvert).

Connecticut: Twin Lakes (Calvert).

\section{Aeshna interrupta}

Maine: Iron Bound Island (Hopkins).

New Hampshire: Jackson (Skinner).

\section{Aeshna clepsydra}

Massachusetts: Chatham (Howe); Framingham (Warden); Brookline (Shurtleff);

Woods Hole (Calvert); Wellfleet (M. C. Z.).

Aeshna eremita

New Hampshire: Jackson (Calvert).

\section{Aeshna verticalis}

Massachusetts: Brookline (Shurtleff); Lexington (Banks).

Strike out one: Manchester (Wadsworth).

\section{Aeshna canadensis}

June 22

New Hampshire: Meredith (Calvert).

Vermont: Leicester (Dutton).

Massachusetts: Chatham (Howe); Woods Hole (Calvert).

\section{Aeshua tuberculifera}

Connecticut: Lakeville (Calvert).

\section{Didymops transversa}

Maine: Bar Harbor (Johnson).

Massachusetts: Chelmsford (Howe).

Dorocordulia lepida

New Hampshire: Meredith (Calvert).

Dorocordulia libera

June 7 to August 29

Maine: Bar Harbor (Johnson).

New Hampshire: Meredith (Calvert).

Vermont: Brandon (Dutton).

Massachusetts: Wareham (Bangs); Fall River (Holt); Lexington (Banks).

\section{Epiaeschna heros}

Massachusetts: Lexington (Banks).

\section{Epicordulia princeps}

Massachusetts: Chatham (Howe).

Somatochlora forcipata

Vermont: Brandon (Dutton). 


\section{Somatochlora kennedyi}

May 30

New Hampshire: Meredith (Calver॰).

\section{Cordulia shurtleffi}

Maine: Bar Harbor (Johnson).

New Hampshire: Meredith (Calvert).

\section{Tetragoneuria cyanosura}

Vermont: Brandon (Dutton).

Massachusetts: Orleans (Howe): Milton (Wardeu)

Connecticut: Pomfret, Webster (Howe).

\section{Tetragoneuria cyanosura simulans}

New Hampshire: Meredith (Calvert).

\section{Tetragoneuria spinigera}

July 23

New Hampshire: Meredith (Calvert).

Page 54 before line No. 100 Dorocordulia lepida (Hagen), read

A. Last abdominal segments not spatulate. before line No. 101 Dorocordulia libera (Selys), read

B. Last abdominal segments spatulale.

\section{Recapitulation of New England Libellulidae (Libellulinae), and Odonata.}

Maine

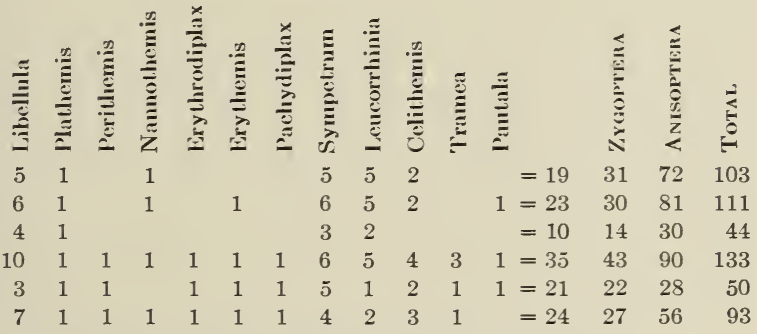

Total Odonate Fauna for New England $=156$ species.

\section{Bibliography of New England Faunal Papers.}

1866: Scudder, S. H. Notes on some Odonata from the White Mountains of New Hampshire, Proc. Bost. Soc. Nat. Hist. 10: 211-222.

1867: Scudder, S. H. Additional Remarks upon the Odonata of the .... White Mountains of New Hampshire, Proc. Bost. Soc. Nat. Hist., 11: 298-300.

1873: Hagen, H. A. Report on Mr. H. S. Scudder's Odonata from the White Mountains, af ter an examination of the Types, Proc. Bost. Soc. Nat. Hist. 15 : 376-377.

1890: Wadsworth, M. List of the Dragonflies (Odonata) taken at Manchester, Kennebeck Co., in 1888 and 1889, Ent. News 1. 36-37, 55-57.

1891: Harvey, F.LeR. Contributions to the Odonata of Maine, I, Ent. News 2: $50-51$

1891: Harvey, F. LeR. Contributions to the Odonata of Maine, I, ibid, 2 : 73-75.

1892: Harvey, F. LeR. Contributions to the Odonata of Maine, II, ibid, 3: 91-93.

1892 - Harvey, F. LeR. Contributions to the Odonata of Maine, II, ibid, 3: 116-117. 
1898: Harvey, F. LeR. Contributions to the Odonata of Maine, III, ibid, 9: 59-64.

1900: Burnham, E. J. Preliminary Catalogue of the Anisoptera of Manchester, N. H. Manchester Inst. Arts \& Sci. 1: 27-38.

1901: Burnham, E. J. Additional Notes on the Anisoptera in the Vicinity of Manchester, N. H. Proc. Manchester Inst. Arts \& Sci. 2 : 37-45.

1901: Harvey, F. LeR. Contributions to the Odonata of Maine, IV, Ent. News 12: 178-179.

1901: Harvey, F. LeR. Contributions to the Odonata of Maine, IV, ibid, 196-198.

1901: Harvey, F. LeR. Contributions to the Odonata of Maine, IV, ibid, 239-243.

1902: Harvey, F. LeR. A Catalogue and Bibliography of the Odonata of Maine with an Annotated List of their Collectors, University of Maine Studies, No. 4.

1905: Calvert, P. P. Fauna of New England 6. List of Odonata. Occasional Papers, Bost. Soc. Nat. Hist. 7: 1-43.

1914: Woodruff, L. B. Some Dragonflies of a Connecticut Brook, Journ. N. Y. Ent. Soc. 22 : 154-159.

1915: Pierson, E. L. Jr. A List of Odonata (Dragon Flies) collected at Concord, Mass. Proc. Thoreau Mus. Nat. Hist. 1: 41.

1916: Howe, R. H. Jr. A Preliminary List of the Odonata of Concord, Mass. Psyche 23: 12-15.

1917: Howe, R. H. Jr. Distributional Notes on New England Odonata, Part I. Psyche 24: 45-53.

1917: Howe, R. H. Jr. Manual of the Odonata of New England, Part I, Memoir Thoreau Mus. Nat. Hist. II, 1-8.

1917: Howe, R. H. Jr. Manual of the Odonata of New England, Part II, ibid, 9-24.

1918: Howe, R. H. Jr. Manual of the Odonata of New England, Part III, ibid, 25-40.

1918: Howe, R. H. Jr. The Dragon and Damsel-flies (Odonata) of Nantucket, Mass. Annual Report Maria Mitchell Assoc. of Nantucket, unpaged, May.

1918: Howe, R. H. Jr. Distributional Notes on the New England Odonata, Part II, Psyche 25: 106-110.

1919: Howe, R. H. Jr. The Odonata of Concord, Massachusetts, Ent. News, 30: 10-14.

1919: Howe, R. H. Jr. Odonata of the Franconia Region, New Hampshire, Can. Ent. 51: 9-15.

1919: Howe, R. H. Jr. Addition to the Odonata of Franconia Region, N. H., ibid, 50: 93 .

1919: Howe, R. H. Jr. A List of Dragon-flies collected at Wareham, Mass., during the Years 1911 to 1913 by Mr. Outram Bangs. Psyche 26: 65-68.

1919: Howe, R. H. Jr. Damsel and Dragon Flies, List of Specimens Recorded in this Town, [Bristol, R. I.] The Bristol Phoenix, August 19.

1919: Howe, R. H. Jr. Manual of the Odonata of New England, Part IV, ibid, 41-66.

(To be continued) 


\section{Memoir of the Thorean Musem of Natural History: II}

Manual of the Odonata of New England

By R. HEBER HOWE, Jr.

\section{PART IT}

MARCH 15,1920 .

\section{LARVAE OR NYMPHS}

In this Manual have been treated only the adult or imago damsel and dragonflies. The nymphs which have had far less study are figured here only to show the Order characteristics. At some later date a complete treatment of them may be undertaken. The larvae or nymphs of the Odonata are aquatic. They inhabit ponds, lakes, brooks, and rivers, and their exuviae after the transformation of the imagos, are left attached to the stem of water plants, dead wood, or rocks, and are familiar to every student of nature. The works of Dr. J. A. Needham, Mr. Louis Cabot, and Dr. C. H. Kennedy include studies of many species, and will prove of much help in the study of the nymphs. The latter has kindly allowed me to reproduce drawings made by him.

\section{Order: Zygoptera}

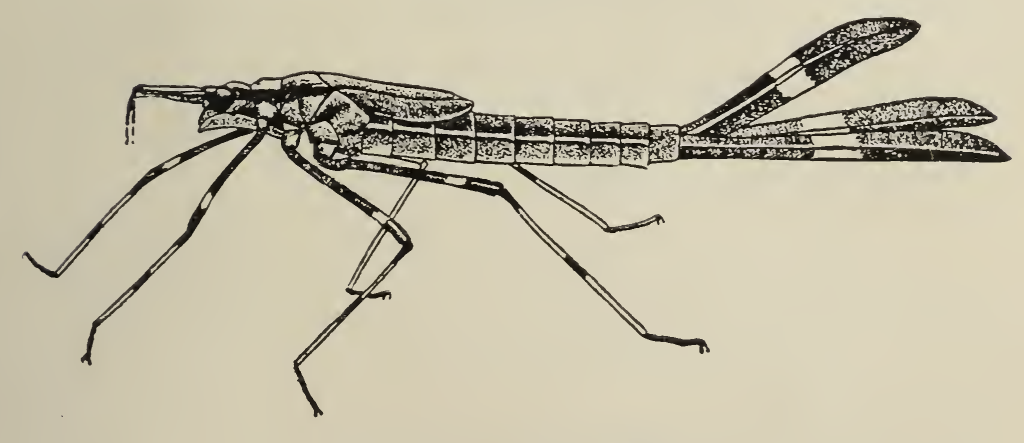

A nymph of the Genus: Agrion 
()rder: Anisoptera

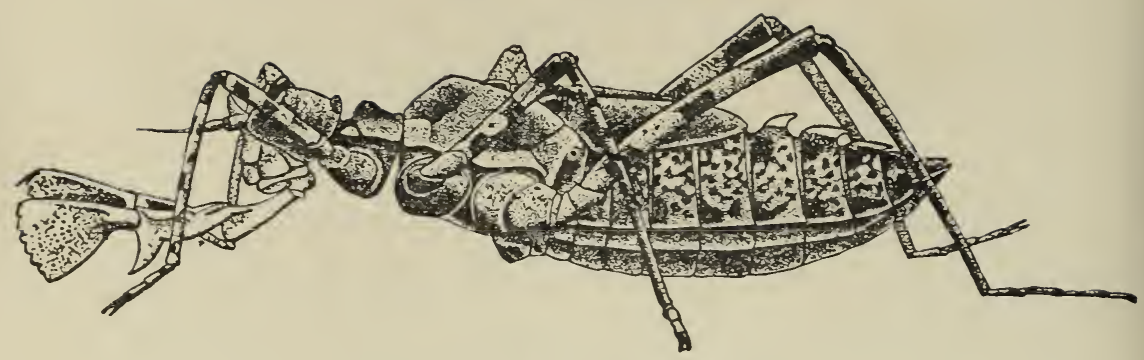

A nymph of the Genus: Macromia

\section{Additions and Corrections}

Agrion aequabile

\section{l'IRT I}

Massachusetts: Winchendon (Russell).

Agrion maculatum

Vew IIampshire: Keene (Wheelock).

Massachusetls: Winchendon (Russell).

Lestes eurinus

IIassacluselts: Winchendon (Russel])

Lestes forcipatus

Maine: Bar Harbor (Jolınson).

Massachusetts: West Peabody (Litlle); Winchendon (kussell).

Lestes congener

Massachusetts: Winchendon (Russell).

Lestes uncatus

Massachusetts: Winchendon (Russell)

PART II

Lestes vigilax

Massachusetts: West Peabody (Little).

Argia violacea

Massichusetts: Winchendon (Russell).

Page 21: Unter Ischnura for " 's with more than 7 postcubitals " read with more than $s$ postcubitals in hind wings.

Under Acomalagrion for " 's $^{\circ}$ with less than 7 postcubitals " read with 8 or less postcubitals in hind wings.

Ischnura verticalis

Massachusetts: Winchendon (Russell).

Rhode Island: Wickford (Atwater).

Ischnura posita

Rlode Island: Wickford (Atwater).

Cordulegaster diastatops

$$
\text { Рмит } 11
$$

Massachusetts: Winchendon (Kussell).

To habitat of Progomphus obscurus add: ponds.

Dromogomphus spinosus

June 16

Page 38 under Enallagma hageni

Massachusetts: strike ont "Franconia (Howe)." 



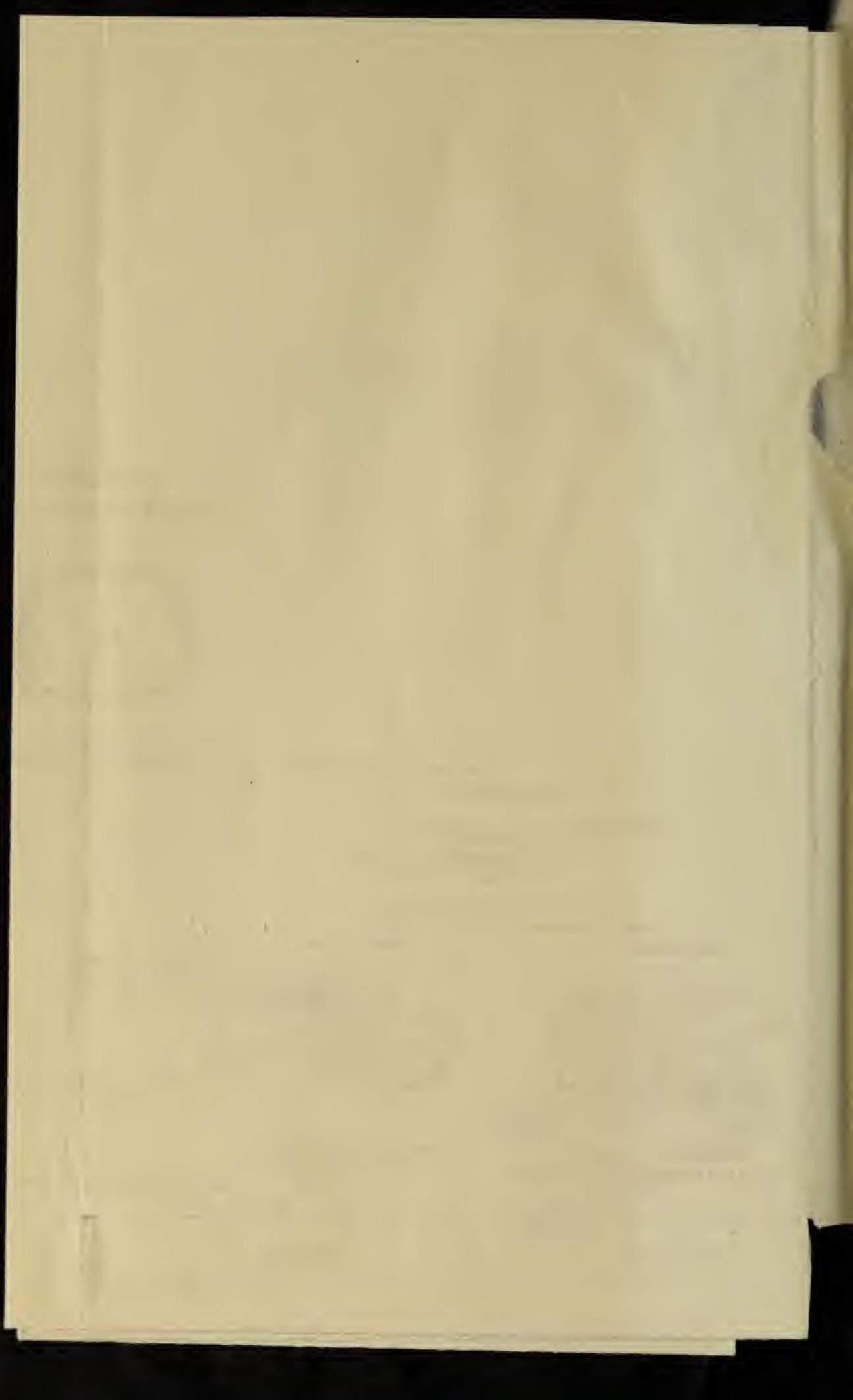


Boyeria vinosa

Massachusetts: Wincheudon (Rnssell).

Anax loagipes

August 27.

Aeshna umbrosa

New Hampslire: Keene (Wheelock).

Massachusetts: Chatham (Howe).

Aeshna canadensis

Massachusetts: Winchendon (Russell).

Rhode Island: Bristol (Howe).

Aeshna constricta

New Hampshire: Fitzwilliam (Barnes)

Massachusetts: Winchendon (Russell).

Dorocordulia lepida

Mainc: Bar Harbor (Jolınson).

Dorocordulia libera

For "August 29" read August 9

Libellula cyanea

Pant V

Massachusetts: Winchendon (Russell).

Libellula exusta

May 28 to July 25 .

Massachusetts: Winchendon (Russell).

Libellula exusta julia

June 4 to July 7

New Hampshire: Keene (Wheelock).

Massachusetts: Winchendon (Russell).

Libellula quadrimaculata

Vermont: Bristol (Duttou).

Massachusetts: Winchendon (Russell).

Libellula pulchella

New Hampshirc: Keene (Wheelock).

Massachusetts: Winchendon (Russell).

Plathemis lydia

New Hampshire: Keene (Wheelock).

Massachusetts: Wincliendon (Russell).

Sympetrum rubicundulum

Massachusetts: Winchendon (Russell).

Sympetrum vicinum

July S

Massichusetts: Winchendou (Mussell).

Sympetrum semicinctum

Massachusetts: Winchendon (Russell).

Leucorrhinia intacta

Massachusetts: Wakefield (Atwater).

Leucorrhinia frigida

Massachusetts: Winchendon (Russell).

Page 87 Group 8 should have been printed in yellow.

Page 91 under Cordulegaster maculata for " (Hawker) " read (Parker)

\section{Bibliography}

1891: Bullock, J. D. Notes on Mt. Desert Dragon-flies, Ent. News 2:93-94.

1891: Wadsworth, M. Additions and Corrections, to the list of Dragon-flies

(Odonata), of Manchester, Kennebec Co., Mainc, Ent. News 2:11-12.

1892: Wadsworth, M. Second Additions and Corrections, etc. Ent. News $3: 8-4$.

1894: Wadsworth, M. Third Addition to the list, ctc. 5:132.

1894: Calvert, P.P. Data on the Distribution of Dragon-flies (Odonati1), Ent.

News 5:I. 242-244.

1898: Wadsworth, M. Fourlh Arldition to the list, els. Ent. News 9:111. 
From the following persons, in addition to those thanked in the prefatory remarks in Part I, I have received much valuable assistance in the preparation of this Manual, and to whom I desire to express my most sincere thanks: Drs. C. H. Kennedy, J. G. Needham, R. C. Osburn, Messrs. S. W. Bromley, C. W. Atwater, D. Merrill, W. G. Low, 3rd., W. J. Clench, F. C. Whitehouse, E. J. Smith, O. Bangs, H. C. Holcomb, and Miss Elizabeth B. Warden. 


\section{INDEX}

(Numbers in blackfaced type refer to the full treatment of the species, those in lighter face to additional dates, stations, or corrections.)

Aeshna caerulea septentrionalis . . . . . . . 47

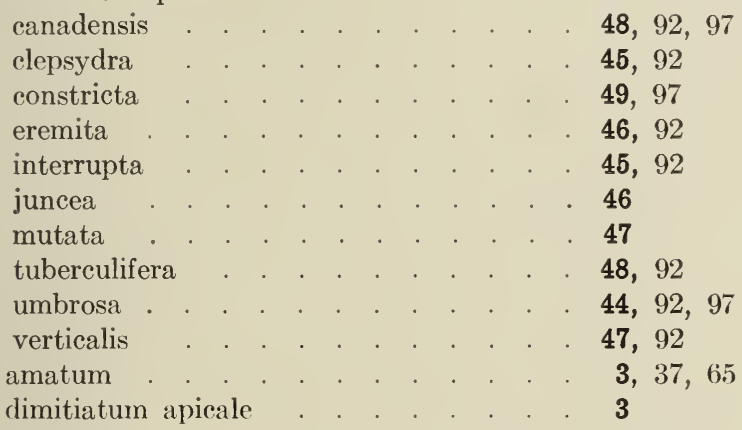

aequabile . . . . . . . . . . $3,37,65,90,96$

maculatum . . . . . . . . . . $4,37,65,90,96$

Amphiagrion saucium : . . . . . . . . 20, $39,66,91$

Anomalagrion hastatum . . . . . . . 22, 40, 66, 91

Anax junius . . . . . . . . . . . . 43, 92

longipes . . . . . . . . . . . . . 44

Argia apicalis . . . . . . . . . . . . . . 13

moesta . . . . . . . . . . . $12,38,65,90$

sedula . . . . . . . . . . 13

violacea . . . . . . . . . . $12,38,65,90,96$

Basiaeschna janata . . . . . . . . . . 42, 91

Boyeria grafiana . . . . . . . . . . . . 42

vinosa . . . . . . . . . 4 $41,92,97$

Celithemis elisa . . . . . . . . . . . 86

eponina . . . . . . . . . . 86

monomelaena . . . . . . . . . 87

ornata . . . . . . . . . . 87

Chromagrion conditum . . . . . . . . 20, $40,66,91$

Cordulegaster diastatops . . . . . . . . . 25, 66, 96

maculatus . . . . . . . . 25, 91,97

obliquus . . . . . . . 26

Cordulia shurtleffi

61,93

Didymops transversa . . . . . . . . . . 51, 92

Dorocordulia lepida . . . . . . . . . . . $54,92,97$

libera . . . . . . . . . 54, 52, 97

Dromogomphus spinosus . . . . . . . . . 36, 91,96 
Enallagma aspersum

calverti

carunculatum

civile

cyathigerum

divagans

doubledayi

durum

ebrium

exulans

geminatum

hageni

laterale

minusculum

pictum

pollutum

signatum

traviatum

Epiaeschna heros

Epicordulia princeps

Erythemis simplicicollis

Erythrodiplax berenice

Gomphus abbreviatus

adelphus

albistylus

hrevis

exilis

fraternu.

furcifer

lividus

notatus

pallidus

parvulus

quadricolor

scudderi

sordidus

spicatus

spiniceps

vastus

ventricosus

Gomphaeschna furcillata

Gomphoides

Hagenius brevistylus

Helocordulia uhler

Hetaerina americana

Ischnura kellicotti
$16,38,65$

$15,38,65,90$

16,39

17, $39,65,91$

14, 38

17, 39

16, 39, 91

$14,38,65,90$

$15,38,90$

18, 39, 91

$17,39,65,91$

$15,38,90,96$

18

$16,39,90$

17, 65

$18,39,65$

$18,39,65,91$

$16,38,65,90$

50, 92

55, 92

77

77

31,66

33 ,

31,66

32,66

32,91

33

34,66

32

35

35

31, 91

32

35

32

34

35, 66

33

33

43,92

27

28, 66

56

5, 37

22 
Ischnura posita

$22,40,66,91,93$

ramburii

22, 40

verticalis

$21,40,66,91,96$

Lestes congener

7, 37, 65, 96

eurinus

$7,37,65,90,98$

disjunctus

$9,37,65$

forcipatus .

9, 37, 65, 90, 93

inequalis

11, 38,65

rectangularis

9, 37, 90

uncatus

8, 37, 65, 90, 96

unguiculatus

8, 11, 37, 65, 90

vigilax

$10,38,65,90,96$

Leucorrhinia frigida

84,97

glacialis

84

hudsonica . . . . . . . 84

intacta

83,97

proxima

85

Libellula auripennis

69

basalis

71

cyanea

70, 97

exusta

71,97

exusta julia

71,97

flavida

70

incesta

69

luctuosa

71

pulchella

73,97

quadrimaculata

72,97

semifasciata

73

vibrans

70

52

Macromia illinoiensis

75

Nasiaeschna pentacantha . . . . . 50

Nehalennia gracilis

20, 91

irene

$19,39,66,91$

Neurocordulia obsoleta

53

yamaskanensis . . . . 53

Ophiogompus anomalus . . . . . . . . . 28

aspersus

30,66

carolus . . . . . . . . . 29

colubrinus . . . . . . . 29

johannus . . . . . . . 29

mainensis . . . . . . . 28

rupinsulensis . . . . . . 29, 66

Pachydipax longipennis . . . . . . . . 78

Pantala flavescens . . . . . . . . . . . . . 89

Perithemis domitia tenera . . . . . . . . 75 
Plathemis lydia

Progomphus obscurus

Somatochlora albicincta

cingulata

elongata

forcipata

franklini

kennedyi

linearis

minor

tenebrosa

walshii

williamsoni

Sympetrum albifrons

ambiguum

costiferum

obtrusum

rubicundulum

scoticum

semicinctum

vicinum

Tachyopteryx thoreyi

Tetragoneuria canis

costalis

cynosura

cynosura semiquea

cynosura simulans

morio

spinigera

spinosa

Tramea abdominalis

carolina

lacerata

Williamsonia lintneri
74, 97

27, 91,96

57

57

58

59, 92

60

59, 93

58

59

60

60

58

83

83

81

80

79,97

80

81,97

82,97

24

64

64

62, 93

63

62,93

63

64, 93

64

88

88

88

53 


\title{
Memoir of the Thoreau Museum of Natural History: II. Nanual of the Odonata of New England ${ }^{1}$
}

By R. Heber Howe, Jr.

\section{NYMPHS}

\author{
ZYGOPTERA
}

\section{PART I}

December 24, 1921

The pictorial keys of the imagos of the Odonata of New England have met with such general and gratifying approval that the author begins with this paper a similar work on the larvae or immature stages of the species treated in the former work. Many of those persons thanked before are giving substantial aid in this portion of the manual, among them particularly to be mentioned are Drs. P. P. Calvert, P. Garman, E. M. Walker, J. G. Needham, C. H. Kennedy, C. B. Wilson.

I $r_{\llcorner}$Part VI of the former manual, page 95, an introductory statement was made in regard to the habitats of the nymphs. In Dr. Garman's valuable work on The Zygoptera, or Damselflies, of Illinois (Bull. Ill. State Lab. Nat. Hist. 12: 411588. Pls. 58-73. 1917), complete descriptions and measurements are given for all but a few species here listed, and for permission to reproduce many of his figures of gill plates the author has both Dr. Garman and Dr. S. A. Forbes to thank. For permission to use the figure of the gill plates of Enrllagma ebrium (Ent. News 26: Pl. 1. 1915) acknowledgement is due to Drs. Calvert and Cresson of the Entomological News. For the use of the figures of egg and first nymphal stage of Enallagma signatum the author is indebted to Dr. C. B. Wilson though the courtesy of the Bureau of Fisheries. For several figures of Lestes, etc., I am indebted to Dr. E. M. Walker. Two of the cuts used in the key are after published drawings by Dr. Kennedy. The full page plates were drawn for the author by Mr. E. N. Fischer.

1 Contribution from the Entomological Laboratory of the Bussey Institution, Harvard University. - No. 192. 
The eggs of the Zygoptera are ovoidal, about $1 \mathrm{~mm}$. long, 200-300 of which are probably laid by one female. They are inserted in a more or less definite arrangement in the leaves of grasses, sedges (bur reed), flag (Iridaceae) above or below (female submerging) the water surface, and require a period of over 14 or 18 days to hatch, but the exact length of incubation is not known, though believed to be from 4-5 weeks.

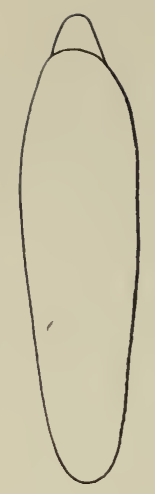

\section{Larvae, Nymphs or Natads}

After hatching the larvae are known as pronymphs.

The nymphal life has been estimated at from 229-624 days. From 10-15 molts are required for full development. Emergence generally takes place before 8 A.M., or after 6 P.M., though the nymphs of this order frequently emerge between these hours. The cast skins found clinging to the stems of aquatic plants are called exuviae.

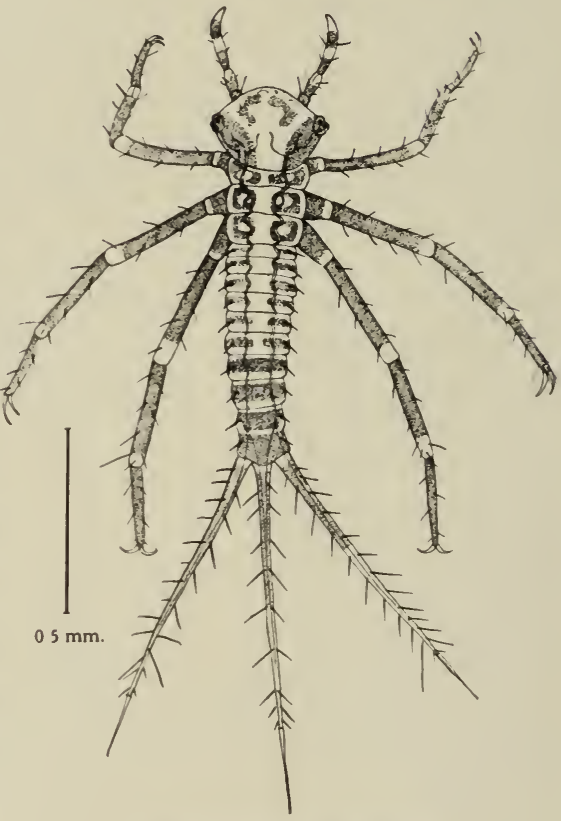

First true nymphal stage 


\section{Food}

The nymphs are both phytophagous and predacious, and have been recorded as feeding upon the following organisms:

$\begin{array}{ccc}\text { PlaNTs } & \text { Copepoda } & \text { Diptera } \\ \text { Algae } & \text { Cyclops } & \text { Nematocera } \\ \text { Diatoms } & \text { Cypris } & \text { Chironomids } \\ \text { Navicula } & \text { Diaptomus } & \text { Ceratopogon } \\ \text { Encyonema } & \text { Phyllopoda } & \text { Orthocladius } \\ \text { Gomphonema } & \text { Cladocera } & \text { Corynoneura } \\ \text { Synedra ulna } & \text { Anomopoda } & \text { Culicids } \\ \text { Fragilaria } & \text { Daphnia } & \text { Culex } \\ \text { Nitzschia acicularis } & \text { Bosmina } & \text { Simuliids } \\ \text { Chlorophyceae } & \text { Pleuroxus } & \text { Brachycera } \\ \text { Desmids } & \text { Simocephalus } & \text { Dolichopdids } \\ \text { Closterium } & \text { Arachnida } & \text { Anthomyids } \\ \text { Oedogonium } & \text { Hydrachinida } & \text { Syrphids } \\ \text { Ulothrix } & \text { Dolomedes } & \text { Geomyzidids } \\ \text { Cyanophyceae } & \text { Hexapoda } & \text { Coleoptera } \\ \text { Oscillatoria } & \text { Ephemerida } & \text { Berosus } \\ & \text { Callibaetis } & \text { Lepidoptera } \\ \text { ANIMALs } & \text { Hexagenia } & \text { Ancyloxcypha } \\ \text { Protozoa } & \text { Odonata } & \text { Mollusca } \\ \text { Paramecium } & \text { Zygoptera } & \text { Physa } \\ \text { Crustacea } & \text { Ischnura } & \text { Planorbis } \\ & \text { Enallagma } & \\ & \text { Hemiptera } & \\ & \text { Aphids } & \end{array}$

\section{ENemies}

The eggs, and later during nymphal life the larvae are the prey of fishes of whose food they often comprise from 10-25 per cent. They are also preyed upon by Protozoa (Vorticella), Coelenterata (Hydra), Vermes (Nematodes), and by certain Hymenoptera, Coleoptera (Dytiscus, Zaitha), aquatic Hemiptera, (Notonecta, Ranatra, Belostoma), Arachnida (including Mites), frogs, newts, reptiles (Chrysemys), and diving birds (Colymbus), as well as by larvae of their own or other Odonate species. They are attacked also by Algae (Oedogonium, Diatoms), and Fungus (Saprolegniales) growths.

\section{Teneral Life}

The freshly emerged imago, called a teneral, has been observed to take five and one half hours to complete its full development. 


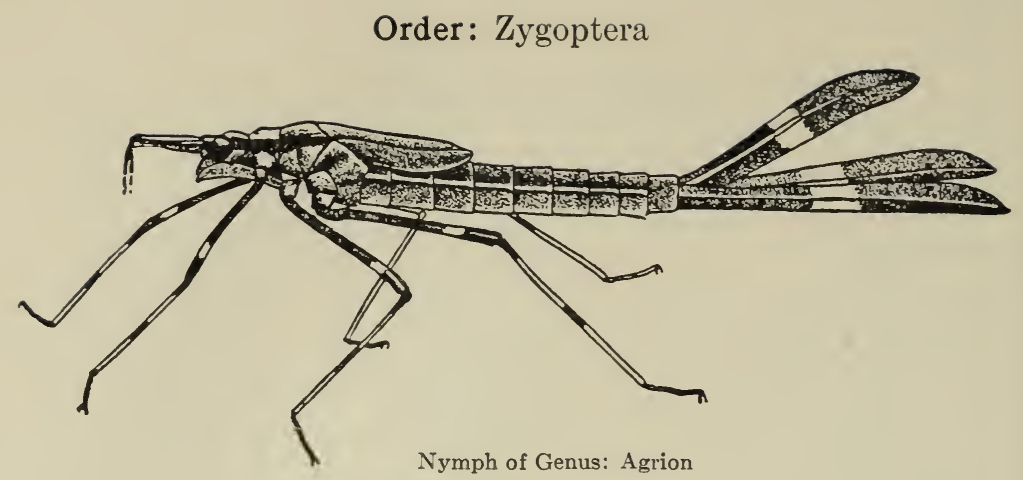

Family: Agrionidae

Subfamily: Agrioninae

Genus: Agrion

Nymphs inhabit running water, - brooks and small streams.

1. Agrion amatum. Nymph unknown.

2. Agrion dimidiatum apicale. Nymph unknown.

$\mathrm{A},-$ Basal segment of antennae longer than head.

3. Agrion aequabile.

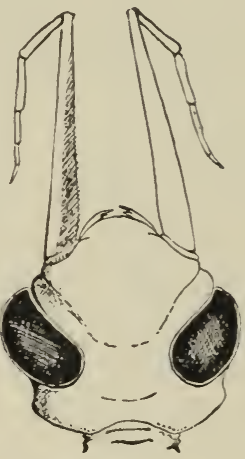

B, - Basal segment of antennae not longer than the head.

4. Agrion maculatum.

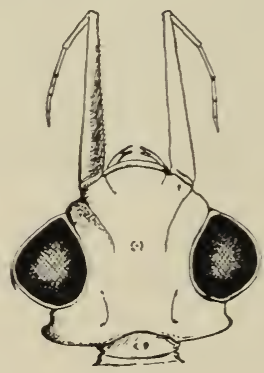




\section{Genus: Hetaerina}

Nymphs inhabit running water, - streams and rivers.

5. Hetaerina americana.

\section{Genus: Lestes}

(For detailed figures see Walker, Can. Ent. 46: pl. 13, 14, 23, 1914.) Nymphs inhabit still water, - ponds and lakes.

A, - Lateral spines never present on abdominal segment two.

Mentum reaching beyond base of coxae.

a. Four or five setae on each lateral labial lobe.

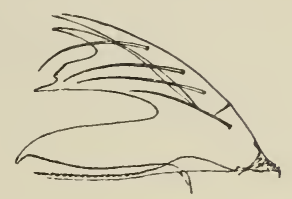

6. Lestes congener.

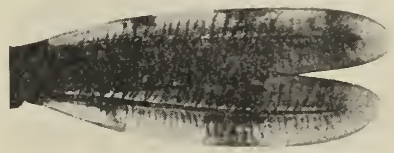

Branchial gill plates

b. Three setae on each lateral labial lobe.

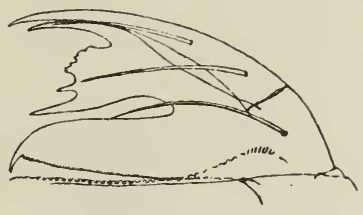

I. Lateral spines always present on abdominal segment four.

\section{Lestes eurinus.}

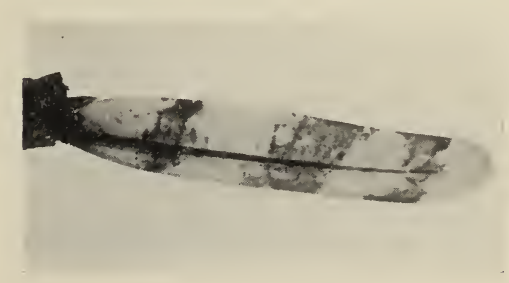


II. Lateral spines never present on abdominal segment four.

1. Mentum of labium reaching to apex of hind coxae, or beyond.

*Mentum of labium reaching to apex of hind coxae

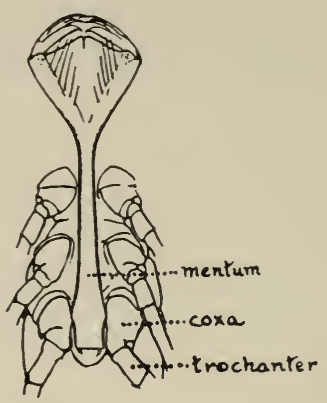

8. Lestes uncatus.

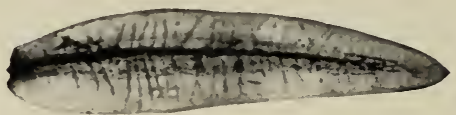

**Mentum of labium reaching beyond apex of hind coxae to apex of trochanter.

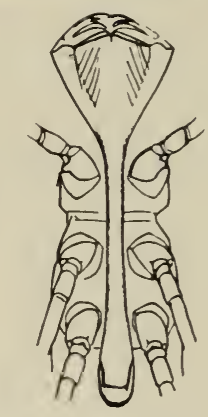

\section{Lestes disjunctus.}

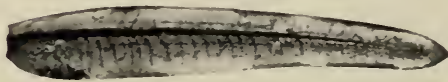

2. Mentum of labium reaching only to middle of hind coxae.

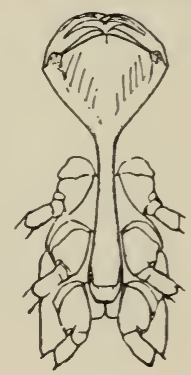


*Mental setae five on each side of labium.

10. Lestes rectangularis.

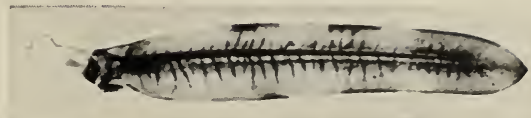

**Mental setae six or seven on each side of labium.

†Gill plates unbanded.

11. Lestes unguiculatus.

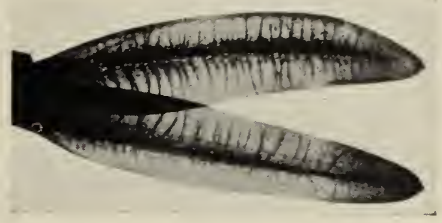

††Gill plates banded.

\section{Lestes forcipatus.}

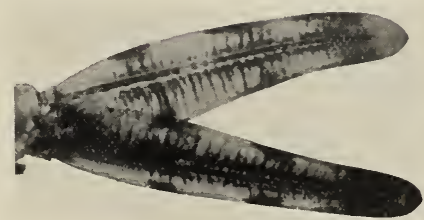

B, - Lateral spines always present on abdominal segment two. Mentum not reaching base of hind coxae.

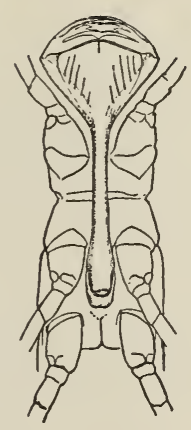

13. Lestes vigilax.

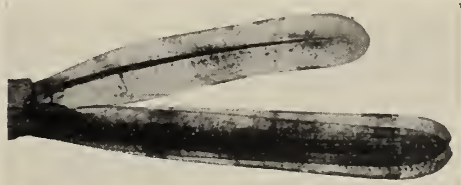

14. Lestes inequalis. Nymph unknown. 
Genus: Argia

Nymphs inhabit ponds and lakes, and slow running and even rapid streams.

A, - Gill plates ovate, tips inconspicuous.

15. Argia moesta.

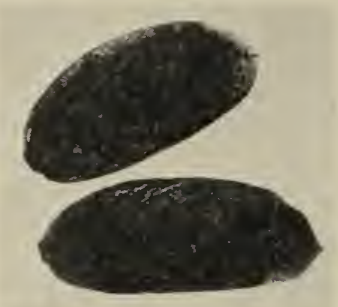

B, - Gill plates spatulate, tips conspicuous.

16. Argia violacea.

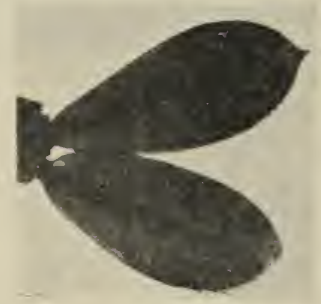

17. Argia translata. Nymph unknown.

Genus: Enallagma

Nymphs inhabit ponds, lakes and streams.

(For key to ten of the following species see Garman, Bull. Ill. State Lab. 12: $517-518,1917$.) No good characters are yet known for the separation of all the species of this genus. The gill plates will serve for identification in most cases.

18. Enallagma aspersum. Nymph unknown.

19. Enallagma calverti.

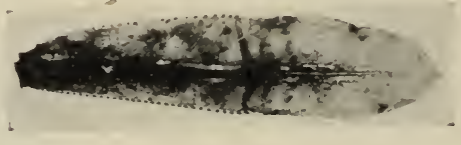


20. Enallagma carunculatum.

21. Enallagma civile.
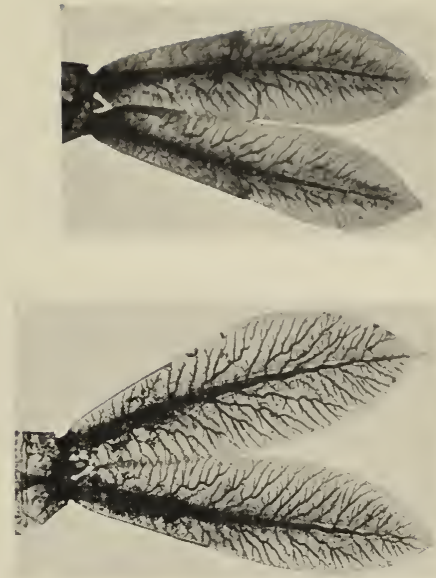

22. Enallagma cyathigerum.

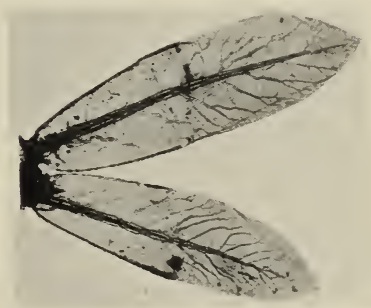

23. Enallagma doubledayi.

24. Enallagma durum.

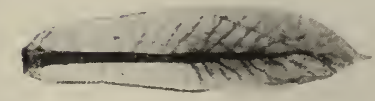

25. Enallagma ebrium.

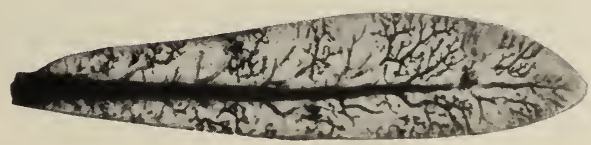


26. Enallagma exsulans.

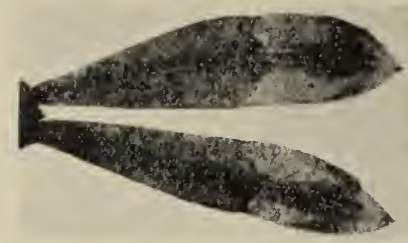

27. Enallagma geminatum.

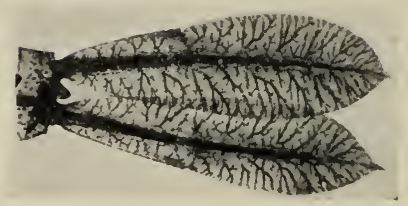

28. Enallagma hageni.

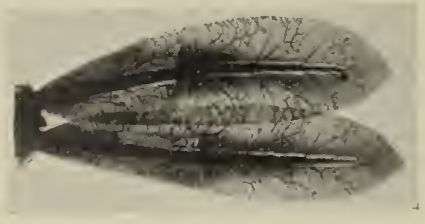

29. Enallagma laterale.

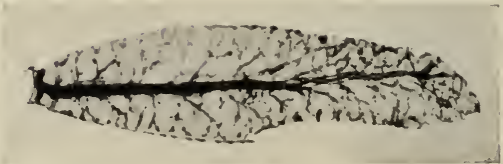

30. Enallagma signatum.

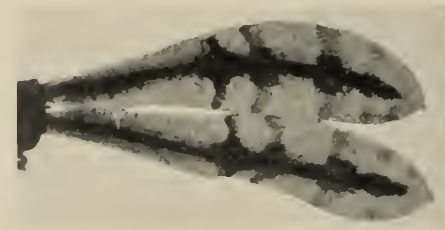

31. Enallagma traviatum

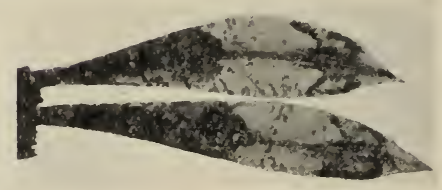


32. Enallagma vesperum

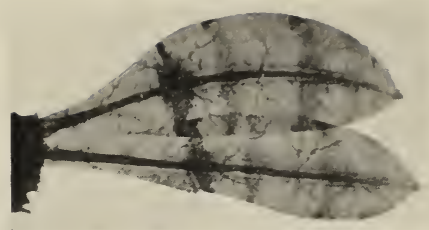

33. Enallagma divagans. Nymph unknown.

34. Enallagma minusculum. Nymph unknown.

35. Enallagma pictum. Nymph unknown.

\section{Genus: Nehalennia}

(For detailed figures see Walker, Can. Ent. 45: pl. 1, 1913.)

Nymphs inhabit spring runs, bogs, grassy ponds and lakes.

A, - Gills unspotted, head with 4 to 6 hind marginal spines.

36. Nehalennia gracilis.

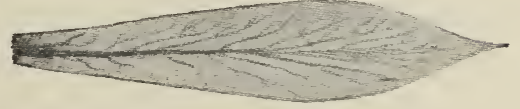

B, - Gills spotted, head with 12 or more hind marginal spines.

37. Nehalennia irene.

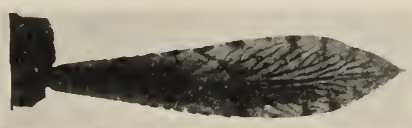

\section{Genus: Amphiagrion}

Nymphs inhabit meadow brooks and marshy bogs.

38. Amphiagrion saucium.

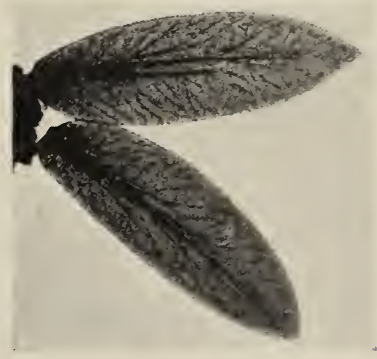




\section{Genus: Chromagrion}

Nymphs inhabit ponds, small lakes and streams.

\section{Chromagrion conditum.}

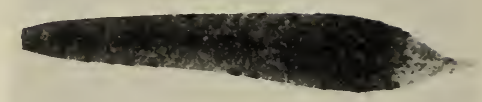

Genus: Ischnura

Nymphs inhabit streams and rivers, ponds and lakes, i.e. permanent water.

A, - Gill plates with strong arcuate bands.

40. Ischnura posita.

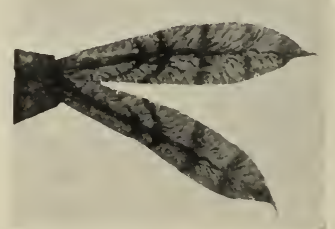

B, - Gill plates with no or only slight arcuate bands.

41. Ischnura verticalis.

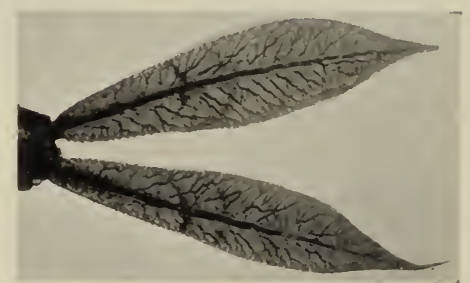

42. Ischnura kellicotti. Nymph unknown.

43. Ischnura ramburii. Nymph unknown.

Genus: Anomalagrion

Nymphs inhabit springy bogs, and stagnant marsh water.

44. Anomalagrion hastatum.

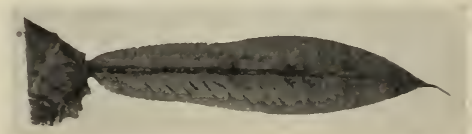


PICTORIAL KEY TO ZYGOPTERA NYMPHS

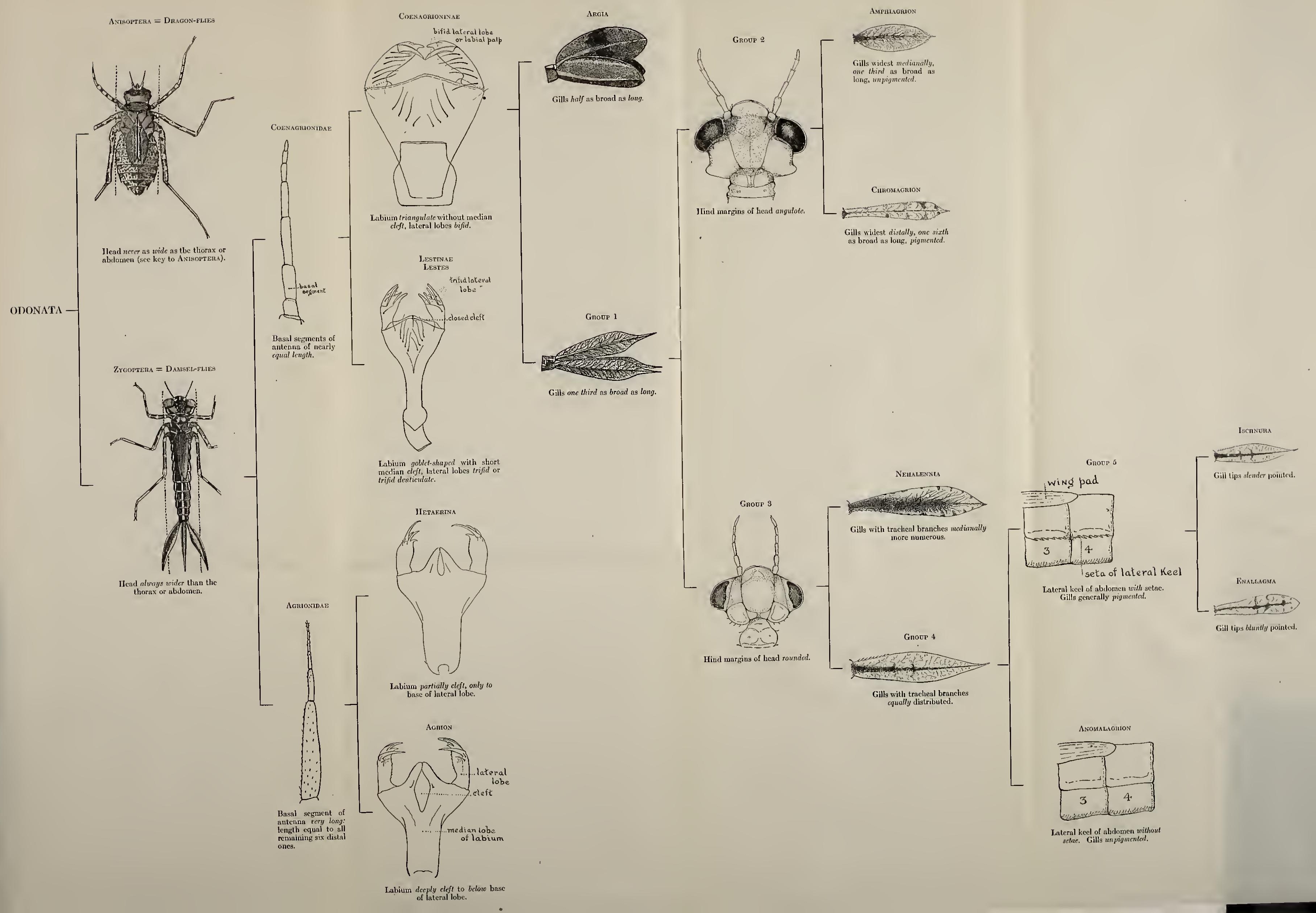



In the following full page illustrations the last few segments of the abdomen are shown rotated, exposing the branchial plates in profile. To the left of each complete drawing of the nymph the closed labium as seen from the ventral side is shown. In plate $\mathrm{X}$, in the complete drawing, the labium is shown extended, and in the drawing of the labium the raptorial setae show through the mentum. 



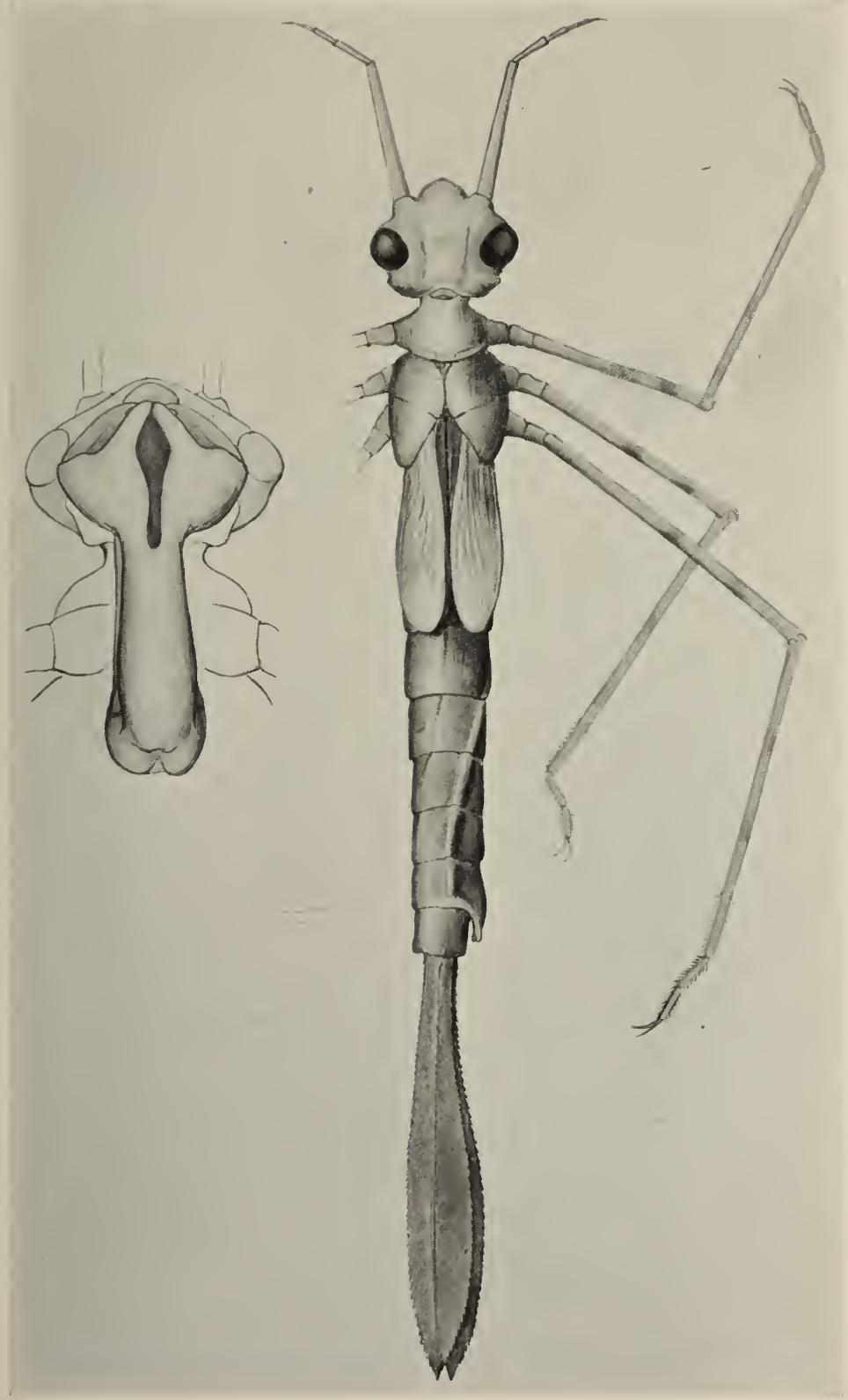

Plate I. AgRion aequabile

$5 \times$ Nat. Size 


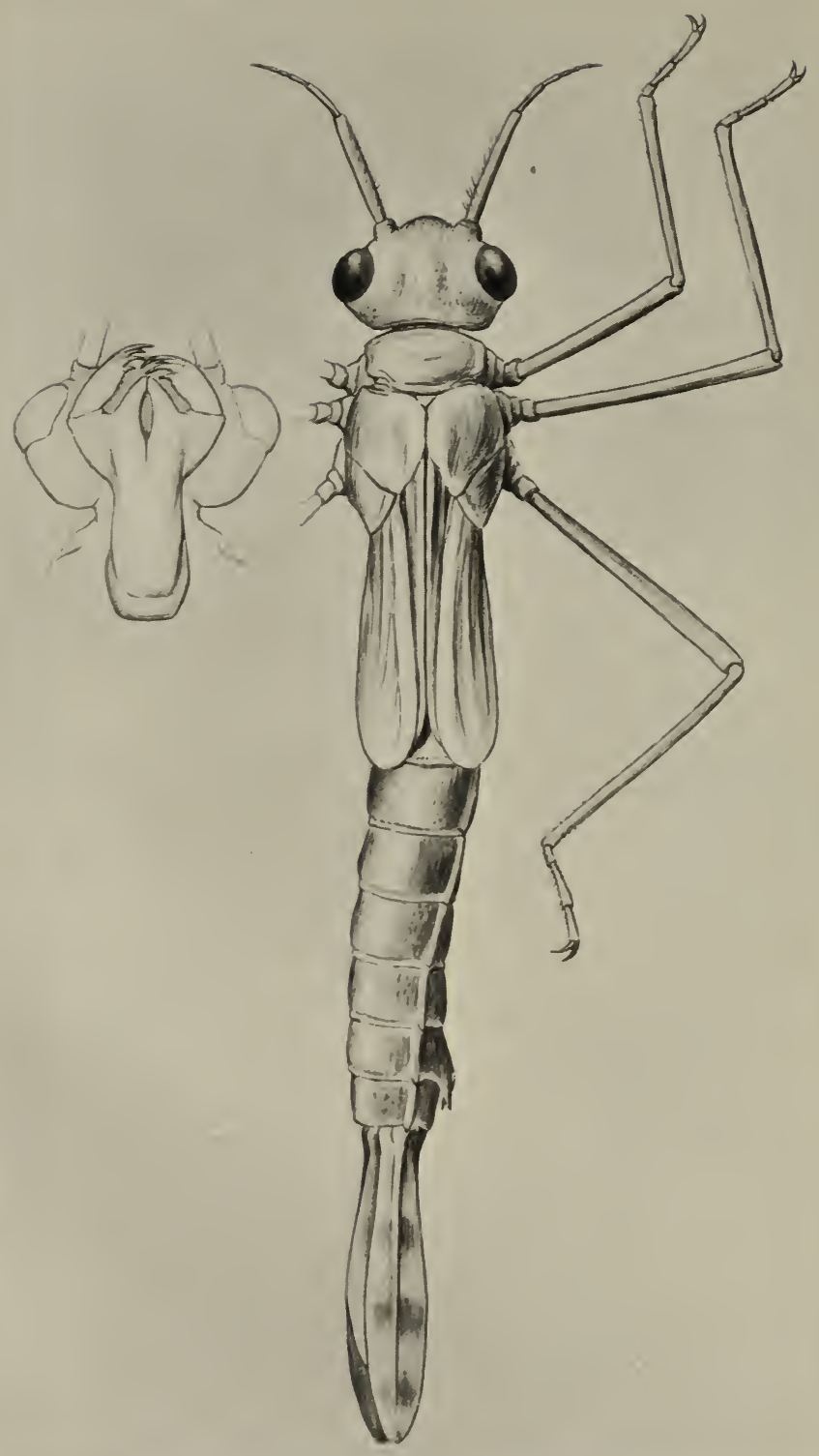

$5 \times$ Nat. Size

Plate II. HETAERINA AMERICANA 


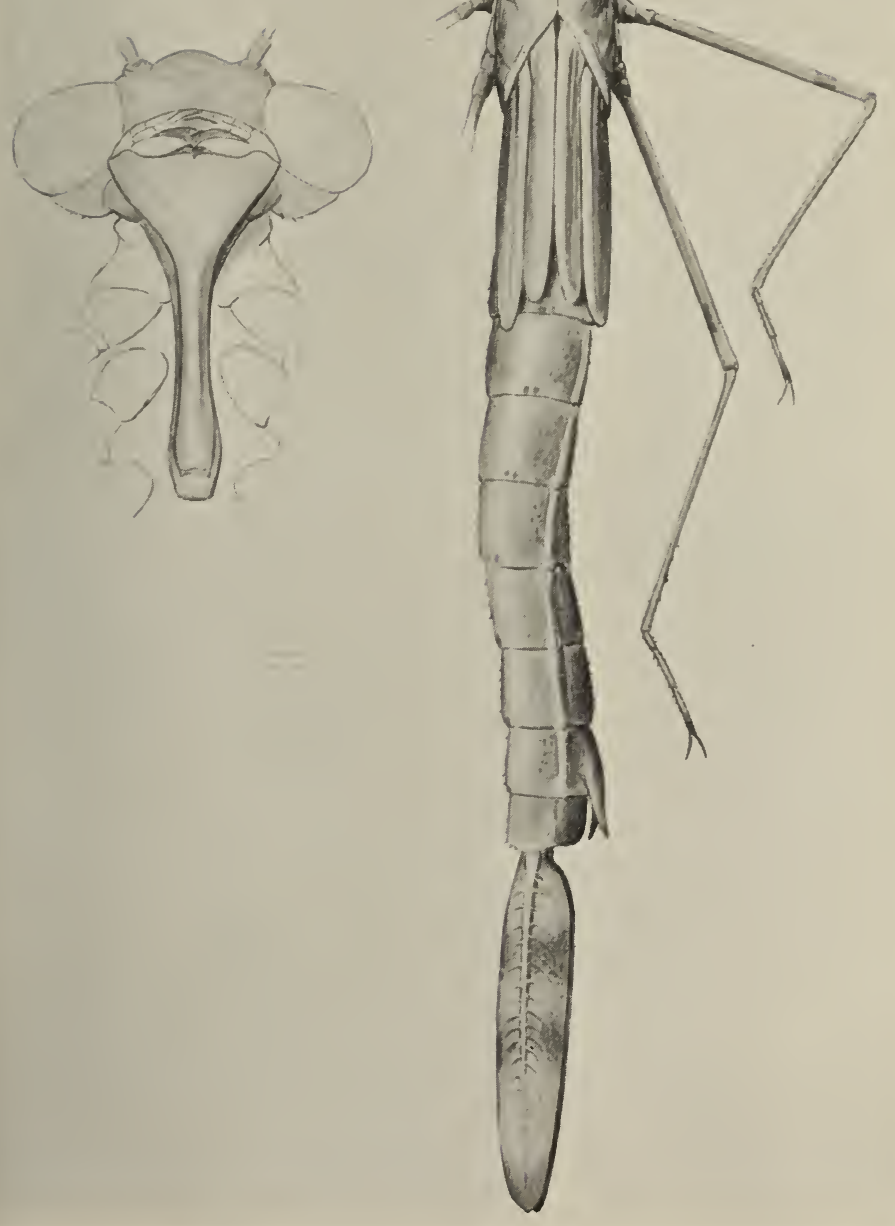

Plate III. Lestes ReCtangularis

$6 \times$ Nat. Size 


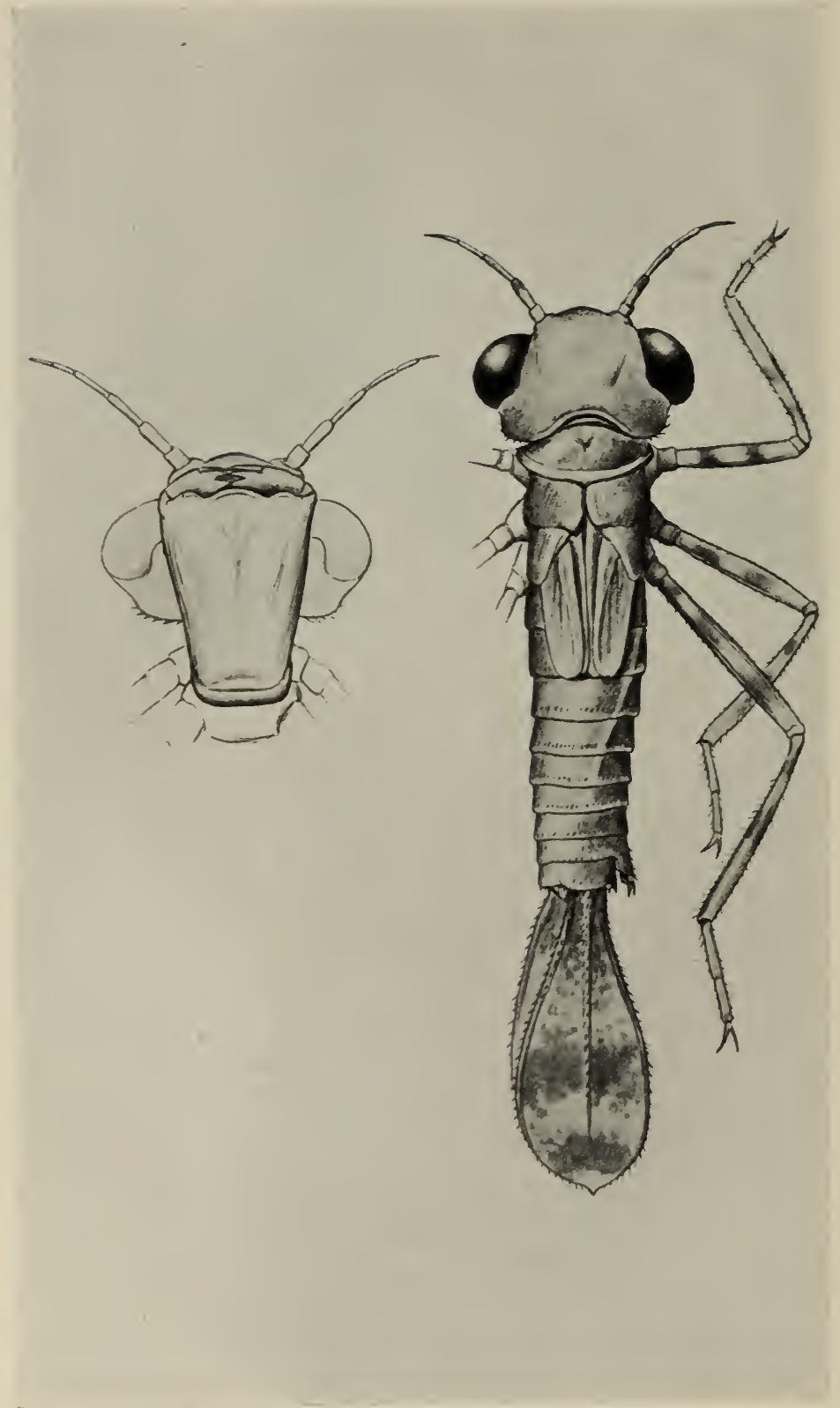

Plate IV. ARgia Violacea 

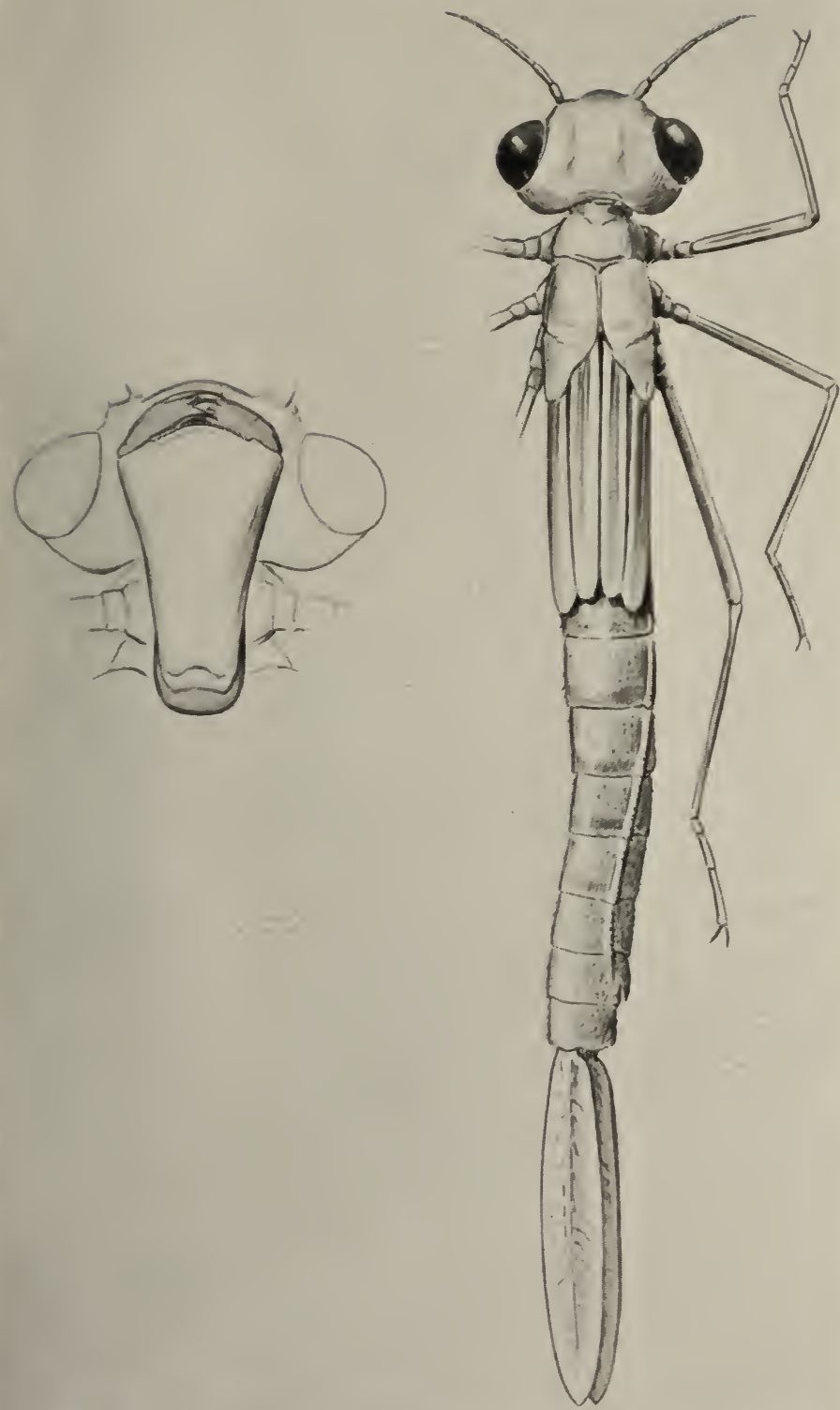

Plate V. Enallagma DURum

$6 \times$ Nat. Size 

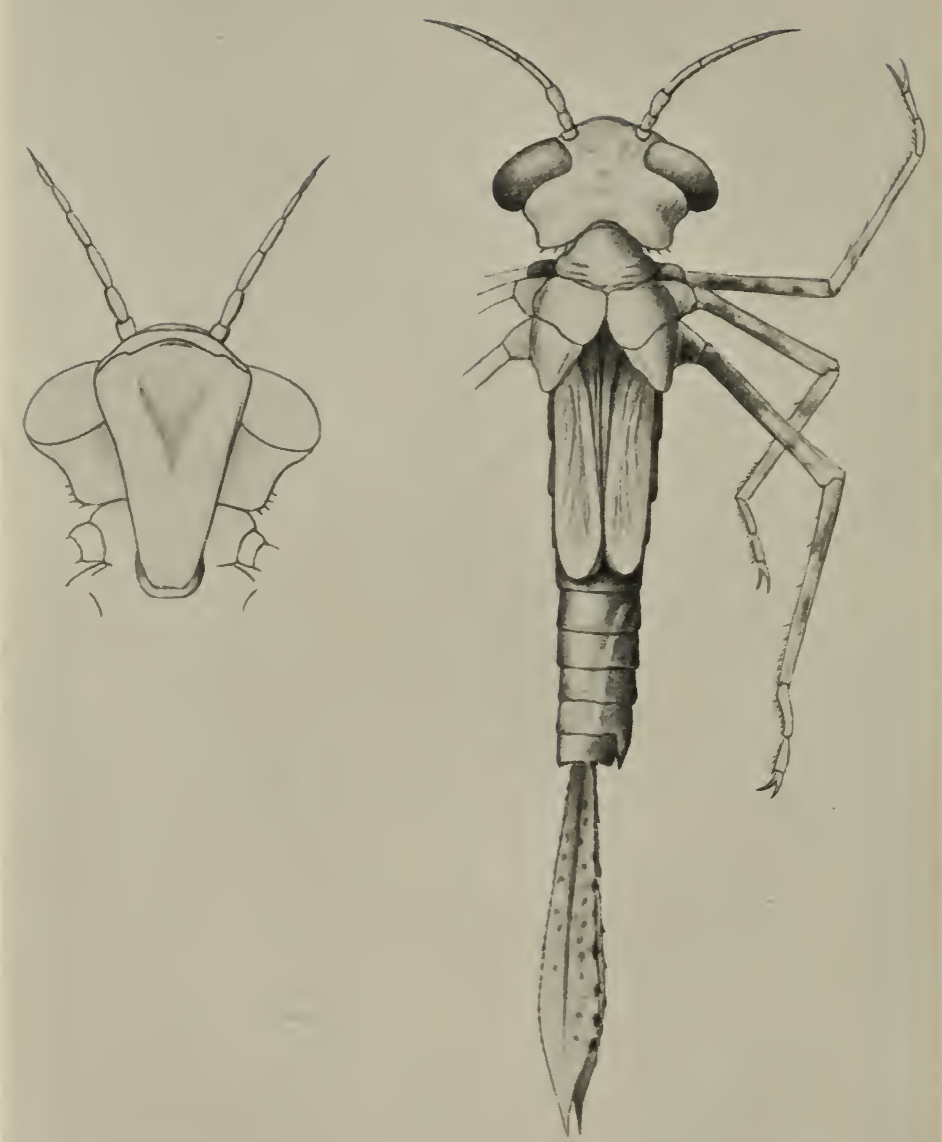

$7 \times$ Nat. Size 

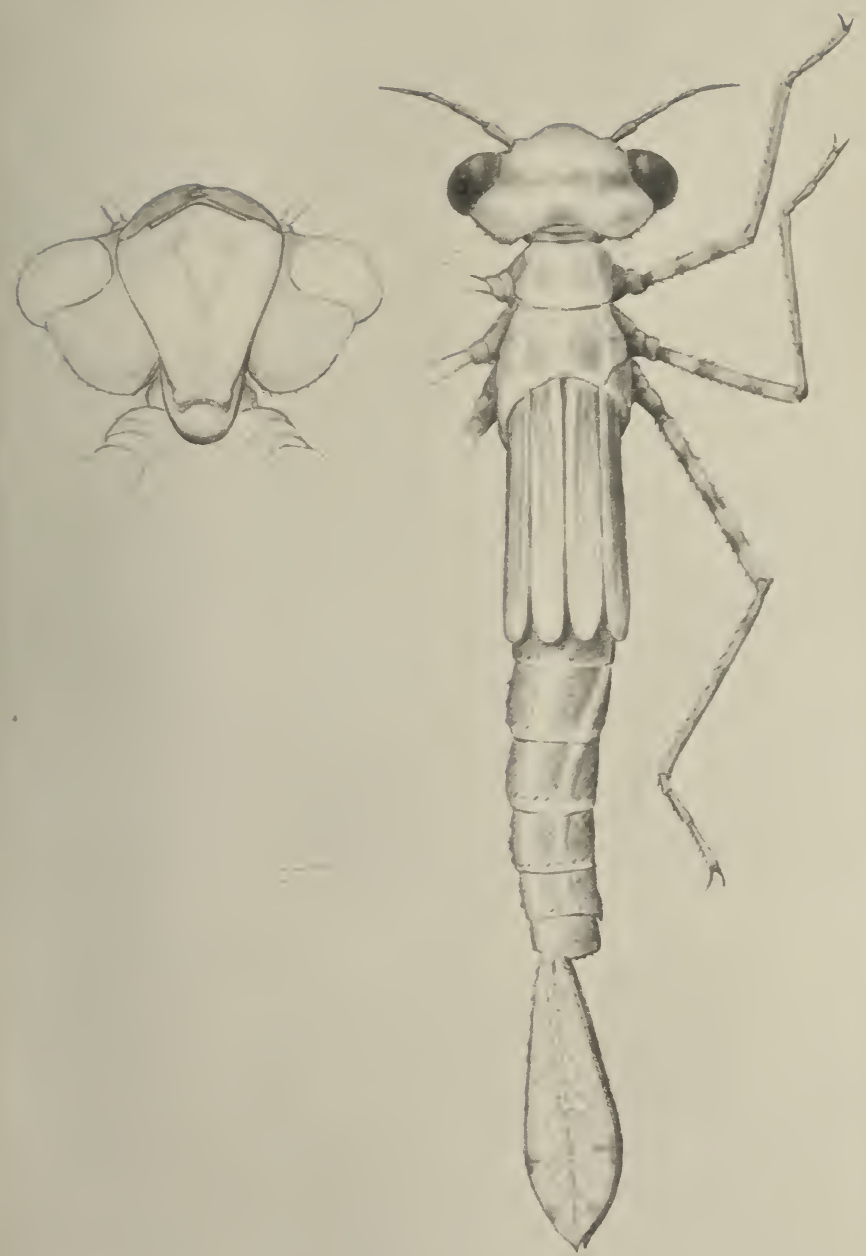

Plate VII. NEhalenNia IRENE

$9 \times$ Nat. Size 

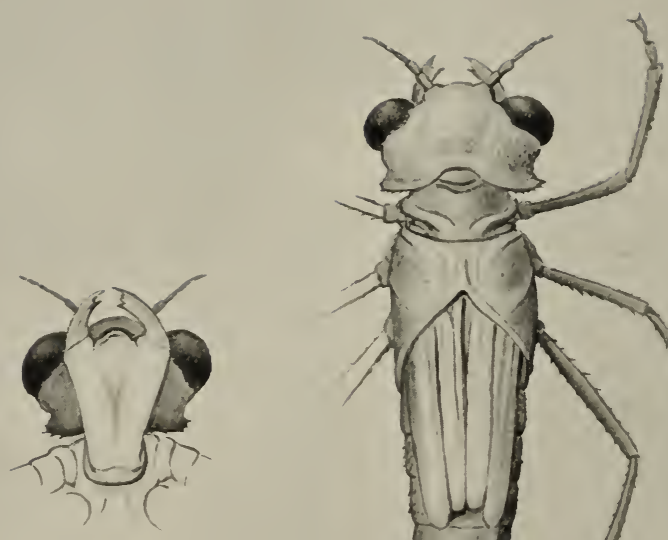

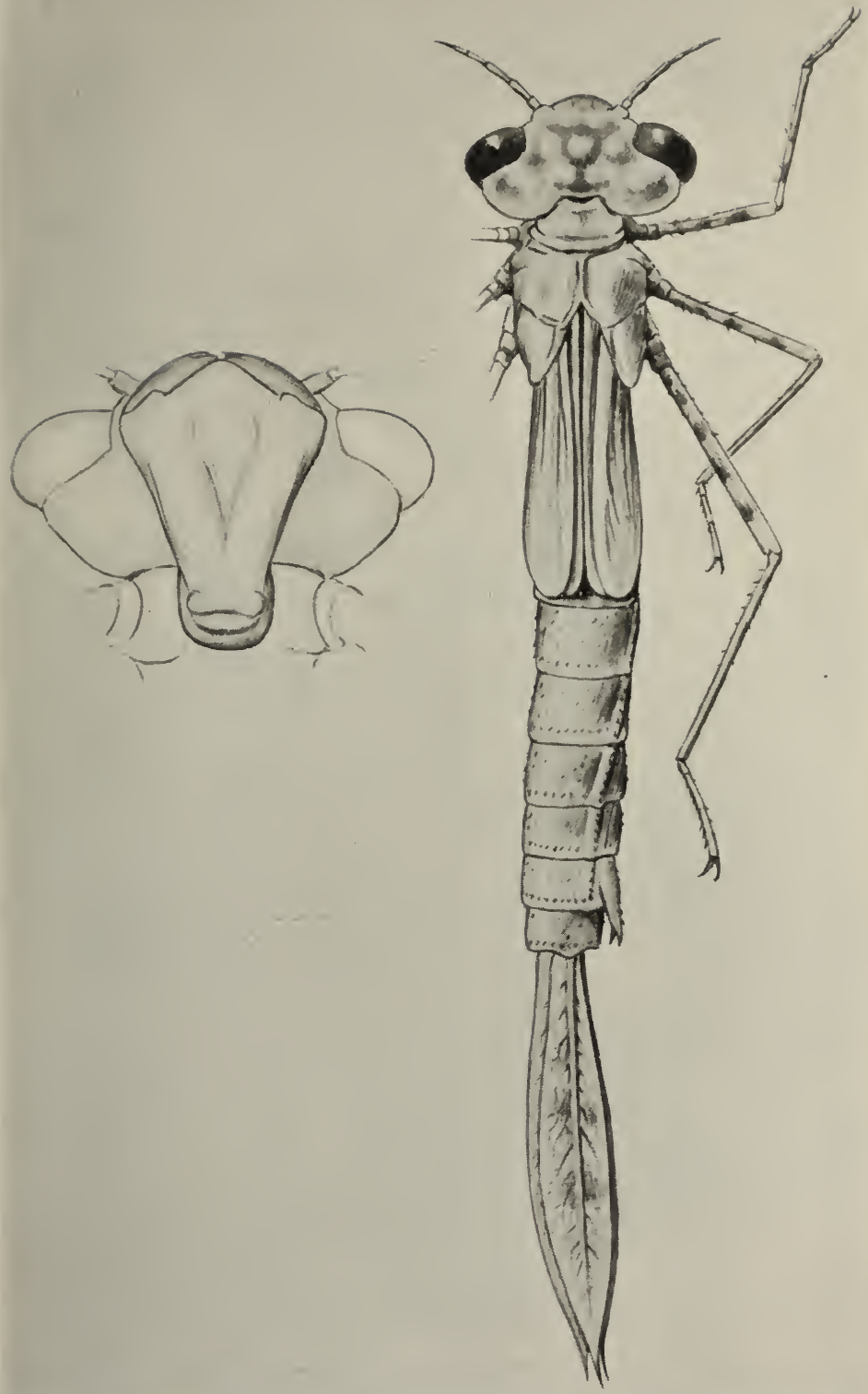

$9 \times$ Nat. Size

Plate IX. ISCHNURA VERTICALIS 


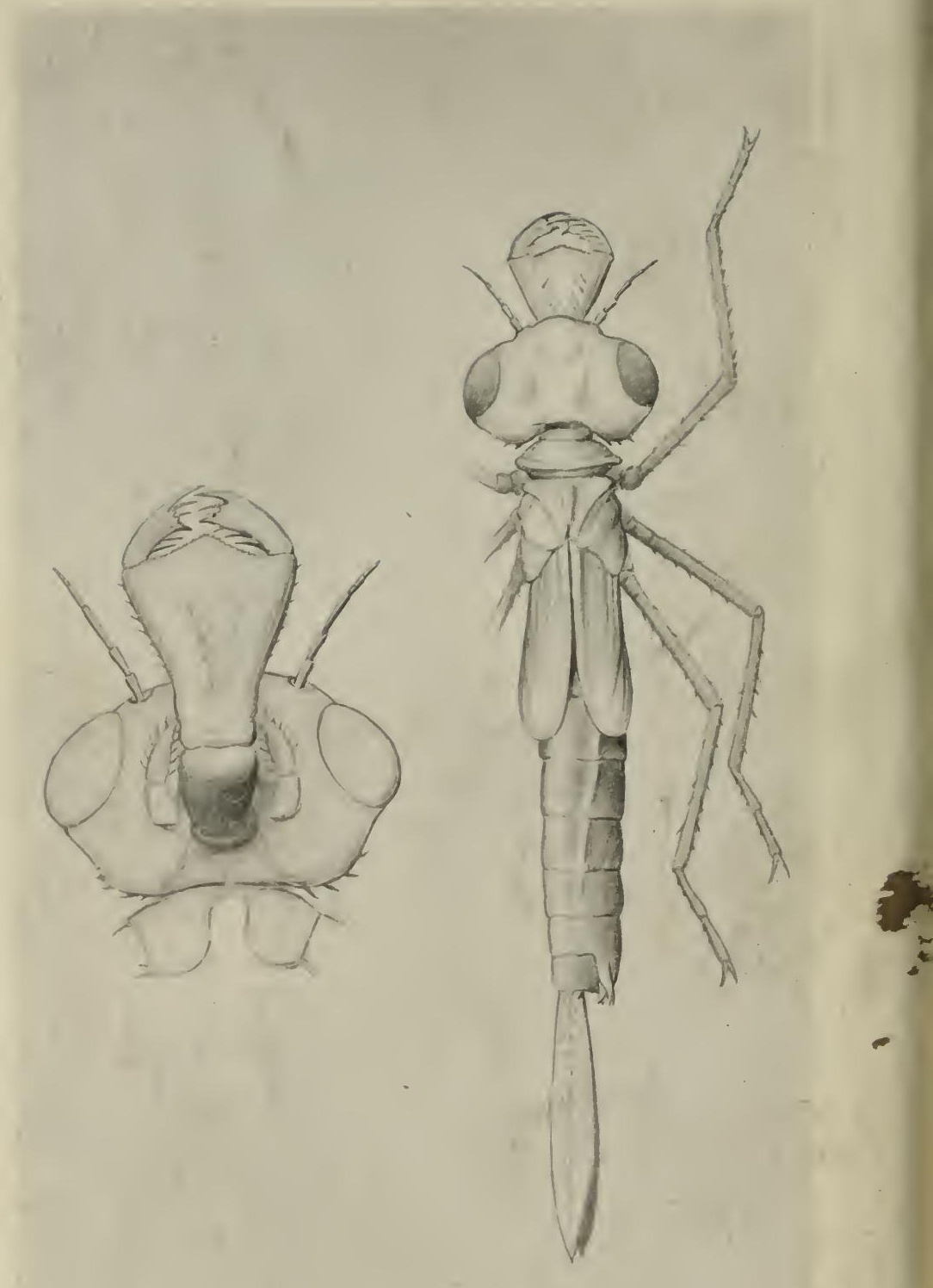

$8 \times$ Nat. Size

Plate X. ANOMALAGRION hastatum 


\title{
Memoir of the Thoreau Museum of Natural History : II Supplement to Manual of Odonata of New England
}

\author{
By R. HEBER HOWE, Jr.
}

March 15, 1921.

\section{Argia translata Hagen.}

Bull. Acad. Roy. Belg. (2) 20:410, 1865. Type locality:

"Venezuela, Porto-Cabello, par Appun."

One instance,- - ponds.

July 27, 1920.

Connecticut: Pistapaug pond, Wallingford (Garman).

b. Back of head black, abdomen banded with yellow.

158. Cordulegaster erroneus Hagen.

Bull. Acad. Roy. Belg (2) 46:688, 1878. Type locality: "Caroline du Nord aux environs de Morgantown."

June 8, 1911.

One instance, teneral.

Connecticut: Wallingford (Walden).

159. Gomphus amnicola Walsh.

Proc. Acad. Nat. Sci., Phila., 396, 1862. Type locality: "Illinois."

Dr. Muttkowski (ibid. 90) records this species from "Me.," but the record is untraceable.

Massachusetts: Amherst (Bromley).

160. Gomphus villosipes Selys.

Bull. Acad. Roy. Belg. 21:53, 1852. Type locality: "Etats-Unis."

Rare,-one instance.

June 15 to 30 .

Massachusetts: Amherst (Bromley).

Note: This species was included as from Massachusetts by Dr. P. P. Calvert (Tran. Amer. Ent. Soc. 20:245, Nat. Hist. 18:45, 1875), but was not included in Dr. Calvert's New England List, as Hagen later (Bull. Acad. Roy. Belg (2) 46:458, 1878) found the specimen from Natick to be $G$. furcifer.

c. Brown of hindwings reaching to nodus.

161. Tetragoneuria semiquaea (Burm.)

Handb. Ent. 2:858, 1839. Type locality: "Savannah."

Massachusetts: Dr. Muttkowski includes this species from Massachusetts in his Studies in Tetragoneuria (Odonata), Bull. Wis. Nat. Hist. Soc. 9:120, 1911.

162. Libellula exusta julia (Uhler)

Proc. Acad. Nat. Sci., Phila., 88, 1857. Type locality:

"Fort Steilacoom, W. Territory."

Mav 31.

Maine: Ogunquit (Blair-Smith).

New Hampshire: Jaffrey (Johnson). 
Massachusetts: Amherst (Bromley); Framingham (Warden); Salem (Walker); West Roxbury (B. S. N. H.) ; Plum Island (Morse).

Connecticut: New Haven (Walden).

* Teess entis f'y black.

163. Sympetrum atripes (Hagen)

U. S. Geol. Sur. Terr. Colo. 588, 1873. Type locality: "Yellowstone."

New Hampshire: Meredith, Sept. 9, 1919 (see Ent News, 28:1921).

** Sides of thorax with two yellow dots.

164. Sympetrum corruptum (Hagen.

Syn. Neur. No. Amer. 171, 1861. Type locality: "Pecos River, Western Texas," etc.

June 10 to September 10 .

Massachusetts: Chatham (Howe); Plum Island, Essex Co. (Morse).

Connecticut: New Haven (Walden).

Agrion amatum

July 19

Massachusetts: Petersham, 1,000 ft. (Wheeler).

Agrion aequabile

Maine: Monmouth (Frost).

Massachusetts: Middleton (Walker); Amherst (Bromley); Wareham (Bangs); Boston (Brues); Forest Hills (B. S. N. H.).

Connecticut: Mt. Carmel (Walden).

Agrion maculatum

Maine: Liberty (Frost).

New Hampshire: Fitzwilliain (Fenn).

Massachusetts: Petersham (Wheeler); West Roxbury (Taylor); Fram. ingham (Warden); Middleton (Walker); Boston (Brues); Am. herst (Bromley); Woburn (Anderson); Middleton, West Peabody (Morse); Forest Hills (Wheeler).

Connecticut: Goshen (Zappe).

Hetaerina americana

Massachusetts: Amherst (Taylor); Belchertown (M. C. Z.); Miller's Falls (Bromley).

Connecticut: Kent (Britton).

\section{Lestes eurinus}

Massachusetts: Amherst (Bromley); Woods Hole (Gray).

Connecticut: (C. A. E. S.).

Lestes congener

July 4.

Maine: Chamberlain lake (Cushınan).

New Hampshire: Fitzwilliam (Fenn).

Massachusetts: Stony brook, Forest Hills, Petersham (Wheeler); Heath (Warden); Hyannis (Howe).

Connecticut: Branford (Vierick); Westville (Britton); New Canaan (Garman)

Lestes unguiculatus

Massachusetts: Wellfleet (Johnson); Quincy (Anderson); Wenham, Peabody (Morse); Amherst (Bromley); Nantucket (Howe). 


\section{Lestes uncatus}

Maine: Ogunquit (Blair.Smith).

New Hampshire: Lake Sunapee (Johnstone).

Vermont: Bennington (Johnson).

Massachusetts: Woods Hole (Gray); Forest Hills (Brues); Amherst

(Bromley); Winchester (Atwater); Salem, Swampscott (Walker).

Rhode Island: Wickford (Johnson).

\section{Lestes disjunctus}

Maine: Glen House, Bar Harbor (Johnson); Ogunquit (Blair-Smith)

Vermont: Shoreham (Cushman).

Massachusetts: Hyannis, Harwich, Chatham, Waltham (Howe); Amherst (Bromley).

Connecticut: West Thompson (Vierick).

\section{Lestes forcipatus}

Massachusetts: Woods Hole (Gray); Amherst (Bromley); Phillipston (Wheeler).

Rhode Island: Block Island (Howe).

Connecticut: New Haven (Moore, Britton); Lake Whitney (Vierick).

\section{Lestes rectangularis}

Maine: Ogunouit (Blair-Smith).

New Hampshire: Sunapee (Johnstone).

Massachusetts: Tisbury (Cushman); Petersham (Wheeler); West Peabody, Wenham (Morse); West Roxbury (Taylor); Winchester (Atwater); Heath (Warden); Amherst (Bromley).

Connecticut: Branford (Winkley); New Haven (Buttrick).

\section{Lestes vigilax}

Meredith Neck (Calvert), should be under New Hampshire.

Maine: Liberty (Cushman).

Massachusetts: West Peabody (Morse); Hyannis, Wianno, Harwich (Howe); Cohasset (Bryant); Amherst (Bromley).

Connecticut: New Haven (Vierick).

Lestes inequalis

Maine: Ogunquit (Blair-Smith).

Massachusetts: Winchester (Atwater).

Connecticut: Litchfield, Stratford (Garman).

Argia moesta

Massachusetts: Petersham (Wheeler); Brewster (Howe).

Connecticut: Putnam (Vierick).

\section{Argia violacea}

September 26

Maine: Liberty (Cushman).

New Hampshire: Fitzwilliam (Gallagher).

Vermont: Shoreham (Cushman).

Massachusetts: Petersham (Wheeler); Cohasset (Bryant); Pocasset (Cushman): Danvers, Wenham (Morse); Ashburnham, Wianno (Howe); Winchester (Atwater); Carlisle (Taylor); Amherst (Bromley).

Connecticut: Orange (Britton); New Haven (Walden).

\section{Enallagma durum}

Massachusetts: Nantucket (Bromley); Wianno (Howe).

Connecticut: Woodmont, Branford (Buttrick). 
June 10 to July 18.

Maine: Bar Harbor (Johnson).

Vermont: South Hero (Cushman).

Massachusetts: Amherst (Bromley); Woods Hole (Gray).

Connecticut: New Haven (Walden).

\section{Enallagma hageni}

June 29 to August 10.

Maine: Belfast (Cushman); Monmouth (Frost).

New Hampshire: Sunapee (Johnstone).

Vermont: Coventry (Cushman).

Massachusetts: Danvers (Morse).

\section{Enallagma calverti}

Massachusetts: Framingham (Howe); Melrose (Anderson); Haverhill (Morse).

\section{Enallagma ebrium}

Maine: Ogunquit (Blair-Smith); Liberty (Cushman).

Vermont: Shoreham (Cushman).

Massachusetts: Salem (Walker); Amherst (Bromley); Winchester (Atwater); Lynn (Morse).

Connecticut: New Haven (Walden); West Thompson (Vierick).

Enallagma traviatum

Connecticut: Pistapaug pond, Wallingford (Garman).

\section{Enallagma aspersum}

Ju!y 9.

Massachusetts: Manomet (Cushman); Winchester (Atwater); Amherst (Bromley).

Connecticut: (C. A. E. S.)

Enallagma minusculum

Massachusetts: Winanno (Howe).

Enallagma doubledayi

June 28 to September 13.

Massachusetts: Hyannis, Chatham, Harwich (Howe); Woods Hole (Gray).

Enallagma carunculatum

Connecticut: (C. A. E. S.).

\section{Enallagma civile}

Salt marshes.

Massachusetts: Woods Hole, Tuckernuck island, West Tisbury (Cushman); Manomet (Johnson, Brooks); Wianno, Orleans, Brewster, Brookline (Howe); Woods Hole (Gray): Westfield, Amherst (Bromley); Rockport, West Peabody, Wenham (Morse); Horse Neck Beach (Holt); Tarpaulin Cove (Bryant).

Rhode Island: Block Island (Howe): Wickford (Atwater).

Connecticut: New Haven (Vierick); Whitneyville (Buttrick).

Enallagma geminatum

Massachusetts: West Peabody, West Andover (Morse).

Connecticut: Mt. Carmel (Moore); New Haven (Moore, Tierick).

Enallagma laterale

Rhode Island: Wickford (Johnson)

\section{Enallagma exsulans}

Massachusetts: Brewster (Howe).

Connecticut: Putnam (Vierick). 


\section{Enallagma signatum}

Massachusetts: Woods Hole (Gray); South Chatham, Orleans (Howe); Amherst (Bromley); Framingham (Warden); Peabody (Morse).

Connecticut: West 'Thompson (Vierick).

Enallagma pollutom should read Enallagma vesperum.. See Calvert, Trans. Amer. Ent. Soc. 45:381, 1919.

June 21.

Massachusetts: Manomet (Johnson); Cohasset (Bryant).

Connecticut: North Guilford, Litchfield, Mt. Carmel (Garman).

Nehalennia irene

June 20.

Maine: Belfast; Liberty (Cushman); Monmouth (Frost).

Massachusetts: Woods Hole (Cushman); Wenham (Morse); Amherst (Bromley).

Rhode Island: Bristol, Wickford (Johnson).

Connecticut: West Haven (Vierick).

Amphiagrion saucium

May 25 to July 20.

Vermont: Coventry (Cushman).

Massachusetts: Amherst (Bromley); Peabody, Danvers (Morse).

Rhode Island: Wickford (Johnson).

Connecticut: New Haven (Britton, Vierick, Winkley).

Chromagrion conditum

New Hampshire: Jaffrey (Johnson).

Connecticut: (C. A. E. S.).

\section{Ischnura vertjcalis}

Salt marshes.

Maine: Liberty. Belfast (Cushman).

New Hampshire: Sunapee (Johnstone).

Verinont: Derby (Cushman); Bennington, Manchester (Johnson).

Massachusetts: Horse Neck Beach (Holt); Woods Hole, Nantucket, Tuckernuck, Nomanesset Island (Cushman); Wellfleet, Edgartown (Johnson); Tarpaulin Cove (Bryant); Melrose (Anderson); Brewster, Orleans, Ashburnham, South Chatham, Hyannis, Wianno, Block Island, Harwich (Howe); Salem (Walker); Woods Hole (Gray); Amherst (Bromley); Lynnfield, Rockport (Morse); Winchester (Atwater); Amherst, West Roxbury (Taylor).

Connecticut: Portland (Walden); West Thompson, Lake Whitney (Vierick); Poquonock (Britton); Mt. Carmel (Moore); South Manchester (Sturgis).

Ischnura posita

Massachusetts: Cohasset (Bryant); Tisbury (Cushman).

Rhode Island: Wickford (Johnson).

Connecticut: Winnepauk (Johnson).

Corãulegaster diastatops

Vermont: Benningion (Johnson).

Massachusetts: Amherst (Bromley).

a. Black of head brown, abdomen spotted with yellow.

\section{Cordulegaster maculatus}

Maine: Monmouth (Frost).

Massachusetts: Amherst (Bromley); Framingham (Warden).

Connecticut: Rainbow (Kirk).

"'Pepperill (Hawker)", should read, Pepperill (Parker). 
Cordulegaster obliquus

Connecticut. Westrille (Walden).

Insert b. Back of head black, abdomen banded with yellow.

Progomphus obscurus

August 21.

Massachusetts: Brewster, South Chatham, Wianno (Howe).

Hagenius brevistylus

June 15.

Massachusetts: Essex Co. (Morse); Amherst (Bromley).

Ophiogomphus mainensis

Massachusetts: Dr. Muttkowski includes "Mass." in his list of Odonata of North America-the record cannot be traced.

Ophiegomphus johannus

Connecticut: (C. A. E. S.).

Ophiogomphus rupinsulensis

June 14.

Massachusetts: Great Barrington (Johnson).

Connecticut: (C. A. E. S.).

Ophiogomphus aspersus

Massachusetts: Amherst (Bromley).

Gomplus albistylus

Connecticut: (C. A. E. S.).

Gomphus parvulus

Maine: Monmouth (Frost).

Massachusetts: Chester, Great Barrington (Johnson).

Gomphus brevis

Massachusetts: Mt. Ton (Needham); Amherst (Bronley).

Connecticut: (C. A. E. S.).

Gomphus exilis

New Hampshire: Squan lake (Allen); Fitzwilliam (Howe).

Massachusetts: Framingham (Warden, Frost); Manomet (Johnson); Anherst (Bromley); Orleans. Chatham (Howe); West Peabody (Morse); Medford (Anderson); Forest Hills (Brues); Winchester (Atwater).

Connecticut: West Thompson (Vierick); New Haven. Westrille, Mt. Carmel (Walden).

Gomphus lividus

Massachusetts: Amherst (Bromley).

Connecticut: (C. A. E. S.).

Gomphus ventricosus

June 10.

Massachusetts: Amherst (Bromles).

Gomphus adelphus

Massachusetts: Cambridge? (Hagen).

Gomphus borealis

New Hamphire: Jaffrey (Johnson).

Connecticut: (C. A. E. S.)

Gomphus spicatus.

Maine: Bar fIarbor (Johnson).

Vermont: Manchester (Johnson).

Massachusetts: Amherst (Bromley).

Rhode Island: Washington (Johnson).

Connecticut: (C. A. E. S.). 


\section{Gomphus furcifer.}

Massachusetts: Essex Co. (Morse).

\section{Gomphus scudderi}

Maine: Orouo (Harvey).

Dromogomphus spinosus

June 12.

New Hampshire: Fitzwilliam (Howe).

Iassachusetts: Chatham (Howe); Amherst (Bromley).

Connecticut: (C. A. E. S.).

Boyeria vinosa

Septemiber 26.

Massachusetts: Petersham (Wheeler); Framingham (Warden); Brookline (Shurtleff); West Townsend (Howe); Amherst (Bromley).

\section{Boycria grafiana}

August 7, 1920.

New Hampshire: Sunapee (Johnstone).

Basiaeschna janata

August 13.

Massachusetts: Amherst (Bromley); Framingham (Howe); Carver (B. S. N. H.).

Connecticut: (C. A. E. S.).

\section{Gomphaeschna furcillata}

July 28.

Massachusetts: Salem (Walker); West Peabody (Morse).

Connecticut: Westville (Britton); Lyme (Zappe); New Haven (Lowry); North Branford (Walden).

\section{Anax junius}

Maine: Ogunquit (Blair-Smith).

Massachusetts: Amherst (Bromley); Winchester (Atwater); Woods Hole (Gray); Winchendon (Johnson); Peabody (Morse); Hyannis (Howe).

Rhode Tslard: Block Island (Howe).

Connecticut: New Haven (Britton); Whitneyville (Buttrick); Milford (Vierick).

\section{Anax longipes}

Note: Hagen's record (Ent. Mo. Mag. 20:169-170, 1884) was of two males and one female that were taken flying about the "tops of trees on [a] hill."

Aeshna umbrosa

November 1.

Maine: Bar Harbor, North-east Harbor (Johnson); Bethel (Lamb).

New Hampshire: Sunapee (Johnstone); Meredith (Wheeler); Jaffrey, Fitzwilliam (Howe).

Massachusetts: Princeton (Johnson); Heath (Warden); Amherst (Bromley); Blue Hill (Taylor); Watertown, Arlington (Anderson); Ashby, Ashburnham (Howe); Westboro (Fay); Wenham (Morse).

Connecticut: (C. A. E. S.).

\section{Aeshna interrupta}

September 18.

Massachusetts: Wenham (Morse). 


\section{Aeshna clepsydra}

Maine: Bar Harbor (Johnson); Liberty (Cushman).

Massachusetts: Amherst (Bromley); Wianno, Hyannis, Brewster (Howe); Brookline (Shurtleff).

Connecticut: (C. A. E. S.).

\section{Aeshna mutata}

June 5, 1902

\section{Aeshna verticalis}

July 17.

New Hampshire: Sunapee (Johnstone).

Massachusetts: Forest Hills (Wheeler); Arlington (Anderson); Salem (Morse); Anlherst (Bromley); Chatham (Howe).

Connecticut: Salisbury, New Haven (Britton); Scotland (Walden).

\section{Aeshna tuberculifera}

August 1 to September 23

Massachusetts: South Orleans, Hyannis, Chatham (Howe;) Wrentham (Frost); Heath (Warden).

Connecticut: (C. A. E. S.).

\section{Aeshna canadensis}

Maine: Bar Harbor (Johnson).

New Hampshire: Sunapee (Johnstone); Fitzwilliam (Gallagher).

Massachusetts: Sonth Orleans, Ashburnham (Howe); Petersham (Wheeler); Heath (Warden); Amherst (Bromley).

Connecticut: (C. A. E. S.).

Aeshna constricta

Massachusetts: Amherst (Bromley); Essex Co. (Morse).

Nasiaeschna pentacantha

June 14.

Massachusetts: Wellesley (Needham); Concord (Howe); Dedham (Clark); (see Psyche 27:154, 155, 1920).

\section{Epiaeschna heros}

September 17.

New Hampshire: Mt. Monadnock (Johnson).

Massachusetts: Woods Hole (Gray); Marblehead, Swampscott (Walker); Salem, Marblehead (Morse); Amherst (Bromley).

Connecticut: Lyme (Zappe); New Haven (Lowry); North Branford (Walien).

\section{Didymops transpersa}

Maine: Capens (Johnson); Monmouth (Frost).

New Hampshire: Squam lake (Allen); Jaffrey (Johnson).

Massachusetts: Rutland (Johnson); Melrose (Anderson); Framing. ham (Howe); Amherst (Bromley); Salem (Morse). Connecticut: Mt. Carmel (Walden).

\section{Macromia illinoiensis}

June 16.

New Hampshire: Fitzwilliam (Fenn).

Massachusetts: Amherst (Bromley); Wianno (Howe).

\section{Williamsonia lintneri}

Massachusetts: Framingham (Frost); Middleton (Walker); Hopkin. ton (Frost).

\section{Dorocordulia lepida}

Massachusetts: Sharon (Johnson); West Peabody (Morse).

Rhode Island: Washington (Johnson).

Connecticut: (C. A. E. S.). 


\section{Dorocordulia libera}

New Hampshire: Mt. Monadnock (Johnson).

Massachusetts: Amherst (Bromley).

Connecticut: (C. A. E. S.).

Epicordulia princeps

Massachusetts. Brewster, Wianno, Hyannis (Howe); Woods Hole (Gray).

Connecticut: (C. A. E. S.).

Helocordulia uhleri

Massachusetts Amherst (Bromley).

Connecticut: (C. A. E. S.).

Somatochlora cingulata

Massachusetts: Dr. Muttkowski includes Massachusetts in his Odonata of North America, but the record seems untraceable.

Somatochlora elongata

August 21.

New Hampshire: Sumapee (Johnstone).

Somatochlora linearis

July 2.

Connecticut: Orange (Zaype).

Somatochlora williamsoni

August 8.

Now Hampshire: Sunapee (Johnstone).

Somatochlora kelnedyi

Massachusetts: Middleton (Walker).

Somatochlora minor

Connecticu:: (C. A. E. S.).

Somatochlora tenebrosa

Connecticut: (C. A. E. S.)

Cordulia shurtleffi

New Hampshire. Jaffrey (Johnson).

Connecticut: (C. A. H. S.).

Tetragoneuria cynosura

July 12

Mascrchusetts: Framingham (Warden); Chatham, Boston (Howe); Amherst (Bromley); Cambridge (Lamb); Arlington (Anderson); Woods Hole (Gray).

Connecticut: West Thompson (Vierek); Mt. Carmel, New Haven (Walden).

Tetragoneuria c. simulans

Maine: Monınouth (Frost); South-west Harbor (Johnson).

New Hampshire: Squam lake (Allen).

Vermont: Bennington (Johnson).

Massachusetts: Woods Hole (Gray); Framingham (Howe); Melrose (Anderson); Danvers (Morse).

Connecticut: (C. A. E. S.).

under A.

c. Brown of hindwings reaching to nodus.

Tetragoneuria spinigera

New Hampshire: Fitzwilliam (Howe); Jaffrey (Johnson).

Vormont: Manchester (Johnson).

Massachusetts: Littleton (Howe); Winchester (Atwater).

Connecticut: Goshen (Zappe). 
Tetragoneuria canis

May 31.

Maine: Manchester (Wadsworth); Dr. Muttkowski gives this record ibid. 132.

Massachusetts: Concord (Howe). (Psyche $27: 155,1920$.

Connecticut: (C. A. F. S.).

Libellula auripennis

Septembe" 13.

Massachusetts: Harwich, Hyannis, Chatham (Howe); Woods Hole (Gray).

Connecticut: (C. A. E. S.).

Libellula incesta

Massachusetts: Amherst (Bromley); Harwich, Wianno, South Chatham (Howe); Woods Hole (Gray); West Peabody (Morse).

Libellula vibrans

July 7.

Maine: Dr. Muttkowski includes this species from Maine (ibid. 141), but the recorri is untraceable and very doubtful.

Massachusetts: Woods Hole (Gray).

Connecticut: East Haven (Walden).

\section{Libellula flavida}

The Concord record must be expunged, as the specimen determined by Mr. Williamson has since been pronounced by Dr. Calvert as L. cyanea, though showing some misleading characters. The Wareham record is therefore also referable to $L$. cyanea. As the author lad not listed definitely $L$. favida as a New England species, because certain discrepancies were evident if the Concord species were true $L$. flavida-he wishes now to withdraw the last sentence in the Note on page 70. L. flavida, Ramb., is apparently sufficieritly distinct to be recognized.

Libellula cyanea

Massachusetts: Amherst (Bromley); Harwich (Howe); Woods Hole Gray); Danvers (Morse).

Connecticut: New Haven (Moore).

\section{Libellula pxusta}

New Hampshire: Squam lake (Allen).

Massachusetts: Framingham (Frost); Winchendon (Johnson); Woods Hol، (Gray); Amherst (Bromley); Chatham (Howe); West Peabody (Morse).

Libellula luctunsa

June 3.

Massachusetts: Amherst (Bromley); Danvers, Lynn, West Peabody, Boxford (Morse).

Rhode Island: Block Island (Howe).

Connecticut: New Haven (Vierick); Southington (Walden).

Libellula quadrimaculata

Maine: South-west Harbor (Johnson).

New Hampshire: Hanover (Britton).

Massachusetts: Amherst (Bromley); Swampscott (Walker); Woods Hole (Gray); Plum Island (Morse).

Libellula semifasciata

Massachusetts: Amherst (Bromley); Woods Hole (Gray); Salem, West Peabody (Morse).

Connecticut: New Haven (Walden). 
Libellula pulchella

Maine: Ogunquit (Blair-Snith).

Massachusetts: Harwich, Wianno, South Chatham, Hyannis (Howe); Winchester (Atwater); Amherst (Bromley); Salem (Walker); Forest Hills (Henshaw, Wheeler); Beverly (B. S. N. H.); Medford, Watertown (Anderson); Woods Hole (Gray); West Peabody, Danvers (Morse).

Rhode Island: Block Island (Howe).

Connecticut: Durham (Zappe); West Haven (Britton); Branford (Vierick); New Haven (Walden); Whitneyville (Buttrick).

Plathemis lydia

May 10 to September 23.

Maine: Bar Harbor (Johnson).

Massachusetts: Andover (B. S. N. H.); Cambridge, Waltham (Howe); Woods Hole (Gray); Winchester (Atwater); Amherst (Bromley); Forest Hills (Brues); Great Barrington (Johnson); Lynnfield, West Yeabody, Wenham, Salem, Danvers (Morse).

Connecticut: Branford (Winkley); East Haven (Walden).

Perithemis domitia tenera

June 8 .

Massachusetts: Winchester (Atwater); South Chatham (Howe); East Wareham, Amherst (Bromley); Woods Hole (Gray); West Peabody (Morse); Amherst (Taylor); Auburndale (Johnson).

Rhode Island: Block Island (Howe).

Connecticut: West Thompson (Vierick); New Haven (Britton); Whitneyville (Buttrick).

Nannothemis bella

June 7 .

Massachusetts: South Chatham (Howe); Amherst (Bromley).

Rhode Island: Wickford (Johnson).

Connecticut: New Haven (Walden).

Erythrodiplax berenice

August 28

Massachusetts: Cambridge (Bromley); Woods Hole (Cushman, Gray); Boston, Revere (B. S. N. H.); Plum Island (Morse).

Connecticut: Stratford (Britton); Stonington (Hyslop); Short Beach (Buttrick).

Erythemis simplicicollis

September 13.

Massachusetts: Harwich, Wianno, Hyannis, South Chatham, Chatham (Howe); Amherst (Bromley); Woods Hole (Gray); Boston (B. S. N. H.) ; West Peabody (Morse).

Rhode Island: Block Island (Howe).

Connecticut: East Haven (Buttrick).

Pachydiplax longipennis

Massachusetts: Woods Hole (Gray); Chatham, Harwich, Brewster (Howe); West Peabody (Morse).

Rhode Islani: Block Island (Howe).

Connecticut: New Haven (Walden).

Sympetrum rubicundulum

Maine: Roque Bluffs, South West Harbor, Ashland Junction, Mt. Desert (Johnson); Chamberlain Lake (Cushman); Ogunquit (Blair-Smith).

New Hampshire: Fitzwilliam (Howe); Sunapee (Johnstone): Monadnock, Glen House (Johnson). 
Massachusetts: Woods Hole (Gray); Danvers, West Peabody, Salem (Morse); Harwich, Waltham, Brookline, Brewster, Wianno, South Chatham, Hyannis (Howe); Petersham, Blue Hill, Stony Brook, Forest Hills (Wheeler); Salem (Walker); Woods Hole (Cushman); Weston (Paine); Winchester (Atwater); Amherst (Bromley); Weston, Sunderland, West Roxbury, Carlisle (Taylor); Martha's Vineyard, Brookline, Wellfleet, Auburndale (Johnson).

Rhode Island: Block Island (Howe).

Connecticut: South Manchester (Sturgis); Westville, Buttrick (Britton); New Haven (Zappe, Noore, Walden, Vierick); Branford. Westbrook (Vierick); Short Beach (Buttrick); Stonington (Hyslop).

* Sides of thorax unmarlied.

**Sides of thorax with two yellow dots.

Put after " 1 . Wings over $25 \mathrm{~mm}$. long."

Sympetrum obtrusum

Maine: Fryeburg (Harvey) record expunged by Williamson.

Massachusetts: Blue Hills (Wheeler); Woods Hole (Gray).

\section{Sympetrum semicinctum}

June 29.

Massachusetts: Heath (Warden); Winchester (Atwater); Amherst (Broinley); Plum Island (Morse).

Connecticut: Chapinville (Britton).

after "b. Wings with front margin yellow, $27 \mathrm{~mm}$. long." read

* Legs marked with yellow.

**Legs entirely black.

Sympetrum costiferum

Massachusetts: Harwich, Wianno, Chatham (Howe); Manomet (Brooks); Nantucket (Cushman).

Connecticut: (C. A. E. S.).

Sympetrum vicinum

Maine: Bar Harbor (Johnson).

New Hampshire: Nt. Monadnock (Johnson); Fitzwilliam, Rindge, Jaffrey (Howe): Meredith (Wheeler).

Massachusetts: West Roxbury, Cambridge, Boston (B. S. N. H.); Danvers (Morse); Salem (Walker); Tisbury (Cushman); Manomet (Brooks); Petersham, Stony Brook, Blue Hills (Wheeler); Petersham (Taylor); Cambridge, Ashburnham, Brewster, South Chatham. Chatham (Howe); Heath (Warden); Winchester (At. water); Amherst (Bromley).

Connecticut: Stonington (Hyslop); New Haven (Vierick).

\section{Leucorrhinia intacta}

Maine: Monmouth, Wales (Frost).

New Hampshire: Sunapee (Johnstone); Jaffrey (Johnsen).

Massachusetts: Woods Hole (Gray); Amherst (Bromley); Boston (Henshaw); Blue Hills (B. S. N. H.); Salem (Walker); Danvers. Lynn (Morse); Medford (Anderson); Great Barrington (Johnson).

Rhode Island: Bristol, Washington (Johnson).

Connecticut: Lyme, Mt. Carmel (Walden); New Haven, Lake Whitney (Vierick).

\section{Leucorrhinia gracilis}

Connecticut: (C. A. E. S.). 


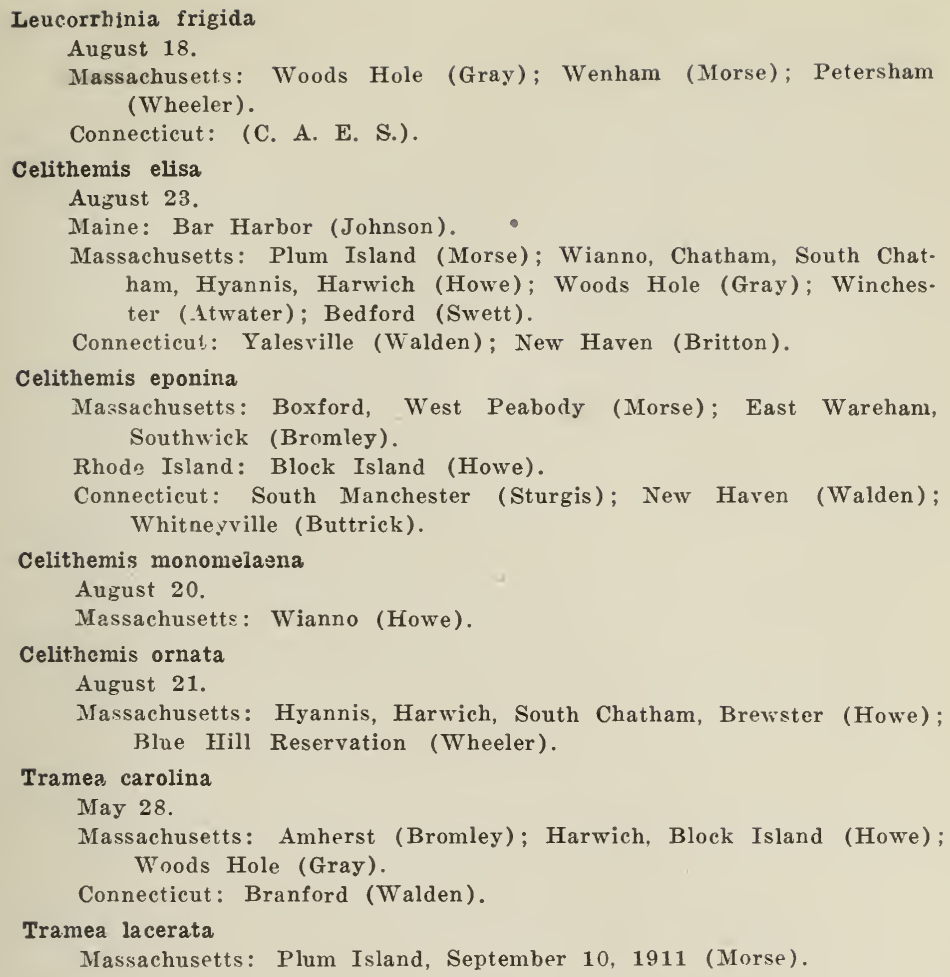

\section{BIBLIOGRAPHY}

1880: Torrey, Bradford. Migration of Dragon Flies, Amer. Nat. 14:132-133. 1881: Torrey, Bradford. Second Flight of Dragonflise, Amer. Nat. 14:594. 1891: Bullock, David Jayne. Notes on Mt. Desert Dragonflies, Ent. News, 2:93-94.

1892: Hitchings, E. F. List of Dragonflies taken at Lake Quinsigamond, Worcester, Mass. Ent. News, 3:39.

1894: Calvert, Philip P. Data on the Distribution of Dragonflies (Odonata)-I. Ent. News, 5:242-244.

1914: Woodruff, Lewis B. The Nymph of Ophiogomphus johannus Needham. Journ. N. Y. Ent. Soc. 22:61-63.

1914: Williamson, E. B. Sympetrum obtrusum and costiferum (Odonata) in Maine. Ent. News, 25:456.

1915: Calvert, P. P. Odonata. Ent. News, 26:238-239.

1916: Osburn, Raymond C. A Migratory Flight of Dragonflies. Journ. N. Y. Ent. Soc. 24:90-92.

1918: Calvert, P. P. Odonata. Ent. News. 24:37-38.

1920: Britton, Wilton Everett. Check-List of the Insects of Connecticut, Bull 31. State Geol. and Nat. Hist. Surv., Odonata, 33-37. App. 354. 
In this latter work, the Connecticut record for Aeschna juncea, page 35 , and for Somatochlora elongata var. minor, page 36 , have proved to be errors, and should be expunged.

The author wishes here to thank his students, Messrs. C. W. Atwater, R. McP. Blair-Smith, C. C. Johnstone, J. O. D. Rosecrantz, and C. T. R. Bates, for much collecting in the field; Drs. W. E. Britton and Philip Garman, and Messrs. C. S. Anderson, F. H. Walker and C. A. Frost, for material supplied, beside many of those named before, who have continued to give raluable aid. 



\section{Date Due}

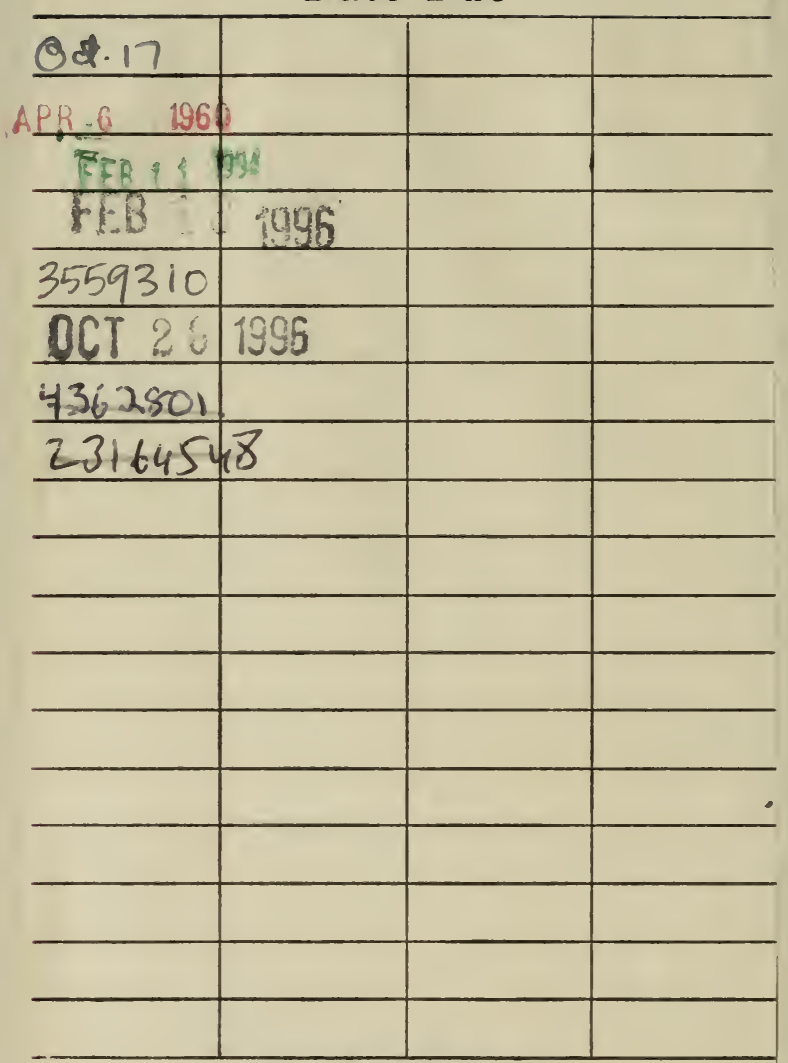

Library Bureau Cat. No. 1137 


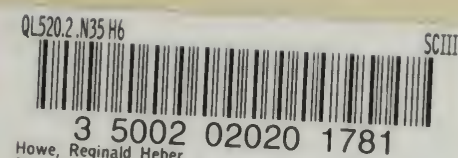

Howe, Reginald Heber 020201781

Manual of the Odonata of New England /

ZOOLOGY LISRARY

QL

520.2

N35Hó

255214 
